\title{
Renormalizable enhanced tensor field theory: The quartic melonic case
}

\author{
Joseph Ben Geloun ${ }^{1,2, a)}$ and Reiko Toriumi, ${ }^{3, b)}$ \\ ${ }^{1}$ Laboratoire d'Informatique de Paris Nord UMR CNRS 7030 Université Paris 13, \\ 99, Avenue J.-B. Clement, 93430 Villetaneuse, France \\ ${ }^{2}$ International Chair in Mathematical Physics and Applications (ICMPA-UNESCO Chair), \\ University of Abomey-Calavi, 072 B.P. 50, Cotonou, Republic of Benin \\ ${ }^{3}$ Institute for Mathematics, Astrophysics, and Particle Physics, Radboud University, \\ Heyendaalseweg 135, 6525 AJ, Nijmegen, The Netherlands
}

(Received 15 January 2018; accepted 24 October 2018; published online 28 November 2018)

\begin{abstract}
Tensor field theory is the quantum field theoretic counterpart of tensor models and enhanced tensor field theory enlarges this theory space to accommodate "enhanced tensor interactions." These interactions were introduced to explore new large $N$ limits and to probe different phases for tensor models. We undertake the multi-scale renormalization analysis for two types of enhanced theories with rank $d$ tensor fields $\phi:\left(U(1)^{D}\right)^{d} \rightarrow \mathbb{C}$ and with the so-called quartic "melonic" interactions of the form $p^{2 a} \phi^{4}$ reminiscent of derivative couplings expressed in momentum space. Scrutinizing the degree of divergence of both theories, we identify generic conditions for their renormalizability at all orders of perturbation at high momenta, i.e., the ultraviolet regime. For the first type of theory, we identify a 2-parameter space of just-renormalizable models for generic $(d, D)$. These models have dominant non-melonic four-point functions. Finally, by specifying the parameters, we detail the renormalization analysis of the second type of model which is more exotic: it exhibits an infinite family of logarithmically divergent two-point amplitudes and all four-point amplitudes are convergent. Published by AIP Publishing. https://doi.org/10.1063/1.5022438
\end{abstract}

\section{INTRODUCTION}

Tensor models, ${ }^{1-4}$ and their field theory version, tensor field theories are approaches to Quantum Gravity (QG) which propose a background-independent quantization and, in the field theory case, an ultraviolet-consistent completion of general relativity. They study a discrete-to-continuum transition for discretized path integrals summing over not only metrics of a discretized EinsteinHilbert action but also over topologies. The partition function of tensor models spans weighted triangulations for every piecewise-linear manifold in any dimensions; hence, they are naturally a random-geometric approach to QG. In this regard, they can be considered to fall under the umbrella of discretization approaches to QG, such as quantum Regge calculus ${ }^{5,6}$ and (causal) dynamical triangulations. ${ }^{7,8}$

Historically, tensor models were introduced as higher dimensional generalizations of matrix models which saw their celebrated success in describing 2-dimensional QG. ${ }^{9}$ It was, however, not so straightforward to generalize matrix models' achievements to higher dimensions. In particular, matrix models generate maps sorted by genus and their partition function then admits a genus expansion at a large size $N$ of the matrix. ${ }^{10}$ The large $N$ limit is crucial to achieve the continuum limit of matrix models, as a 2D theory of gravity coupled with a Liouville conformal field. ${ }^{11}$ This is one of the most acclaimed results pertaining to $2 \mathrm{D} \mathrm{QG}$.

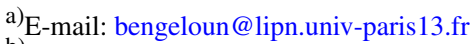

b) E-mail: reiko.toriumi@science.ru.nl
} 
The large $N$ limit for tensor models ${ }^{12,13}$ was finally unveiled after the advent of colored tensor models generating triangulations shown to be pseudo-manifolds. ${ }^{14-16}$ The partition function of colored tensor models can be catalogued in terms of a new quantity called the degree of the tensor graph which plays the role of the genus in higher dimensions. Such a discovery, as anticipated, led to a wealth of developments in random tensors in areas as diverse as statistical mechanics, quantum field theory (QFT), constructive field theory, combinatorics, probability theory, geometry, and topology ${ }^{17-54}$ (see also the following references which provide comprehensive reviews Refs. 22, 27, and 39). Furthermore, more recently, tensor models gathered attention in a new direction: they turn out to be desirable toy models for holographic duality ${ }^{55-59}$ The large $N$ limit in the range of the disorder of the famous Sachdev-Ye-Kitaev (SYK) model ${ }^{60-63}$ corresponds to the large $N$ limit of colored tensor models thought of as quantum mechanical models without disorder. ${ }^{55}$

Despite all its remarkable achievements, colored tensor models have not yet succeeded to define a "nice" continuum limit in which an emergent 3D or 4D space could be identified. In colored tensor models, some graphs which are particular triangulations of a sphere, called melons, are found to be dominant at large $N .{ }^{17}$ In the world of melons, colored tensor models undergo a phase transition towards the so-called branched polymer phase which is not of the characteristics (e.g., Hausdorff and spectral dimensions) that our large and smooth space-time manifold holds. ${ }^{35}$ In order to improve the critical behavior of tensor models, it was then put forward to go beyond the melonic sector, by modifying the weights of interactions in order to include a wider class of graphs that could be resummed at large $N$. Such a proposal has been called "enhancing" tensor models and has first been investigated in the work by Bonzom et al. ${ }^{36,37}$ The upshot of this analysis is encouraging: some enhanced tensor models undergo a phase transition from branched polymers to a 2D QG phase (with positive entropy exponents). Let us be more specific at this point: the previous studies on enhanced tensor models focused on increasing the statistical weights of non-melonic tensor interactions called necklaces (which are only present in the tensor rank $d \geq 4$ ). In a different perspective along with its very own set of questions, our proposal is to use the framework of field theories, therefore working in tensor field theories rather than in tensor models, and to explore new ways of building enhanced models in which non-melonic graphs could contribute to the analysis at large $N$.

Once one promotes tensor models to field theories, which possess now infinite degrees of freedom, we call them tensor field theories. Note that from the 90's, Boulatov introduced a gauge invariant version of tensor models by embedding them in lattice gauge field theory over $S U(2) .{ }^{4}$ This approach was considerably appealing to make contact with other QG approaches and was at the inception of Group Field Theory (GFT). ${ }^{64-66}$

There are other motivations for introducing fields in the search of an emergent spacetime. For instance, one makes another progress by regarding the simplicial complex associated with the tensor (in a tensor model) as a true (combinatorial) quantum of space. These fields live in an abstract internal space and are endowed with a given dynamics and consequently a flow. In this flow, fixed points would be associated with interesting physics. Thus, rather than tuning a given tensor model at criticality and seeing a new phase for geometry emerging, we might give initial conditions of a model in a field theory space and let it flow towards the corresponding fixed point. To define a flow, a parameter or a scale is needed. This makes the presence of propagators or regulators of paramount importance in the usual field theory. Hence embedding tensor models into a field theoretic context, that is, also giving them a propagator, provides them with a flow. This naturally steers us towards other interesting questions.

The Renormalization Group (RG) program has been successfully applied to tensor field theory and also GFT leading to the discovery of entirely new families of renormalizable non-local quantum field theories. ${ }^{40,43-47}$ These models can be regarded as a rightful extension of matrix field theories like the Grosse and Wulkenhaar model. ${ }^{67}$ The parametric representation and the ensuing dimensional regularization have been extended to tensor field theory with the emergence of new Symanzik polynomial invariants for tensor graphs. ${ }^{28}$ Moreover, the computations of the perturbative $\beta$-functions for $\phi^{4}$ - and $\phi^{6}$-like models were achieved in the ultraviolet (UV). ${ }^{42,43,48-50}$ The upshot of the perturbative analysis is that generically in tensor field theory, renormalizable $\phi^{4}$ models are UV asymptotically 
free, and renormalizable $\phi^{6}$ models are UV asymptotically safe. At the nonperturbative level, the exact renormalization group equations via Polchinski ${ }^{29,30}$ and via Wetterich [Functional Renormalization Group (FRG)] equations were fruitfully applied in all rank $d \geq 2$ matrix and tensor models with compelling corroborations on the existence of Gaussian and non-Gaussian UV and infrared (IR) points. ${ }^{25,31-33}$

In this paper, we undertake the study of the theory space of enhanced tensor field theories by addressing the perturbative renormalization of classes of enhanced models. We study tensor field models with quartic melonic interactions with a momentum weight mimicking derivative couplings. The effect of the new couplings is to make the non-melonic graph amplitudes larger than or as large as the melonic amplitudes. Note that derivative couplings are well established in ordinary renormalizable quantum field theory, e.g., appearing as in non-Abelian Yang-Mills theories. The issue addressed in this work is to set up tools which will allow one to find a class of renormalizable theories endowed with non-local and weighted interactions. As one can expect, the presence of these interactions bears additional subtleties as it naturally tends to increase the divergence degree of a graph.

The enhanced models that we study radically differ from that of Ref. 37 as we do not enhance non-melonic interactions of the necklace type but melonic interactions. Specifically, we focus on $\phi^{4}$-melonic couplings which are endowed with extra powers of momenta $|p|^{2 a}$; we call the resulting models $p^{2 a} \phi^{4}$-models, where $a \geq 0$ is a parameter. The study is put on a very general ground, at any rank $d$ of the tensor field defined on an Abelian group of dimension $d \times D$. The propagator of the model is of the form $\left(\sum|p|^{2 b}+\mu\right)^{-1}$, where $b>0$. Hence our model is parametrized by $(d, D$, $a, b)$. The case $a=0$ stands for the standard tensor field theory. Initially proposed by Ref. 38, these models were found tractable at fixed ranks $d=3,4, D=1$, and $b=1$, and there were indications of their super-renormalizability without a full-fledge proof of this statement. We carry on detailed analyses for these models, extending them at any rank and any dimension, and now prove rigorous theorems. The method that we use is the so-called multi-scale renormalization. ${ }^{68}$ Using this scheme, we then find conditions on the tuple $(d, D, a, b)$ for potentially renormalizable enhanced models of two different types.

- For the first type of theory, quite remarkably, we show that for generic $(d, D)$ parameters, there exists a just-renormalizable model at all orders. Theorem 1 summarizes this result.

- For the second type of theory, we prove the renormalizability at all orders of a specific model for a choice of parameters. Theorem 2 is another main result of our analysis.

We ought to specify that the entire analysis is performed in the UV, that is, at high momenta corresponding to short distances on the abstract Abelian group. The group being compact, we do not expect large domain divergences and hence no IR issues. However, if one uses a different renormalization scheme such that non-perturbative effects are being taken into account via the FRG equations, the study of the present models may bear interpretations which may be radically different from what we present here. In any case, restricting the upshot of these studies at small couplings should match with the present results.

The plan of the paper is as follows: In Sec. II, we introduce two models: the model + and the model $\times$ with different enhancements in the $\phi^{4}$-tensor interactions. In Sec. III, preparing for the power counting analyses, we give an explicit expression for the amplitude of a given Feynman graph. Section IV addresses the multi-scale analysis: we optimally bound a generic graph amplitude in terms of combinatorial quantities of the graph. In Sec. V, we determine the parameter spaces of $(d, D, a, b)$ which could potentially give rise to renormalizable models + and $\times$. Concretely, we investigate further instances of renormalizable models: (1) Section VI presents a generic model + with arbitrary $D, d, a=D(d-2) / 2$, and $b=D(2 d-3) / 4$; (2) Section VII addresses a model $\times$ with $D=1, d=3, a=1 / 2$, and $b=1$. We prove that these models determined by such parameters are indeed renormalizable at all orders of perturbation theory. We give a summary of our results and future prospectives in Sec. VIII. Closing the manuscript, in Appendix A, the reader will find the details of the spectral sums to be used for bounding the amplitudes, and Appendixes B and C, respectively, illustrate some representative and divergent graphs appearing in specific models + and $\times$. 


\section{ENHANCED $p^{2 a} \phi^{4}$ TENSOR FIELD THEORIES}

We consider a field theory defined by a rank $d$ complex tensor $\phi \mathbf{P}$, with $\mathbf{P}=\left(p_{1}, p_{2}, \ldots, p_{d}\right)$ a multi-index, and $\bar{\phi}_{\mathbf{P}}$ denotes its complex conjugate. From a field theory standpoint, introducing a complex function $\phi:\left(U(1)^{D}\right)^{\times d} \rightarrow \mathbb{C}$, where $D$ will be called the dimension of the group $U(1)^{D}, \phi_{\mathbf{P}}$ is the Fourier component of the field, and the indices $p_{s}$ are by themselves multi-indices

$$
p_{s}=\left(p_{s, 1}, p_{s, 2}, \ldots, p_{s, D}\right), p_{s, i} \in \mathbb{Z} .
$$

Let us make a few remarks. First, considering $\phi_{\mathbf{P}}$ as a rank $d$ tensor is a slight abuse because the modes $p_{k, s}$ range up to infinity. Cutting sharply off all modes to $N$, then the resulting multi-index object $\phi_{\mathbf{P} ; N}$ transforms under the fundamental representation of $U(N)^{D \times d}$ and hence is a tensor. For convenience, we keep the name of tensor for the field $\phi_{\mathbf{P}}$. Second, $\phi_{\mathbf{P}}$ could be considered as a $d \times D$ multi-index tensor; we shall call it a rank $d$ tensor because $d$ and $D$ will play different roles in the following. Third, several of the results derived hereafter could be extended to any compact Lie group $G_{D}$ of dimension $D$ admitting a Peter-Weyl decomposition (see, for instance, how a treatment for $S U(2)^{D^{\prime}}, D=3 D^{\prime}$, can be achieved using tools in Ref. 47). The treatment of the corresponding models could be achieved with some extra work. Finally, a last remark is that the dimension $D$ has nothing to do with the space dimension associated with the discrete geometry encoded by the tensor contractions as we will discuss soon. Thus referring in the following to UV and IR should be related with small and large distances on the group $U(1)^{D}$.

A general action $S$ built by a sum of convolutions of the tensors $\phi_{\mathbf{P}}$ and $\bar{\phi}_{\mathbf{P}}$ can be written as

$$
\begin{aligned}
& S[\bar{\phi}, \phi]=\operatorname{Tr}_{2}(\bar{\phi} \cdot \mathbf{K} \cdot \phi)+\mu \operatorname{Tr}_{2}\left(\phi^{2}\right)+S^{\mathrm{int}}[\bar{\phi}, \phi], \\
& \operatorname{Tr}_{2}(\bar{\phi} \cdot \mathbf{K} \cdot \phi)=\sum_{\mathbf{P}, \mathbf{P}^{\prime}} \bar{\phi}_{\mathbf{P}} \mathbf{K}\left(\mathbf{P} ; \mathbf{P}^{\prime}\right) \phi_{\mathbf{P}^{\prime}}, \quad \operatorname{Tr}_{2}\left(\phi^{2}\right)=\sum_{\mathbf{P}} \bar{\phi}_{\mathbf{P}} \phi \mathbf{P}, \\
& S^{\text {int }}[\bar{\phi}, \phi]=\sum_{\mathcal{B}} \lambda_{\mathcal{B}} \operatorname{Tr}_{\mathcal{B}}\left(\bar{\phi}_{\mathcal{B}}^{n} \cdot \mathbf{V}_{\mathcal{B}} \cdot \phi^{n_{\mathcal{B}}}\right),
\end{aligned}
$$

where $\mathcal{B}$ denotes for tensor invariant interactions, called "bubbles." $\operatorname{Tr}_{\mathcal{B}}$ are sums over all indices $p_{k, s}$ of $\mathbf{P}$ of $n_{\mathcal{B}}$ tensors $\phi$ and $\bar{\phi}$. Then $\operatorname{Tr}_{\mathcal{B}}$ are considered as traces over indices of the tensors. In (2), the kernels $\mathbf{K}$ and $\mathbf{V}_{\mathcal{B}}$ are to be specified, $\mu$ is a mass coupling, and $\lambda_{\mathcal{B}}$ is a coupling constant. If $\mathbf{V}_{\mathcal{B}}$ corresponds to a simple pairing between tensor indices (by delta functions identifying indices), then $\operatorname{Tr}_{\mathcal{B}}\left(\bar{\phi}^{n_{\mathcal{B}}} \cdot \mathbf{V}_{\mathcal{B}} \cdot \phi^{n_{\mathcal{B}}}\right)$ spans the space of unitary invariants. ${ }^{18,20,21}$ Note that for the moment, (2) is just notations and we will be precise on their notations shortly.

There is a geometrical interpretation of the interaction $\operatorname{Tr}_{\mathcal{B}}\left(\bar{\phi}^{n_{\mathcal{B}}} \cdot \mathbf{V}_{\mathcal{B}} \cdot \phi^{n_{\mathcal{B}}}\right)$. If each tensor field is regarded as a $d$-simplex, the generalized trace $\operatorname{Tr}_{n_{\mathcal{B}}}$ corresponds to a pairing or an identification of the $(d-1)$-simplices on the boundary of the $d$-simplexes to form a $d+1$ dimensional discrete geometry. If the kernel $\mathbf{V}_{\mathcal{B}}$ is not a simple pairing, it then assigns a weight to each of those discrete geometries.

A model is specified after giving the data of the kernels $\mathbf{K}$ and $\mathbf{V}_{\mathcal{B}}$. Let us introduce some convenient notations

$$
\begin{aligned}
& \boldsymbol{\delta}_{\mathbf{P} ; \mathbf{P}^{\prime}}=\prod_{s=1}^{d} \prod_{i=1}^{D} \delta_{p_{s, i}, p_{s, i}^{\prime}}, \quad \mathbf{P}^{2 b}=\sum_{s=1}^{d}\left|p_{s}\right|^{2 b}, \quad\left|p_{s}\right|^{2 b}=\sum_{i=1}^{D}\left|p_{s, i}\right|^{2 b}, \\
& \phi_{12 \ldots d}=\phi_{p_{1}, p_{2}, \ldots, p_{d}}=\phi_{\mathbf{P}}
\end{aligned}
$$

for a real parameter $b \geq 0$, and where $\delta_{p, q}$ is the usual Kronecker symbol on $\mathbb{Z}$.

We introduce the following class of kernels for the kinetic term

$$
\mathbf{K}_{b}\left(\mathbf{P} ; \mathbf{P}^{\prime}\right)=\delta_{\mathbf{P} ; \mathbf{P}^{\prime}} \mathbf{P}^{2 b} .
$$

$\mathbf{K}_{b}$ therefore represents a sum of the power of eigenvalues of $d$ Laplacian operators over the $d$ copies of $U(1)^{D}$. The case $b=1$ corresponds precisely to Laplacian eigenvalues on the torus. Seeking renormalizable theories, from the fact that we are dealing with a nonlocal model, we might be led to choose values of $b$ different from integers. In usual quantum field theory (QFT), $b$ should 
have an upper bound $b \leq 1$ to ensure the Osterwalder-Schrader (OS) positivity axiom. ${ }^{68}$ Whether or not such a condition (or any OS axioms) might be kept for tensor field theories is still in debate. ${ }^{27}$ Thus, for the moment, to avoid putting strong constraints on the models, we let $b$ as a free strictly positive real parameter.

We will be interested in 2 models distinguished by their interactions. Introduce a parameter $a \in$ $(0, \infty)$ and write

$$
\begin{aligned}
& \operatorname{Tr}_{4 ; 1}\left(\phi^{4}\right)=\sum_{p_{s}, p_{s}^{\prime} \in \mathbb{Z}^{D}} \phi_{12 \ldots d} \bar{\phi}_{1^{\prime} 23 \ldots d} \phi_{1^{\prime} 2^{\prime} 3^{\prime} \ldots d^{\prime}} \bar{\phi}_{12^{\prime} 3^{\prime} \ldots d^{\prime}}, \\
& \operatorname{Tr}_{4 ; 1}\left(\left[p^{2 a}+p^{\prime 2 a}\right] \phi^{4}\right)=\sum_{p_{s}, p_{s}^{\prime} \in \mathbb{Z}^{D}}\left(\left|p_{1}\right|^{2 a}+\left|p^{\prime}{ }_{1}\right|^{2 a}\right) \phi_{12 \ldots d} \bar{\phi}_{1^{\prime} 23 \ldots d} \phi_{1^{\prime} 2^{\prime} 3^{\prime} \ldots d^{\prime}} \bar{\phi}_{12^{\prime} 3^{\prime} \ldots d^{\prime}} \\
& =2 \sum_{p_{s}, p_{s}^{\prime} \in \mathbb{Z}^{D}}\left|p_{1}\right|^{2 a} \phi_{12 \ldots d} \bar{\phi}_{11^{\prime} 23 \ldots d} \phi_{1^{\prime} 2^{\prime} 3^{\prime} \ldots d^{\prime}} \bar{\phi}_{12^{\prime} 3^{\prime} \ldots d^{\prime}}=2 \operatorname{Tr}_{4 ; 1}\left(p^{2 a} \phi^{4}\right), \\
& \operatorname{Tr}_{4 ; 1}\left(\left[p^{2 a} p^{\prime 2 a}\right] \phi^{4}\right)=\sum_{p_{s}, p_{s}^{\prime} \in \mathbb{Z}^{D}}\left(\left|p_{1}\right|^{2 a}\left|p^{\prime}{ }_{1}\right|^{2 a}\right) \phi_{12 \ldots d} \bar{\phi}_{1^{\prime} 23 \ldots d} \phi_{1^{\prime} 2^{\prime} 3^{\prime} \ldots d^{\prime}} \bar{\phi}_{12^{\prime} 3^{\prime} \ldots d^{\prime}} .
\end{aligned}
$$

Note that in (5)-(7), the color index 1 plays a special role. We stress that the left-hand side of these equations presents in a very schematic notation complicated patterns of weighted convolutions of tensors. We however keep these shorthand notations for convenience. Then, summing over all possible color indices, one obtains colored symmetric interactions

$$
\begin{aligned}
& \operatorname{Tr}_{4}\left(\phi^{4}\right):=\operatorname{Tr}_{4 ; 1}\left(\phi^{4}\right)+\operatorname{Sym}(1 \rightarrow 2 \rightarrow \cdots \rightarrow d), \\
& \operatorname{Tr}_{4}\left(p^{2 a} \phi^{4}\right):=\operatorname{Tr}_{4 ; 1}\left(p^{2 a} \phi^{4}\right)+\operatorname{Sym}(1 \rightarrow 2 \rightarrow \cdots \rightarrow d), \\
& \operatorname{Tr}_{4}\left(\left[p^{2 a} p^{\prime 2 a}\right] \phi^{4}\right):=\operatorname{Tr}_{4 ; 1}\left(\left[p^{2 a} p^{\prime 2 a}\right] \phi^{4}\right)+\operatorname{Sym}(1 \rightarrow 2 \rightarrow \cdots \rightarrow d) .
\end{aligned}
$$

The momentum weights in the interactions $\operatorname{Tr}_{4}\left(p^{2 a} \phi^{4}\right)$ and $\operatorname{Tr}_{4}\left(\left[p^{2 a} p^{2 a}\right] \phi^{4}\right)$ can be viewed as derivative couplings for particular choices of $a$. This is why, at times, we will call them derivative couplings. Written in the momentum space, the interactions are however put in a more general setting using $|p|^{2 a}$, for positive values of $a$. Once again, achieving renormalizability will be our sole constraint for fixing $a$. These interactions are called enhanced compared to $\operatorname{Tr}_{4}\left(\phi^{4}\right)$ (the usual quartic melonic graph studied for instance in Ref. 42) because they can generate amplitudes which are more divergent, and so enhanced, compared to those generated by $\operatorname{Tr}_{4}\left(\phi^{4}\right)$ alone.

The interactions that we consider in (8) are called melonic bubbles. They are represented by bi-partite colored graphs, see Fig. 2, however, with momentum enhancement on the "weak" edges of melonic bubbles that we now define. Having a look on the graphs representing melonic bubbles, Fig. 2, one singles out two particular edges which separate the graph in two sectors made of two pairs of vertices having multiple edges (also called dipoles). We call the separating edges, "weak" links or edges of the melonic bubble interaction. In Fig. 2, the weak links appear as vertical lines with color 1. Anticipating a bit on the quantum perturbative analysis of Feynman graphs and their amplitudes, we justify the rationale behind putting a weight on weak links. The most divergent graphs in standard tensor field theory are melonic graphs which maximize the number of cycles in these graphs. In a tensor field theory built with $\phi^{4}$ melonic interactions, melonic graphs maximize the number of cycles made with the multiple edges and minimize cycles involving the weak links. In opposition, to generate non-melonic graphs in such theories, one necessarily needs to close a maximum of weak sectors. In an ordinary non-enhanced model, the amplitudes of non-melonic graphs are smaller than those of melonic graphs. In order to go beyond a regime where only melons dominate, as motivated in the Introduction, we therefore need to increase the contribution of the cycles going through weak links in the melonic interactions. We achieve this by putting more momentum weights on the weak sectors in these melonic bubbles in such a way that non-melonic graphs which are made of these enhanced melonic bubbles come with a divergence degree larger than or at least equal to that of melonic graphs (built with identical interactions). As a second property, we discussed that enhanced interactions represent weighted discrete geometries. The contraction pattern of the four tensors shows us that 
the weight here has a subtle sense: we are weighting a particular $(d-1)$-simplex in the $(d+1)$-simplex representing the interaction.

It turns out that the renormalization analysis performed in Secs. VI and VII leads us to new 2-point diverging graphs. Then we must add to the kinetic term the new terms

$$
\operatorname{Tr}_{2}\left(p^{2 \xi} \phi^{2}\right)=\operatorname{Tr}_{2}\left(\bar{\phi} \cdot \mathbf{K}_{\xi} \cdot \phi\right), \quad \xi=a, 2 a,
$$

in addition to the kinetic term $\operatorname{Tr}_{2}\left(p^{2 \xi} \phi^{2}\right)$, where $\xi=b$.

We will need counter-terms for each term in the action. In particular, the counter-term $C T_{2}$ of the form of the mass, $C T_{2 ; b}$ for the wave function, and new 2-point interactions $C T_{2 ; a}$ and $C T_{2 ; 2 a}$, will be important for renormalizing two-point functions. We define

$$
C T_{2}[\bar{\phi}, \phi]=\delta_{\mu} \operatorname{Tr}_{2}\left(\phi^{2}\right), C T_{2 ; \xi}[\bar{\phi}, \phi]=Z_{\xi} \operatorname{Tr}_{2}\left(p^{2 \xi} \phi^{2}\right), \xi=a, 2 a, b,
$$

where $\delta_{\mu}$ and $Z_{\xi}$ are counter-term couplings. Note that in the following, $Z_{b}$ is called wave function renormalization.

The models that we will study have the following kinetic terms and interactions:

$$
\begin{aligned}
\text { model }+: \quad S_{+}^{\text {int }}[\bar{\phi}, \phi] & =\frac{\lambda}{2} \operatorname{Tr}_{4}\left(\phi^{4}\right)+\frac{\eta_{+}}{2} \operatorname{Tr}_{4}\left(p^{2 a} \phi^{4}\right)+C T_{2}[\bar{\phi}, \phi]+\sum_{\xi=a, b} C T_{2 ; \xi}[\bar{\phi}, \phi] \\
S_{+}^{\mathrm{kin}}[\bar{\phi}, \phi] & =\sum_{\xi=a, b} \operatorname{Tr}_{2}\left(p^{2 \xi} \phi^{2}\right)+\mu \operatorname{Tr}_{2}\left(\phi^{2}\right), \\
\text { model } \times: \quad S_{\times}^{\text {int }}[\bar{\phi}, \phi] & =\frac{\lambda}{2} \operatorname{Tr}_{4}\left(\phi^{4}\right)+\frac{\eta_{\times}}{2} \operatorname{Tr}_{4}\left(\left[p^{2 a} p^{\prime 2 a}\right] \phi^{4}\right)+C T_{2}[\bar{\phi}, \phi]+\sum_{\xi=a, 2 a, b} C T_{2 ; \xi}[\bar{\phi}, \phi], \\
S_{\times}^{\mathrm{kin}}[\bar{\phi}, \phi] & =\sum_{\xi=a, 2 a, b} \operatorname{Tr}_{2}\left(p^{2 \xi} \phi^{2}\right)+\mu \operatorname{Tr}_{2}\left(\phi^{2}\right),
\end{aligned}
$$

where $\lambda, \eta_{+}$, and $\eta_{\times}$are coupling constants.

It is an interesting question to list the classical symmetries of the models + and $\times$ given by the generalized Noether theorem for such non-local theories. ${ }^{51,52}$ To apply the Lie symmetry algorithm as worked out in these references could be an interesting exercise for derivative coupling theories and could bear important consequences for the Ward identities.

The present theory space is clearly much more involved than the usual unitary invariant theory space where the vertices of the model do not have any momentum weight. It will result from our analysis that a new combinatorics provides our models with a genuinely different renormalization procedure. Then, the comparison could be made with the models in Table 8 in Ref. 47 which are unitary invariant models. We seize this opportunity to correct that table: the justrenormalizable $\phi^{6}$-models should be UV asymptotically safe (rather than free) under the light of recent results. $^{24,26,34,48}$

\section{AMPLITUDES}

Models + and $\times$ associated with actions given by (11) and (12), respectively, give the quantum models determined by the partition function

$$
Z_{\bullet}=\int d v_{C_{\bullet}}(\bar{\phi}, \phi) e^{-S_{\bullet}^{\text {int }}[\bar{\phi}, \phi]},
$$

where $\bullet=+, \times$, and $d v_{C}(\bar{\phi}, \phi)$ is a field Gaussian measure with covariance $C \bullet$ given by the inverse of the kinetic term

$$
C_{\bullet}\left(\mathbf{P} ; \mathbf{P}^{\prime}\right)=\tilde{C}_{\bullet}(\mathbf{P}) \delta_{\mathbf{P}, \mathbf{P}^{\prime}}, \quad \tilde{C}_{\bullet}(\mathbf{P})=\frac{1}{\sum_{\xi} \mathbf{P}^{2 \xi}+\mu},
$$


where if $\bullet=+, \xi=a, b$ and if $\bullet=\times, \xi=a, 2 a, b$. Dealing with the interactions, we have the vertex kernels $\mathbf{V}_{4 ; s}$ and $\mathbf{V}_{+; 4 ; s}$ associated with (11) and $\mathbf{V}_{4 ; s}$ and $\mathbf{V}_{\times ; 4 ; s}$ associated with (12). These kernels are given by

$$
\begin{aligned}
& \mathbf{V}_{4 ; s}\left(\mathbf{P} ; \mathbf{P}^{\prime} ; \mathbf{P}^{\prime \prime} ; \mathbf{P}^{\prime \prime \prime}\right)=\frac{\lambda}{2} \delta_{4 ; s}\left(\mathbf{P} ; \mathbf{P}^{\prime} ; \mathbf{P}^{\prime \prime} ; \mathbf{P}^{\prime \prime \prime}\right), \\
& \mathbf{V}_{+; 4 ; s}\left(\mathbf{P} ; \mathbf{P}^{\prime} ; \mathbf{P}^{\prime \prime} ; \mathbf{P}^{\prime \prime \prime}\right)=\frac{\eta_{+}}{2}\left|p_{s}\right|^{2 a} \delta_{4 ; s}\left(\mathbf{P} ; \mathbf{P}^{\prime} ; \mathbf{P}^{\prime \prime} ; \mathbf{P}^{\prime \prime \prime}\right), \\
& \mathbf{V}_{\times ; 4 ; s}\left(\mathbf{P} ; \mathbf{P}^{\prime} ; \mathbf{P}^{\prime \prime} ; \mathbf{P}^{\prime \prime \prime}\right)=\frac{\eta_{\times}}{2}\left|p_{s}\right|^{2 a}\left|p_{s}^{\prime}\right|^{2 a} \delta_{4 ; s}\left(\mathbf{P} ; \mathbf{P}^{\prime} ; \mathbf{P}^{\prime \prime} ; \mathbf{P}^{\prime \prime \prime}\right),
\end{aligned}
$$

$s=1,2, \ldots, d$, where the operator $\delta_{4 ; s}(-)$ is a product of Kronecker deltas identifying the different momenta according to the pattern dictated by the interaction $\operatorname{Tr}_{4 ; s}\left(\phi^{4}\right)$. Note that $\mathbf{V}_{\bullet ; 4 ; s}$ has a color index. The vertex operator $\mathbf{V}_{2}$ associated with the mass counter-term is a delta function $\boldsymbol{\delta}_{\mathbf{P} ; \mathbf{P}^{\prime}} ;$ the vertex operators $\mathbf{V}_{2 ; \xi ; s} \xi=a, 2 a, b$, associated with the counter-terms $C T_{2 ; \xi}[\bar{\phi}, \phi]$, are delta functions weighted by momenta $\left|p_{s}\right|^{2 \xi}$.

\section{A. Feynman tensor graphs}

There are two equivalent graphical representations of Feynman graphs in tensor models. The first one is called "stranded graph" and it incorporates more details of the structure of the Feynman graph (used and explained in Refs. 14 and 47). The other representation of a Feynman graph in this theory is a bipartite colored graph. ${ }^{14,16,20,35}$ We mostly use the latter because it is convenient and economic. The first representation will be used in this section to make explicit the notion of faces associated with momentum loops.

At the graphical level, the propagator is drawn as a collection of $d$ segments called strands (see Fig. 1).

Each interaction is sketched as a stranded vertex or by a $d$-regular colored bipartite graph called a "bubble." The bipartiteness of the graph comes from the representation of each field $\phi$ as a white vertex and each field $\bar{\phi}$ by a black one. For instance, see the bubbles corresponding to $\phi^{2}$ vertices (mass and wave functions vertices) and $\phi^{4}$-interactions in Fig. 2 . Note that, the bubbles representing the vertex kernel $\mathbf{V}_{\bullet ; 4 ; s}, \bullet=+, \times$, appear with one or two bold edges, respectively. The color of a bold edge corresponds to the color index of the enhanced momentum. The bubbles that describe the vertices are particular contractions of tensors and are called melons.

Perturbation theory tells us that, via the Wick theorem, we should glue vertices by propagator lines to produce a Feynman graph. Some examples of Feynman tensor graphs by the above rule are depicted in Fig. 3. We put half-lines or external legs on vertices to reflect the presence of external fields. In the following, a Feynman tensor graph is simply called a graph and is denoted by $\mathcal{G}$.

In the stranded picture, closed cycles (homeomorphic to circles) in the graphs are called closed or internal faces and strands homeomorphic to segments are called open or external faces. The set of closed faces is denoted by $\mathcal{F}_{\text {int }}$ and the set of open faces $\mathcal{F}_{\text {ext }}$. As expected, the presence of an internal face is associated with a sum over infinite values of momenta which can make the amplitude divergent, hence the need of regularization and renormalization for the model. In the colored graph representation, note that an extra color 0 could be attributed to all dotted propagator lines. The cycles in that $(d+1)$ colored graph have two colors. The internal faces of $\mathcal{G}$, elements of $\mathcal{F}_{\text {int }}$, are associated with bicolored cycles of colors $0 s$, with $s=1,2, \ldots, d$. To obtain the subset of $\mathcal{F}_{\text {int }}$ (or of $\mathcal{F}_{\text {ext }}$ ) of faces of colors $0 s$ from the $d+1$ colored graph, we remove all edges except those of colors 0 and $s$ and observe the remaining cycles (or open strands, respectively). In the end, for

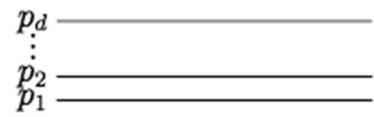

FIG. 1. The propagator of the theory: the stranded representation (left) made with $d$ segments representing $d$ momenta; the colored representation (right) denoted by a dotted line. 

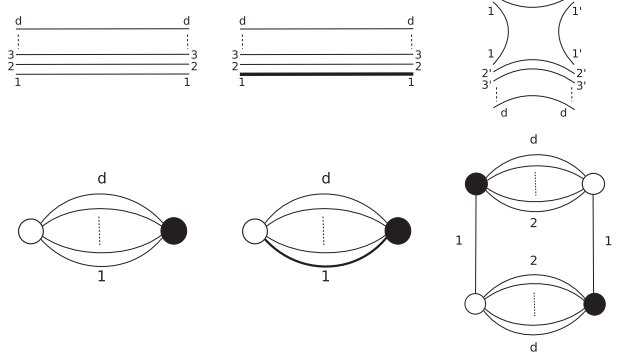

$\operatorname{Tr}_{2}\left(\Phi^{2}\right)$

$\operatorname{Tr}_{2}\left(p_{1}^{2 \xi} \Phi^{2}\right)$
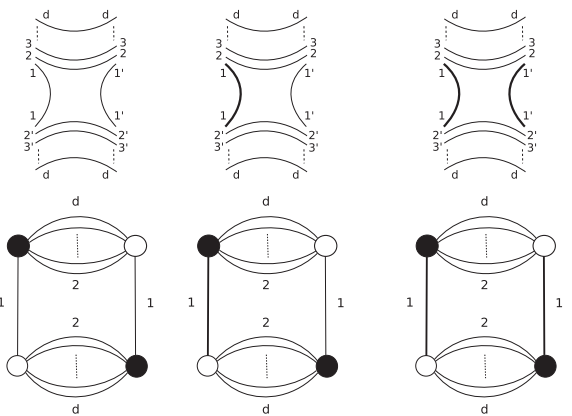

$\operatorname{Tr}_{4 ; 1}\left(p_{1}^{2 a} \Phi^{4}\right) \operatorname{Tr}_{4 ; 1}\left(p_{1}{ }^{2 a} p_{1}{ }^{2 a} \Phi^{4}\right)$

FIG. 2. Rank $d$ vertices of the mass, $\phi^{2}$-and $\phi^{4}$-terms.

simplicity, we omit the color 0 in the couple $0 s$ and claim that a (internal or external) face is of color $s$.

\section{B. Amplitudes}

Given a connected graph $\mathcal{G}$ with vertex set $\mathcal{V}$ (with $V=|\mathcal{V}|$ ) and line or propagator set $\mathcal{L}$ (with $L=|\mathcal{L}|)$, we formally write the amplitude of $\mathcal{G}$

$$
A_{\mathcal{G}}=\sum_{\mathbf{P}_{v}} \prod_{l \in \mathcal{L}} C_{\bullet ; l}\left(\mathbf{P}_{v(l)} ; \mathbf{P}_{v^{\prime}(l)}^{\prime}\right) \prod_{v \in \mathcal{V}}\left(-\mathbf{V}_{v}\left(\left\{\mathbf{P}_{v}\right\}\right)\right) .
$$

The above formula shows that propagators $C_{l}$ have a line index $l$ and momentum arguments $\mathbf{P}_{v(l)}$, with $v(l)$ the source or target of the line $l$. The vertex constraints $\mathbf{V}_{v}$ convolute the set of momenta and can be of the form $\mathbf{V}_{4 ; s}, \mathbf{V}_{\bullet ; 4 ; s}, \mathbf{V}_{2}, \mathbf{V}_{2 ; \xi ; s}, \xi=a, 2 a, b$. The presence of these weights makes the amplitude quite different from those of unitary invariant theories. For instance, as opposed to the ordinary situation, the amplitudes do not directly factorize in terms of internal faces.

To derive a power counting theorem, we need to study graph amplitudes $A_{\mathcal{G}}$ coming from the perturbative expansion of correlators of the form

$$
\begin{aligned}
& \left\langle\phi_{\mathbf{P}} \bar{\phi}_{\mathbf{P}^{\prime}} \phi_{\mathbf{P}^{\prime}} \bar{\phi}_{\mathbf{P}^{\prime \prime \prime}}\right\rangle, \\
& \left\langle\left|p_{1}\right|^{2 a} \phi_{\mathbf{P}} \bar{\phi}_{\mathbf{P}^{\prime}} \phi_{\mathbf{P}^{\prime}} \bar{\phi}_{\mathbf{P}^{\prime \prime \prime}} \delta_{4 ; s}\left(\mathbf{P} ; \mathbf{P}^{\prime} ; \mathbf{P}^{\prime \prime} ; \mathbf{P}^{\prime \prime \prime}\right)\right\rangle, \\
& \left\langle\left|p_{1}\right|^{2 a}\left|p_{1^{\prime}}\right|^{2 a} \phi_{\mathbf{P}} \bar{\phi}_{\mathbf{P}^{\prime}} \phi_{\mathbf{P}^{\prime}} \bar{\phi}_{\mathbf{P}^{\prime \prime \prime}} \delta_{4 ; s}\left(\mathbf{P} ; \mathbf{P}^{\prime} ; \mathbf{P}^{\prime \prime} ; \mathbf{P}^{\prime \prime \prime}\right)\right\rangle .
\end{aligned}
$$

In tensor graphs, consider the faces as previously introduced. A face $f_{s}$ with color $s$ has an $s$ colored $\mathbb{Z}^{D}$ conserved momentum $p_{f_{s}}$ and passes through some vertices $v_{s}$, with vertex kernel of the
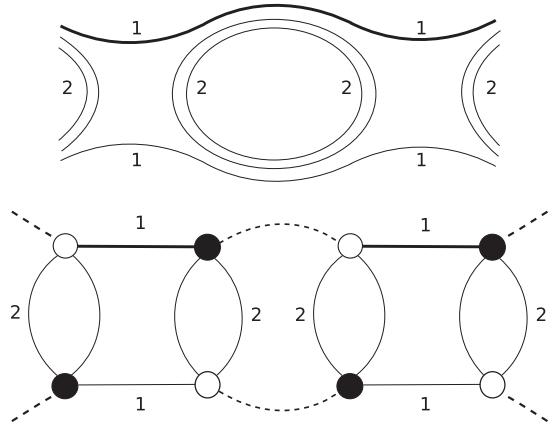
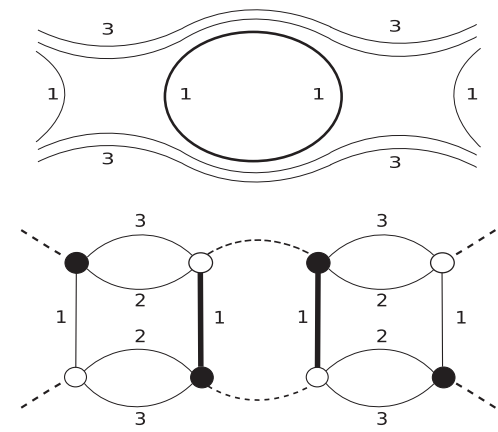

FIG. 3. Rank $d=3$ Feynman graphs. 
form $\mathbf{V}_{4 ; s}, \mathbf{V}_{\bullet ; 4 ; s}, \mathbf{V}_{2}$, or $\mathbf{V}_{2 ; \xi ; s}, \xi=a, 2 a, b$. This face may also pass through some other vertices with color $s^{\prime} \neq s$. More generally, a face $f$ can pass through a vertex $v$ a number of times, say, $\alpha$. Denote this statement by $v^{\alpha} \in f$. Because of the coloring, $\alpha$ can only be $0,1,2\left(v_{s} \in f_{s}\right.$ will mean $v_{s}^{1} \in f_{s}$ ). We therefore define the incidence matrix between faces and vertices by

$$
\epsilon_{v_{s} f_{s^{\prime}}}= \begin{cases}\alpha, & \left(s=s^{\prime}\right) \wedge\left(v_{s}^{\alpha} \in f_{s}\right), \\ 0, & \text { otherwise. }\end{cases}
$$

Given two faces $f_{1 ; s_{1}}$ and $f_{2 ; s_{2}}$ and a vertex $v_{s}$, we introduce another multi-index object that we denote by $\epsilon_{v_{\mathrm{s}} f_{1 ; s_{1}} f_{2 ; s_{2}}}$ defined as

$$
\epsilon_{v_{s_{1}} f_{1 ; s_{1}} f_{2 ; s_{2}}}= \begin{cases}1, & \left(s=s_{1}=s_{2}\right) \wedge\left(v_{s} \in f_{1 ; s_{1}}\right) \wedge\left(v_{s} \in f_{2 ; s_{2}}\right), \\ 0, & \text { otherwise. }\end{cases}
$$

The case $f_{1 ; s_{1}}=f_{2 ; s_{2}}$ could also occur. A first observation is that $\epsilon_{v_{s} f_{1 ; 1}} f_{2 ; s_{2}}=\epsilon_{v_{s} f_{s_{1}}} \epsilon_{v_{s} f_{s_{2}}}$ in the case when $v_{s} \in f_{1 ; s_{1}}$ and $v_{s} \in f_{2 ; s_{2}}$. Looking at the diagonal, i.e., $f_{1 ; s_{1}}=f_{2 ; s_{2}}, \epsilon_{v_{s}} f_{1 ; s} f_{1 ; s}=1$ if and only if $v_{s}^{2} \in f_{1 ; s}$.

We are in position to re-express the interaction weights $\mathbf{V}_{\bullet ; 4 ; s}$ in (15). Fix a color $s$; the weight of a vertex kernel of $v_{s}$ of the kind $\mathbf{V}_{\bullet ; 4 ; s}$ can be written as

$$
\begin{array}{ll}
\text { model }+: & \frac{\eta_{+}}{2} \sum_{f_{s^{\prime}}} \epsilon_{v_{s}, f_{s^{\prime}}}\left|p_{f_{s^{\prime}}}\right|^{2 a}, \\
\text { model } \times: & \frac{\eta_{\times}}{2} \sum_{f_{s^{\prime}}, f_{s^{\prime \prime}}} \epsilon_{v_{s}, f_{s^{\prime}}, f_{s^{\prime \prime}}}\left|p_{f_{s^{\prime}}}\right|^{2 a}\left|p_{f_{s^{\prime \prime}}}\right|^{2 a} .
\end{array}
$$

We stress, at this point, that the two models + and $\times$ will be studied separately; then, there is no confusion to adopt a single notation as

$$
\text { model } \bullet: \quad \frac{\eta}{2}(\epsilon p)_{v_{s}} \text {. }
$$

The weight of degree 2 vertices (in both models) which belong to $\mathcal{V}_{2 ; \xi ; s}$ is of the form $Z_{\xi} \sum_{f_{s}} \epsilon_{v_{s}}, f_{s^{\prime}}\left|p_{f_{s^{\prime}}}\right|^{2 \xi}=Z_{\xi}(\epsilon p)_{v_{s}}$, where $\xi=a, 2 a, b$.

Let us introduce

- the set $\mathcal{V}_{4 ; s}$ of vertices with kernel $\mathbf{V}_{4 ; s}, \mathcal{V}_{4}=\sqcup_{s=1}^{d} \mathcal{V}_{4 ; s}$ (disjoint union notation),

- the set $\mathcal{V}_{\bullet ; 4 ; s}$ of vertices with vertex kernel $\mathbf{V}_{\bullet ; 4 ; s}, \bullet=+, \times, \mathcal{V}_{\bullet ; 4}=\sqcup_{s=1}^{d} \mathcal{V}_{\bullet ; 4 ; s}$, and

- the set $\mathcal{V}_{2}$ of mass vertices with kernel $\mathbf{V}_{2}$, the set $\mathcal{V}_{2 ; \xi ; s}$ of vertices with kernels $\mathbf{V}_{2 ; \xi ; s}, \mathcal{V}_{2 ; s}$ $=\cup_{\xi} \mathcal{V}_{2 ; \xi ; s}$.

We denote the cardinalities $\left|\mathcal{V}_{4 ; s}\right|=V_{4 ; s},\left|\mathcal{V}_{4}\right|=V_{4},\left|\mathcal{V}_{\bullet ; 4 ; s}\right|=V_{\bullet ; 4 ; s},\left|\mathcal{V}_{\bullet ; 4}\right|=V_{\bullet ; 4}, \bullet=+, \times$ and $\left|\mathcal{V}_{2 ; \xi ; s}\right|=V_{2 ; \xi ; s}, V_{2 ; \xi}=\sum_{s} V_{2 ; \xi ; s}$. Then, $\mathcal{V}=\sqcup_{s=1}^{d}\left(\mathcal{V}_{4 ; s} \cup \mathcal{V}_{\bullet ; 4 ; s} \cup \mathcal{V}_{2 ; s}\right)$, and $|\mathcal{V}|=V$.

Using the Schwinger parametric form of the propagator kernel as

$$
\tilde{C}_{\bullet}(\mathbf{P})=\int_{0}^{\infty} d \alpha e^{-\alpha\left(\sum_{\xi} \mathbf{P}^{2 \xi}+\mu\right)},
$$

integrating all deltas from propagators and vertex operators, we put the amplitude (16) in the form

$$
\begin{aligned}
& A_{\mathcal{G}}=\kappa\left(\lambda, \eta_{\bullet}, Z_{\xi}\right) \sum_{p_{f_{s}}} \int\left[\prod_{l \in \mathcal{L}} d \alpha_{l} e^{-\alpha_{l} \mu}\right]\left[\prod_{f_{s} \in \mathcal{F}_{\text {ext }}} e^{-\left(\sum_{l \in f_{s}} \alpha_{l}\right) \sum_{\xi}\left|p_{f_{s}}^{\mathrm{ext}}\right|^{2 \xi}}\right] \\
& \times\left[\prod_{f_{s} \in \mathcal{F}_{\text {int }}} e^{-\left(\sum_{l \in f_{s}} \boldsymbol{\alpha}_{l}\right) \sum_{\xi}\left|p_{f_{s}}\right|^{2 \xi}}\right]\left[\prod_{s=1}^{d} \prod_{v_{s} \in \mathcal{V}_{\bullet ; ; ; s} \cup \mathcal{V}_{2 ; s}}(\epsilon \tilde{p})_{v_{s}}\right] \text {, }
\end{aligned}
$$

where $\kappa\left(\lambda, \eta_{\bullet}, Z_{\xi}\right)$ includes symmetry factors and coupling constants and $p_{f_{s}}^{\text {ext }}$ are external momenta which are not summed, whereas $p_{f_{s}}$ are internal momenta and are summed. In the last line, $\tilde{p}_{f_{s}}$ refers to an internal or an external momentum. The sum over infinite values of momenta produces divergent 
amplitudes (25). In Sec. IV, we will address the nature of these divergences through a power counting theorem.

\section{POWER COUNTING THEOREMS FOR $p^{2 a} \phi^{4}$-MODELS}

For simplicity, we will study the amplitude of a connected graph without $\mathcal{V}_{2 ; \xi ; s}$ vertices. Adding these vertices towards the end can be easily done.

\section{A. Multiscale analysis}

We slice the propagator in a geometric progression with the parameter $M>1$ and then bound each slice of the propagator

$$
\begin{aligned}
& \tilde{C}_{\bullet}(\mathbf{P})=\int_{0}^{\infty} d \alpha e^{-\alpha\left(\sum_{\xi} \mathbf{P}^{2 \xi}+\mu\right)}=\sum_{i=0}^{\infty} C_{\bullet ; i}(\mathbf{P}), \\
& C_{\bullet ; 0}(\mathbf{P})=\int_{1}^{\infty} d \alpha e^{-\alpha\left(\sum_{\xi} \mathbf{P}^{2 \xi}+\mu\right)} \leq K, \\
& C_{\bullet ; i}(\mathbf{P})=\int_{M^{-2(i+1)}}^{M^{-2 i}} d \alpha e^{-\alpha\left(\sum_{\xi} \mathbf{P}^{2 \xi}+\mu\right)} \leq K^{\prime} M^{-2 i} e^{-M^{-2 i}\left(\sum_{\xi} \mathbf{P}^{2 \xi}+\mu\right)} \\
& \leq K M^{-2 i} e^{-\delta M^{-i}\left(\sum_{\xi} \sum_{s=1}^{d} \sum_{l=1}^{D} \mid p_{s, l} l^{\xi}+\mu\right)} \leq K M^{-2 i} e^{-\delta M^{-i}\left(\sum_{\xi} \sum_{s}\left|p_{s}\right|^{\xi}+\mu\right)},
\end{aligned}
$$

$\left|p_{s}\right|^{\xi}=\sum_{l=1}^{D}\left|p_{s ; l}\right|^{\xi}$, for some constants $K, K^{\prime}$, and $\delta$.

The slice decomposition yields the standard interpretation that high values of $i$ select high momenta of order $\sim M^{i}$ and this refers to the UV (this coincides with small distances on the group $\left.U(1)^{D}\right)$. Meanwhile, low momenta are picked around the slice $i=0$ and correspond to the IR. Note that since we are dealing with a compact group, the latter limit is harmless. Introduce a cutoff $\Lambda$ on the slices $i$ and then cutoff the propagators as $C_{\bullet}^{\Lambda}=\sum_{i=0}^{\Lambda} C_{\bullet ; i}$. We will not display $\Lambda$ in the following expression.

Cutting off all propagators in (16), the amplitude $A_{\mathcal{G}}$ becomes $\sum_{\boldsymbol{\mu}} A_{\mathcal{G} ; \boldsymbol{\mu}}$ where $\boldsymbol{\mu}=\left\{i_{l}\right\}_{l \in \mathcal{L}}$ is a multi-index called momentum assignment which collects the propagator indices $i_{l} \in[0, \Lambda]$ and

$$
A_{\mathcal{G} ; \boldsymbol{\mu}}=\kappa\left(\lambda, \eta_{\bullet}\right) \sum_{p_{v ; s}}\left[\prod_{l \in \mathcal{L}} C_{\bullet ; i_{l}}\left(\mathbf{P}_{v(l)} ; \mathbf{P}_{v^{\prime}(l)}^{\prime}\right)\right]\left[\prod_{s=1}^{d} \prod_{v_{s} \in \mathcal{V}_{\bullet ; ; ; s}}(\epsilon \tilde{p})_{v_{s}}\right]
$$

Using (26), the above expression finds the form

$$
\begin{aligned}
& \left|A_{\mathcal{G} ; \boldsymbol{\mu}}\right| \leq \kappa\left(\lambda, \eta_{\bullet}\right) K^{L} K_{1}^{V} K_{2}^{F_{\text {ext }}}\left[\prod_{l \in \mathcal{L}} M^{-2 i_{l}}\right] \\
& \times \sum_{p_{f_{s}}}\left[\prod_{f_{s} \in \mathcal{F}_{\text {int }}} e^{-\left.\delta\left(\sum_{l \in \epsilon_{s}} M^{-i_{l}}\right) \sum_{\xi}\left|p_{f_{s}}\right|\right|^{\xi}}\right]\left[\prod_{s=1}^{d} \prod_{v_{s} \in \mathcal{V}_{\bullet ; 4 ; s}}(\epsilon \tilde{p})_{v_{s}}\right],
\end{aligned}
$$

where $K_{1,2}$ are constants.

$A_{\mathcal{G} ; \boldsymbol{\mu}}$ is the focus of our attention and is the quantity that must be bounded by an optimization procedure. A standard procedure detailed in Ref. 68 will allow one to sum over the assignments $\mu$ after renormalization.

The next definition can be found in Ref. 68. It paves the way to the notion of the locality of the theory through the definition of quasi-local subgraphs. Let $\mathcal{G}$ be a graph, with the line set $\mathcal{L}$. Fix $i$ as a slice index and define $\mathcal{G}^{i}$ to be the subgraph of $\mathcal{G}$ built with propagator lines with indices obeying $\forall \ell \in \mathcal{L}\left(\mathcal{G}^{i}\right) \cap \mathcal{L}, i_{\ell} \geq i$. It might happen that $\mathcal{G}^{i}$ disconnects in several components; 
we denote these connected components $G_{k}^{i}$ and call them quasi-local subgraphs. It is important to give a characterization of the quasi-local subgraphs. Given $g$, a subgraph of $\mathcal{G}$ with a internal line set $\mathcal{L}(g)$ and a external line set $\mathcal{L}_{\text {ext }}(g)$. Consider a momentum assignment $\boldsymbol{\mu}$ of $\mathcal{G}$ and define $i_{g}(\boldsymbol{\mu})$ $=\inf _{\ell \in \mathcal{L}(g)} i_{\ell}$ and $e_{g}(\boldsymbol{\mu})=\sup _{\ell \in \mathcal{L}_{\text {ext }}(g)} i_{\ell}$. We can identify $g$ with a quasi-local subgraph of $\mathcal{G}$ if and only if $i_{g}(\boldsymbol{\mu})>e_{g}(\boldsymbol{\mu})$.

The set $\left\{G_{k}^{i}\right\}$ of quasi-local subgraphs of $\mathcal{G}$ is partially ordered under inclusion. The inclusion can be put in a form of an abstract tree (with the vertices $G_{k}^{i}$,s) called the Gallavotti-Nicolò (GN) tree. ${ }^{69}$ We perform the internal momenta sums in (28) in an optimal way and show that the result can be expressed in terms of the quasi-local subgraphs. This condition, called the compatibility condition with the GN tree, turns out to be crucial when performing the sum over the momentum attribution.

All external momenta must be at a lower scale than internal momenta, and thus for any external faces $f_{s}$ and internal face $f_{s^{\prime}}, p_{f_{s}}^{\text {ext }} \ll p_{f_{s^{\prime}}}$. We bound all factors or terms with $p_{f_{s}}^{\text {ext }}$ and obtain

$$
\left|A_{\mathcal{G} ; \boldsymbol{\mu}}\right| \leq K_{3}\left[\prod_{l \in \mathcal{L}} M^{-2 i_{l}}\right] \sum_{p_{f_{s}}}\left[\prod_{f_{s} \in \mathcal{F}_{\text {int }}} e^{-\delta\left(\sum_{l \in f_{s}} M^{-i}\right) \sum_{\xi}\left|p_{f_{s}}\right| \xi}\right]\left[\prod_{s=1}^{d} \prod_{v_{s} \in \mathcal{V}_{0 ; ; ; s}}\left(\epsilon p^{2 a}\right)_{v_{s}}\right],
$$

where $K_{3}=\kappa\left(\lambda, \eta_{\bullet}\right) K^{L} K_{1}^{V} K_{2}^{F_{\text {ext }}} K^{\prime}$, and $K^{\prime}$ is a constant obtained from the bound over the external momenta present in the vertex kernel $\prod_{s=1}^{d} \prod_{v_{s} \in \mathcal{V}_{\bullet} ; s}(\epsilon \tilde{p})_{v_{s}}$; note that $\epsilon$ in (28) is now restricted to internal faces in (29).

Performing the sum over internal momenta $p_{f_{s}}$ must be done in a way to get the lowest possible divergence in (29). This is an optimization procedure that we detail now.

We determine the behavior of some momentum sums. The following results have been detailed in Appendix A. For constants, $B>0, c>0, b>0, a>0$, and $a^{\prime}>0$, and an integer $n \geq 0$, we have

$$
\begin{aligned}
& \sum_{p_{1}, \ldots, p_{D}=1}^{\infty}\left(\sum_{l=1}^{D} p_{l}^{c}\right)^{n} e^{-B \sum_{l=1}^{D}\left(p_{l}^{b}+p_{l}^{a}\right)}=k B^{-\frac{(c n+D)}{b}} e^{-B^{1-\frac{a}{b}}}\left(1+O\left(B^{\frac{1}{b}}\right)\right) \\
& \sum_{p_{1}, \ldots, p_{D}=1}^{\infty}\left(\sum_{l=1}^{D} p_{l}^{c}\right)^{n} e^{-B\left(\sum_{l=1}^{D}\left(p_{l}^{b}+p_{l}^{a}+p_{l}^{a^{\prime}}\right)\right)}=k B^{-\frac{(c n+D)}{b}} e^{-B^{2-\frac{\left(a+a^{\prime}\right)}{b}}}\left(1+O\left(B^{\frac{1}{b}}\right)\right)
\end{aligned}
$$

for a constant $k$. At this point, we make two assumptions on the parameters $a, a^{\prime}, b$ :

- for the model,$+ a \leq b$,

$$
\sum_{p_{1}, \ldots, p_{D}=1}^{\infty}\left(\sum_{l=1}^{D} p_{l}^{c}\right)^{n} e^{-B \sum_{l=1}^{D}\left(p_{l}^{b}+p_{l}^{a}\right)}=k B^{-\frac{(c n+D)}{b}}\left(1+O\left(B^{\frac{1}{b}}\right)+O\left(B^{1-\frac{a}{b}}\right)\right) .
$$

- for the model $\times, a+a^{\prime} \leq 2 b$,

$$
\sum_{p_{1}, \ldots, p_{D}=1}^{\infty}\left(\sum_{l=1}^{D} p_{l}^{c}\right)^{n} e^{-B\left(\sum_{l=1}^{D}\left(p_{l}^{b}+p_{l}^{a}+p_{l}^{a^{\prime}}\right)\right)}=k B^{-\frac{(c n+D)}{b}}\left(1+O\left(B^{\frac{1}{b}}\right)+O\left(B^{2-\frac{\left(a+a^{\prime}\right)}{b}}\right)\right) .
$$

Finally, the integration of internal momenta can be performed in the amplitudes.

\section{B. Model +}

Given a face $f$ (the subscript $s$ is not useful at this stage), we target a line $l_{f}$ such that $i_{l_{f}}=\min _{l \in f} i_{l}=i_{f}$. After the integration, it will generate the lowest factor $M^{i_{f} \times m}$, where $m$ is yet to be determined. 
In the product $\prod_{s=1}^{d} \prod_{v_{s} \in \mathcal{V}_{+; ; ; s}}\left(\epsilon p^{2 a}\right)_{v_{s}}$, we choose the factor of a given $p_{f}$ and perform the sum $\sum_{p_{f}}\left(\left|p_{f}\right|^{2 a}\right)^{\rho_{f}} e^{-\delta M^{-i_{f}}\left|p_{f}\right|^{b}}$, with $\rho_{f}$ an integer, such that the bound (29) still holds. Performing this sum using (32), we get a product of $M^{\frac{i_{f}}{b}}\left(2 a \rho_{f}+D\right)$ with the lowest possible power. Take a closed face $f_{s}$ of color $s$, the integer $\rho_{f_{s}}$ counts how many times $f_{s}$ passes through vertices of $\mathcal{V}_{+; 4 ; s}$. We have

$$
\rho_{f_{s}}=\sum_{v_{s} \in \mathcal{V}_{+; 4 ; s}} \epsilon_{v_{s}, f_{s}}, \quad \rho_{+}(\mathcal{G})=\sum_{s} \sum_{f_{s}} \rho_{f_{s}} .
$$

We then write a new bound

$$
\left|A_{\mathcal{G} ; \boldsymbol{\mu}}\right| \leq \kappa_{1}\left[\prod_{l \in \mathcal{L}} M^{-2 i_{l}}\right] \sum_{p_{f_{s}}}\left[\prod_{f_{s} \in \mathcal{F}_{\text {int }}} e^{-\delta M^{-i f_{s}} \sum_{\xi}\left|p_{f_{s}}\right|^{\xi}}\right]\left[\prod_{s^{\prime}=1}^{d} \prod_{f_{s^{\prime}}}\left|p_{f_{s^{\prime}}}\right|^{2 a \rho_{f_{s^{\prime}}}}\right],
$$

where $\kappa_{1}$ is a new constant incorporating the previous constant $K_{3}$. Performing the sum over internal momenta, one gets using (32) with $a \leq b$,

$$
\left|A_{\mathcal{G} ; \boldsymbol{\mu}}\right| \leq \kappa_{2} \prod_{l \in \mathcal{L}} M^{-2 i_{l}} \prod_{f_{s} \in \mathcal{F}_{\text {int }}} M^{\frac{i_{f_{s}}}{b}\left(2 a \rho_{f_{s}}+D\right)},
$$

where $\kappa_{2}$ is another constant depending on the graph that includes $\kappa_{1}$ and new constants coming from the summation over internal momenta.

We re-express the above bound in terms of the quasi-local subgraphs $G_{k}^{i}$. The product over lines can be written ${ }^{68}$ as

$$
\prod_{l \in \mathcal{L}} M^{-2 i_{l}}=\prod_{l \in \mathcal{L}} \prod_{(i, k) / l \in \mathcal{L}\left(G_{k}^{i}\right)} M^{-2}=\prod_{(i, k)} M^{-2 L\left(G_{k}^{i}\right)} .
$$

The second product over faces splits in two factors. The first term can be treated as

$$
\prod_{f_{s} \in \mathcal{F}_{\text {int }}} M^{\frac{D}{b} i_{f_{s}}}=\prod_{f_{s} \in \mathcal{F}_{\text {int }}} \prod_{(i, k) /} l_{f_{s} \in \mathcal{L}\left(G_{k}^{i}\right)} M^{\frac{D}{b}}=\prod_{(i, k)} \prod_{f_{s} \in \mathcal{F}_{\text {int }} \cap G_{k}^{i}} M^{\frac{D}{b}}=\prod_{(i, k)} M^{\frac{D}{b}} F_{\text {int }}\left(G_{k}^{i}\right) .
$$

The last product involving $\rho_{f_{s}}$ can be treated as

$$
\prod_{f_{s} \in \mathcal{F}_{\text {int }}} \prod_{(i, k) /} M_{l_{f_{s}} \in G_{k}^{i}} M^{\frac{2 a}{b} i_{s}} \rho_{f_{s}}=\prod_{(i, k)} \prod_{f_{s} \in \mathcal{F}_{\text {int }} \cap G_{k}^{i}} M^{\frac{2 a}{b} \rho_{f_{s}}}=\prod_{(i, k)} M^{\frac{2 a}{b} \rho_{+}\left(G_{k}^{i}\right)}
$$

where $\rho_{+}(\cdot)$ has been defined in (34).

Now, if we introduce the counter-term and the wave function vertices $V_{2 ; a ; s}$ and $V_{2 ; b ; s}$, they might bring an additional momentum enhancement to faces. We want to keep the definition of $\rho_{f_{s}}$ as in (34) and we must now add to it the contributions of the 2-point vertices of any types. Hence $\rho_{f_{s}} \rightarrow \rho_{f_{s}}+\rho_{2 ; a ; f_{s}}+\rho_{2 ; b ; f_{s}}$, where $\rho_{2 ; \xi ; f_{s}}=\sum_{v_{s} \in \mathcal{V}_{2 ; \xi ; s}} \epsilon_{v_{s}, f_{s}}$ is the number of times that $f_{s}$ visits $\mathcal{V}_{2 ; \xi ; s}$ vertices, $\xi=a, b$. To the above power counting, we should therefore add the following factor:

$$
\prod_{f_{s} \in \mathcal{F}_{\text {int }}} \prod_{(i, k) / l_{f_{s}} \in G_{k}^{i}} M^{i_{f_{s}}\left[\frac{2 a}{b} \rho_{2 ; a ; f_{s}}+\frac{2 b}{b} \rho_{2 ; b ; f s}\right]}=\prod_{(i, k)} \prod_{f_{s} \in \mathcal{F}_{\text {int }} \cap G_{k}^{i}} M^{\left[\frac{2 a}{b} \rho_{2 ; a ; f_{s}}+2 \rho_{2 ; b ; f s}\right]}
$$

Note that a vertex of $\mathcal{V}_{2 ; \xi ; s}$ has a single strand with an enhanced momentum $p_{s}^{2 \xi}, \xi=a, b$. When a face uses that strand, the corresponding vertex contributes exactly once to the power counting. Then,

$$
\rho_{2 ; \xi}=\sum_{f_{s} \in F_{\text {int }}\left(G_{k}^{i}\right)} \rho_{2 ; \xi ; f_{s}}
$$

counts the number of vertices of $\mathcal{V}_{2 ; \xi ; s}$ in $G_{k}^{i}$. In the end, we have 


$$
\prod_{(i, k)} \prod_{f_{s} \in \mathcal{F}_{\text {int }} \cap G_{k}^{i}} M^{\left[\frac{2 a}{b} \rho_{2 ; a ; f_{s}}+2 \rho_{2 ; b ; f s}\right]}=\prod_{(i, k)} M^{\frac{2 a}{b} \rho_{2 ; a}\left(G_{k}^{i}\right)+2 \rho_{2 ; b}\left(G_{k}^{i}\right)} .
$$

Changing $M \rightarrow M^{b}$, we obtain a power counting of the amplitude (36) for the model + , under the condition $a \leq b$, as

$$
\left|A_{\mathcal{G} ; \boldsymbol{\mu}}\right| \leq \kappa \prod_{(i, k) \subset N^{2}} M^{\omega_{\mathrm{d} ;+}\left(G_{k}^{i}\right)},
$$

where $\kappa$ is a constant and the degree of divergence of $G_{k}^{i}$ is given by

$$
\omega_{\mathrm{d} ;+}\left(G_{k}^{i}\right)=-2 b L\left(G_{k}^{i}\right)+D F_{\mathrm{int}}\left(G_{k}^{i}\right)+2 a \rho_{+}\left(G_{k}^{i}\right)+\sum_{\xi=a, b} 2 \xi \rho_{2 ; \xi}\left(G_{k}^{i}\right) .
$$

Putting $a$ to 0 leads to the ordinary power counting theorem of the usual tensor field theories. ${ }^{47}$ Note that in this Ref. $47, b$ was an arbitrary positive parameter. If $b=1$, one recovers for instance a model like Ref. 40 (although with an interaction of order 6), and for $b=1 / 2$, one finds exactly the power counting of the model of Ref. 42. The above comment holds wherever else in the text. With a quick look on (44), one might think that the degree of divergence of enhanced theories is larger than that of ordinary theories. However, this is not the case: a given graph in a theory without enhanced interactions keeps the same amplitude in the theory with enhanced interactions because it does not receive any enhancement. Therefore, we must only compare amplitudes within the enhanced theory which, in any case, contains them all.

\section{Model $x$}

The analysis is very similar to the above. We count how many times a face $f_{s}$ passes through all vertices of the type $\mathcal{V}_{\times ; s}$ and this defines the following quantities:

$$
\varrho_{f_{s}}=\sum_{v_{s^{\prime}} f_{s^{\prime \prime}}} \epsilon_{v_{s^{\prime}} f_{s_{s}} f_{s^{\prime \prime}}}, \quad \rho_{\times}(\mathcal{G})=\sum_{s} \sum_{f_{s}} \varrho_{f_{s}} .
$$

With a similar calculation as above, using (33) with $3 a \leq 2 b$, introducing also vertices of $\mathcal{V}_{2 ; \xi ; s}$, $\xi=a, 2 a, b$, and $\rho_{2 ; \xi ; f_{s}}$ as the number of times that a closed face $f_{s}$ runs through vertices of $\mathcal{V}_{2 ; \xi ; s}$ and $\rho_{2 ; \xi}$ still obeys (41), we obtain the power counting of the model $\times$ as

$$
\left|A_{\mathcal{G} ; \boldsymbol{\mu}}\right| \leq \kappa \prod_{(i, k) \subset N^{2}} M^{\omega_{\mathrm{d} ; \times}\left(G_{k}^{i}\right)},
$$

where $\kappa$ is a constant and the degree of divergence of $G_{k}^{i}$ is given by

$$
\omega_{\mathrm{d} ; \times}\left(G_{k}^{i}\right)=-2 b L\left(G_{k}^{i}\right)+D F_{\mathrm{int}}\left(G_{k}^{i}\right)+2 a \rho_{\times}\left(G_{k}^{i}\right)+\sum_{\xi=a, 2 a, b} 2 \xi \rho_{2 ; \xi}\left(G_{k}^{i}\right) .
$$

From (44) and (47) and for convenience, we can use unified notations $\omega_{\mathrm{d} ;} \bullet$ with $\bullet=+, \times$, with the sum of $\xi$ being appropriately chosen.

Putting $a$ to 0 reduces to the ordinary power counting theorem of the usual tensor field theories which are, for example, achieved in Ref. 47 where $b$ remains to be treated as an arbitrary positive parameter there as well.

\section{ANALYSES OF THE POTENTIALLY RENORMALIZABLE MODELS}

In this section, we explore the parameter spaces of potentially renormalizable models + and $\times$.

To start, let us define the notion of UV perturbative renormalizability in a model. In generic terms, consider a theory with a given number $n$ of couplings associated with some interactions; the expression for the superficial degree of divergence $\omega_{\mathrm{d}}(\mathcal{G})$, where the terms are proportional to the number $V_{k}$ of vertices of the $k$ th coupling, $k=1, \ldots, n$, of a given graph $\mathcal{G}$, is of the form $\omega_{\mathrm{d}}(\mathcal{G})=\cdots+\sum_{k} c_{k} V_{k}$, and $c_{k}$ is a constant; we say that the model is 
- non-renormalizable if there is a $k$ such that $c_{k}>0$. Amplitudes become more and more divergent as one goes at higher orders in perturbation theory in that the coupling labeled by $k$. For such a $k$ with $c_{k}>0$, the associated coupling is called irrelevant in the IR.

- renormalizable when for all $k, c_{k} \leq 0$. This case further generally splits into two subcases:

(a) if for some $k, c_{k}<0$, the $k$ th coupling is called relevant in the IR. Graph amplitudes become more and more convergent as one goes at higher orders in perturbation theory, in that coupling constant. If all couplings are such that $c_{k}<0$, then only a finite number of graph amplitudes are divergent and will contribute to the flow: then the model is called super-renormalizable.

(b) If $c_{k}=0$, for some $k$, the $k$ th coupling is called marginal. The divergence degree $\omega_{d}(\mathcal{G})$ is independent of the order of perturbation in that vertex $k$. An infinite number of graphs might be divergent. If, in particular, $c_{k}=0$ for $k=k_{\max }$, for the highest order valence among the interactions, then the model is called just-renormalizable. For this model, we usually expect that the $k_{\max }$-point amplitudes are exactly logarithmically divergent.

To fix the ideas, consider the ordinary $\phi^{4}$ in 4D: the interactions are the wave function, the mass, and the coupling $\phi^{4}$. We have $\sum_{k} c_{k} V_{k}=(0) V_{2}^{\prime}+(-2) V_{2}+(0) V_{4}=-2 V_{2}, V_{2}^{\prime}$ is the number of wave function vertices, $V_{2}$ mass vertices, and $V_{4}$ the number of $\phi^{4}$ vertices. The model is just renormalizable, and the mass and the $\phi_{4}$ coupling are called relevant in the IR and marginal, respectively.

In standard QFT, the constant $\left(-c_{k}\right)$ corresponds to the scaling dimension of the coupling which also coincides with the canonical dimension obtained from the classical action (see Appendix $\mathrm{C}$ in Ref. 33). However, in nonlocal models like matrix and tensor field theory, the scaling dimension of a coupling bears a particular definition (see again the above reference): this is the maximum of the divergence degree on all graph amplitudes with boundary data reproducing the nonlocal pattern of that coupling. We can prove also that, specifically for couplings corresponding to melonic interactions, $\left(-c_{k}\right)$ is again the scaling dimension. In the same vein, the question of the scaling dimensions of couplings associated with enhanced interactions can be addressed. We can use, in principle, the same definition of the scaling dimension of a coupling in the usual tensor field theory (as the maximum of the divergence degree) in the present case. However, at the technical level, the treatment of the divergence degree will be modified due to the combinatorial quantities, namely, $\rho_{\bullet}$, introduced by the enhancement. At this stage, we could only find an upper bound (which however saturates) on the divergence degree $\omega_{\mathrm{d}}(\mathcal{G}) \leq \cdots+\sum_{k} c_{k} V_{k}$, and from that point perform the usual analysis. As a consequence, as we will see in the following, in some cases the meaning of a relevant and marginal coupling should be handled with a great care. Specifically, this inequality for the model + leads to the usual conclusion, meanwhile for the model $\times$, it leads to a discrepancy with the meaning of ordinary just-renormalizable theory. This treatment is the object of Secs. V A-VII.

In the analyses below, we need the number of internal faces of a connected graph $\mathcal{G}$, in any rank $d \geq 3$ tensorial model, which is given in Ref. 45

$$
F_{\text {int }}=-\frac{2}{\left(d^{-}\right) !}\left(\omega\left(\mathcal{G}_{\text {color }}\right)-\omega(\partial \mathcal{G})\right)-\left(C_{\partial \mathcal{G}}-1\right)-\frac{d^{-}}{2} N_{\text {ext }}+d^{-}-\frac{d^{-}}{4}(4-2 n) \cdot V,
$$

where $d^{-}=d-1$ with $d$ being the rank of the tensor field, $\mathcal{G}_{\text {color }}$ is the colored extension of $\mathcal{G}$, $\partial \mathcal{G}$ denotes the boundary of $\mathcal{G},{ }^{40}$ with $C_{\partial \mathcal{G}}$ the number of connected components of $\partial \mathcal{G}, N_{\text {ext }}$ is the number of external legs of the graph, $V_{k}$ is the number of vertices of coordination number $k$, $V=\sum_{k} V_{k}$ is the total number of vertices in $\mathcal{G}$, and $n \cdot V=\sum_{k} k V_{k}$ is the number of half lines emanating from vertices. $\omega\left(\mathcal{G}_{\text {color }}\right)=\sum_{J_{\mathcal{G}_{\text {color }}}} g_{\widetilde{J}_{\mathcal{G}_{\text {color }}}}, \omega(\partial \mathcal{G})=\sum_{J_{\partial \mathcal{G}}} g_{J_{\partial \mathcal{G}}}$ with genus $g_{J}$, the genus of a ribbon graph $J$ called jacket. ${ }^{13}$ A jacket is nothing but a particular embedding of the bipartite colored graph $\mathcal{G}$. The jackets of $\mathcal{G}_{\text {color }}$ are denoted $J_{\mathcal{G}_{\text {color }}}$ and they must be "closed" to define a closed surface $\widetilde{J}_{\mathcal{G}_{\text {color }}}$ on which a genus $g_{\widetilde{J}_{\mathcal{G}_{\text {color }}}}$ could be identified. The boundary graph $\partial \mathcal{G}$ itself maps to a rank $d-1$ colored tensor graph. $\partial \mathcal{G}$ therefore has jackets denoted $J_{\partial \mathcal{G}}$. 
The quantity $\omega\left(\mathcal{G}_{\text {color }}\right)$ is called the degree of the colored tensor graph $\mathcal{G}_{\text {color }}$. It replaces the genus and allows one to define a large $N$ expansion for colored tensor models. ${ }^{13}$ A graph $\mathcal{G}$ is called a melon if and only if its colored extension $\mathcal{G}_{\text {color }}$ is a melon and that is if $\omega\left(\mathcal{G}_{\text {color }}\right)=0$ (all jackets $\widetilde{J}_{\mathcal{G}_{\text {color }}}$ are planar). We shall need a few properties of the quantity $\omega\left(\mathcal{G}_{\text {color }}\right)-\omega(\partial \mathcal{G})$ withdrawn from Ref. 41 that we will recall at some point.

Let us recall the following terminology: a "bridge" in a graph is a line such that cutting that line adds another connected component to this graph. The "cut of a bridge" means the removal of the bridge from the graph and letting two external legs where its extremities were incident. A graph is called one-particle reducible (1PR) graph if it has bridges; otherwise it is called one-particle irreducible (1PI).

Lemma 1 ( $\rho \bullet$ and $\rho 2 ; \xi$ for $1 \mathrm{PR}$ graph). Let $\mathcal{G}$ be a graph with bridges (or a 1PR graph) such that cutting the bridges gives the family $\left\{\mathcal{G}_{j}\right\}$ of subgraphs. Then

$$
\rho_{\bullet}(\mathcal{G})=\sum_{j} \rho_{\bullet}\left(\mathcal{G}_{j}\right), \quad \rho_{2 ; \xi}(\mathcal{G})=\sum_{j} \rho_{2 ; \xi}\left(\mathcal{G}_{j}\right),
$$

where $\bullet=+$, and $\xi=a, b$, or $\bullet=\times$, and $\xi=a, 2 a, b$.

Proof. This follows from the fact that through a bridge, no closed face passes. The quantities $\rho_{\bullet}(\mathcal{G})$ and $\rho_{2 ; \xi}(\mathcal{G})$ can be computed with the block diagonal matrix $\epsilon_{v f}$ using vertices and closed faces in each connected component $\mathcal{G}_{j}$.

The following proposition is easy to prove.

Lemma 2 (Bounds on $\rho_{2 ; \xi}$ ). Let $\mathcal{G}$ be a graph of the model $\bullet$. Then $\rho_{2 ; \xi}(\mathcal{G}) \leq V_{2 ; \xi}$. If $\mathcal{G}$ is 1 PI then

$$
\rho_{2 ; \xi}(\mathcal{G})=V_{2 ; \xi}(\mathcal{G})
$$

\section{A. Models +}

Consider the "contraction" operation of a degree-2 vertex $v$ (belonging to $\mathcal{V}_{2}$ or to $\mathcal{V}_{2 ; s}$ ) on the graph $\mathcal{G}$ which removes $v$ and replaces it by a propagator line with the same external momenta. Consider the graph $\tilde{\mathcal{G}}$ resulting from the contractions of all degree-2 vertices in $\mathcal{G}$. Note that if $\mathcal{G}$ is $1 \mathrm{PR}$ or 1PI, then so is $\tilde{\mathcal{G}}$ and the number of degree- 4 vertices and external legs coincide in both graphs. We define the number $\mathrm{Br}$ of c-bridges (chain-bridges) of $\mathcal{G}$ to be the number of bridges in $\tilde{\mathcal{G}}$. Note that a c-bridge of $\mathcal{G}$ can be very well associated with a bridge of $\mathcal{G}$. We also introduce $V_{4}+V_{+; 4}=V_{(4)}$.

Lemma 3 (Bound of $\rho+$ ). Let $\mathcal{G}$ be a graph with $N_{\mathrm{ext}}>0$ external legs. Then, $\rho_{+}(\mathcal{G}) \leq V_{+; 4}$. If $\mathcal{G}$ is melonic

- $V_{(4)}=1$, then $\rho_{+}(\mathcal{G})=0$,

- $V_{(4)}>1$, then $\rho_{+}(\mathcal{G}) \leq V_{(4)}-\frac{N_{\text {ext }}}{2}-B r$,

where $\mathrm{Br}$ is the number of $c$-bridges in the graph $\mathcal{G}$.

Proof. The first statement is clear from the combinatorial procedure counting at most $V_{+; 4}$ for $\rho_{+}(\mathcal{G})$ for an arbitrary graph. Now this bound can be refined for a melonic $N_{\text {ext }}$-point graph. If $V_{(4)}=1$, then either $N_{\text {ext }}=4$, and then $\rho_{+}(\mathcal{G})=0$, or $N_{\text {ext }}=2$, and we have a melonic tadpole or a melonic graph with one c-bridge which gives again $\rho_{+}(\mathcal{G})=0$.

A 1 PI graph $\mathcal{G}$ with 4 valent vertices can have at most 2 external legs per vertex. Consider a melonic graph $\mathcal{G}$ and its colored extension $\mathcal{G}_{\text {color }}$ : then each vertex in $\mathcal{G}_{\text {color }}$ comes with a partner (see for instance Fig. 1 in Ref. 41). Note that the two partner vertices belong to the same vertex in $\mathcal{G}$. If one vertex $v$ has a propagator $l$ and its partner $\tilde{v}$ has no propagator (hence has an external leg), then $l$ must be a bridge. Focusing on 1PI bipartite melons, then either $v$ and $\tilde{v}$ have both propagators or have both external legs. The presence of $N_{\text {ext }}$ external legs in 1PI bipartite melons implies that these external legs must be hooked to $N_{\text {ext }} / 2$ vertices. Take any vertex $v_{s}$ with color $s$ where an external leg 
is incident, then an external leg is also incident to $\tilde{v}_{s}$. None of the open faces with color $0 s$, which can be enhanced, could bring any contribution to $\rho_{+}(\mathcal{G})$. Repeating the argument for $N_{\text {ext }} / 2$ vertices, we see that these vertices could not be part of the optimization procedure computing $\rho_{+}(\mathcal{G})$ and so $\rho_{+}(\mathcal{G}) \leq V_{(4)}-N_{\text {ext }} / 2$.

Now we treat the case of a $1 P R$ graph $\mathcal{G}$. Consider its resulting $\tilde{\mathcal{G}}$ after the contraction of all of its degree-2 vertices. Cut all bridges in $\tilde{\mathcal{G}}$ to obtain a family of 1PI subgraphs. On each component $\tilde{\mathcal{G}}_{j}$, the bound $\rho_{+}\left(\tilde{\mathcal{G}}_{j}\right) \leq V_{(4)}\left(\tilde{\mathcal{G}}_{j}\right)-\frac{N_{\text {ext }}\left(\tilde{\mathcal{G}}_{j}\right)}{2}$ holds. Summing this relation over 1PI subgraphs and using Lemma 1 , and using the relation $\rho_{+}(\mathcal{G})=\rho_{+}(\tilde{\mathcal{G}})$ because degree- 2 vertices are not involved in the counting of $\rho_{+}$and therefore the total number of bridges is simply $B r$, we get

$$
\rho_{+}(\tilde{\mathcal{G}})=\sum_{j} \rho_{+}\left(\tilde{\mathcal{G}}_{j}\right) \leq \sum_{j}\left[V_{(4)}\left(\tilde{\mathcal{G}}_{j}\right)-\frac{N_{\text {ext }}\left(\tilde{\mathcal{G}}_{j}\right)}{2}\right]=V_{(4)}-\frac{1}{2} N_{\text {ext }}-B r,
$$

where we used that each bridge cut brings two additional external legs compared to $N_{\text {ext }}$. In summary, we can also use (51) for 1PI graph with $B r=0$.

As an illustration of Lemma 3, consider the graphs of Fig. 4. Consider the melonic graph at the left-hand side. $\frac{N_{\text {ext }}}{2}=3$ vertices which have external legs will not contribute to $\rho_{+}(\mathcal{G})$. Hence, $\rho_{+}(\mathcal{G}) \leq V_{(4)}-\frac{N_{\text {ext }}}{2}$. On the other hand, consider the non-melonic graph on the right-hand side. 3 vertices which have external legs contribute to $\rho_{+}(\mathcal{G})$.

For a melonic graph, Lemma 3 gives in fact two bounds. The bound $\rho_{+}(\mathcal{G}) \leq V_{+; 4}$ is sharper than the other, if and only if

$$
V_{4} \geq \frac{N_{\text {ext }}}{2}+B r
$$

\section{Potentially renormalizable models}

We restrict now to primitively divergent graphs which can be considered connected and with $B r=0$, in other words to 1PI graphs. The degree of divergence of this model is, by combining (44) and (48) and using $2 L=n \cdot V-N_{\mathrm{ext}}, a \leq b$,

$$
\begin{aligned}
\omega_{\mathrm{d} ;+}(\mathcal{G})= & -\frac{2 D}{\left(d^{-}\right) !}\left(\omega\left(\mathcal{G}_{\text {color }}\right)-\omega(\partial \mathcal{G})\right)-D\left(C_{\partial \mathcal{G}}-1\right)-\frac{1}{2}\left[\left(D d^{-}-2 b\right) N_{\mathrm{ext}}-2 D d^{-}\right] \\
& +\frac{1}{2}\left[-2 D d^{-}+\left(D d^{-}-2 b\right) n\right] \cdot V+2 a \rho_{+}+2 a \rho_{2 ; a}+2 b \rho_{2 ; b} .
\end{aligned}
$$

From Lemma 3, we have

$$
\begin{aligned}
\Delta_{+}^{\text {melon }} & = \begin{cases}0, & V_{(4)}=1, \\
V_{(4)}-\frac{N_{\mathrm{ext}}}{2}-\rho_{+}\left(\mathcal{G}^{\text {melon }}\right) \geq 0, & V_{(4)}>1,\end{cases} \\
\Delta_{+} & =V_{+; 4}-\rho_{+}(\mathcal{G}) \geq 0 .
\end{aligned}
$$

The case $V_{(4)}>1$ is the most important one when we study all orders of perturbation and we will focus on that. Using Lemma 2, and further inserting that $\omega\left(\mathcal{G}_{\text {color }}\right)=0$ and $\omega(\partial \mathcal{G})=0$ for melonic graphs,
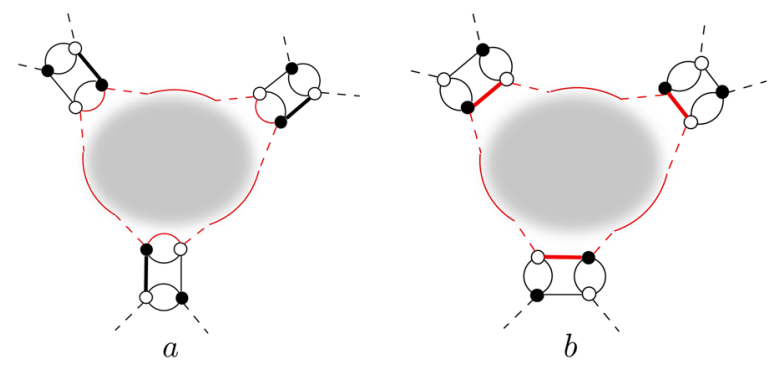

FIG. 4. Examples of $N_{\mathrm{ext}}=6$-point functions in rank $d=3$ of a melonic type and a non-melonic type. 


$$
\begin{aligned}
& \omega_{\mathrm{d} ;++}\left(\mathcal{G}^{\text {melon }}\right) \leq-D\left(C_{\partial \mathcal{G}}-1\right)-\frac{1}{2}\left[\left(D d^{-}-2 b+2 a\right) N_{\mathrm{ext}}-2 D d^{-}\right] \\
& -2 b V_{2}-2(b-a) V_{2 ; a}+\left(D d^{-}-4 b+2 a\right) V_{(4)}-2 a \triangle_{+}^{\mathrm{melon}} \\
& \leq-D\left(C_{\partial \mathcal{G}}-1\right)-\frac{1}{2}\left[\left(D d^{-}-2 b+2 a\right) N_{\mathrm{ext}}-2 D d^{-}\right] \\
& -2 b V_{2}-2(b-a) V_{2 ; a}+\left(D d^{-}-4 b+2 a\right) V_{(4)} .
\end{aligned}
$$

There is another bound for melonic graphs

$$
\begin{aligned}
& \omega_{\mathrm{d} ;+}\left(\mathcal{G}^{\text {melon }}\right) \leq-D\left(C_{\partial \mathcal{G}}-1\right)-\frac{1}{2}\left[\left(D d^{-}-2 b\right) N_{\mathrm{ext}}-2 D d^{-}\right] \\
& -2 b V_{2}-2(b-a) V_{2 ; a}+\left(D d^{-}-4 b\right) V_{4}+\left(D d^{-}-4 b+2 a\right) V_{+; 4} \\
& \leq-D\left(C_{\partial \mathcal{G}}-1\right)-\left[b N_{\mathrm{ext}}-D d^{-}\right] \\
& -2 b V_{2}-2(b-a) V_{2 ; a}+\left(D d^{-}-4 b\right)\left(V_{4}-\frac{N_{\mathrm{ext}}}{2}\right)+\left(D d^{-}-4 b+2 a\right) V_{+; 4} .
\end{aligned}
$$

Either choosing (56) or (55) as a sharper bound leads to the same result.

Meanwhile, for non-melonic graphs, using $\omega\left(\mathcal{G}_{\text {color }}\right)-\omega(\partial \mathcal{G}) \geq \frac{1}{2}\left(d^{-}-1\right) d^{-}$!, ${ }^{41}$ we get

$$
\begin{aligned}
& \omega_{\mathrm{d} ;+}\left(\mathcal{G}^{\text {non-melon }}\right) \leq-D\left(d^{-}-1\right)-D\left(C_{\partial \mathcal{G}}-1\right)-\frac{1}{2}\left[\left(D d^{-}-2 b\right) N_{\mathrm{ext}}-2 D d^{-}\right] \\
& -2 b V_{2}-2(b-a) V_{2 ; a}+\left(D d^{-}-4 b\right) V_{4}+\left(D d^{-}-4 b+2 a\right) V_{+; 4}-2 a \triangle_{+} \\
& \leq-D\left(d^{-}-1\right)-D\left(C_{\partial \mathcal{G}}-1\right)-\frac{1}{2}\left[\left(D d^{-}-2 b\right) N_{\mathrm{ext}}-2 D d^{-}\right] \\
& -2 b V_{2}-2(b-a) V_{2 ; a}+\left(D d^{-}-4 b\right) V_{4}+\left(D d^{-}-4 b+2 a\right) V_{+; 4} .
\end{aligned}
$$

For renormalizable models, we require the coefficients of vertices to be negative. This is demanding since $a>0, b>0$,

$$
D d^{-}-4 b+2 a \leq 0, \quad b \geq a,
$$

which give for $a$,

$$
a \leq 2 b-\frac{1}{2} D d^{-}
$$

We see that the condition $a \leq b$ coming from the sum over internal momenta has been naturally incorporated in the analysis. Then, to achieve just-renormalizability, we use $a=2 b-\frac{1}{2} D d^{-} \geq 0$ (and $a \leq b$ implies that $b \leq \frac{1}{2} D d^{-}$) given in (59) into (55) and (57), and see if the conditions

$$
\left.\omega_{\mathrm{d} ;+}\left(\mathcal{G}^{\text {melon }}\right)\right|_{N_{\text {ext }} \geq 6}<0,\left.\quad \omega_{\mathrm{d} ;+}\left(\mathcal{G}^{\text {non-melon }}\right)\right|_{N_{\text {ext }} \geq 6}<0
$$

can be accomplished. These conditions translate into

$$
\begin{aligned}
& \left.\omega_{\mathrm{d} ;+}\left(\mathcal{G}^{\text {melon }}\right)\right|_{N_{\mathrm{ext}} \geq 6} \leq \\
& {\left.\left[-D\left(C_{\partial \mathcal{G}}-1\right)+D d^{-}-b N_{\mathrm{ext}}-2 b V_{2}-2(b-a) V_{2 ; a}\right]\right|_{N_{\mathrm{ext}} \geq 6}<0} \\
& \left.\omega_{\mathrm{d} ;+}\left(\mathcal{G}^{\text {non-melon }}\right)\right|_{N_{\mathrm{ext}} \geq 6} \leq \\
& {\left.\left[D-D\left(C_{\partial \mathcal{G}}-1\right)-\left(\frac{1}{2} D d^{-}-b\right) N_{\mathrm{ext}}-2 b V_{2}-2(b-a) V_{2 ; a}-2 a V_{4}\right]\right|_{N_{\mathrm{ext}} \geq 6}<0}
\end{aligned}
$$

As $N_{\text {ext }}$ increases, $\omega_{\mathrm{d} ;+}$ decreases, so $\omega_{\mathrm{d} ;+}$ is maximum at $N_{\text {ext }}=6$ for melonic graphs; $\omega_{\mathrm{d} ;+}$ is maximum at $N_{\text {ext }}=6$ as long as $b<\frac{D d^{-}}{2}$, for non-melonic graphs. Thus, the conditions for having convergent $N_{\text {ext }}=6$-point functions are

$$
\begin{aligned}
\left.\omega_{\mathrm{d} ;+}\left(\mathcal{G}^{\text {melon }}\right)\right|_{N_{\mathrm{ext}}=6} & \leq-D\left(C_{\partial \mathcal{G}}-1\right)+D d^{-}-6 b-2 b V_{2}-2(b-a) V_{2 ; a} \\
& \leq D d^{-}-6 b<0, \\
\left.\omega_{\mathrm{d} ;+}\left(\mathcal{G}^{\text {non-melon }}\right)\right|_{N_{\mathrm{ext}}=6} & \leq D-D\left(C_{\partial \mathcal{G}}-1\right)-3 D d^{-}+6 b-2 b V_{2}-2(b-a) V_{2 ; a}-2 a V_{4} \\
& \leq D-3 D d^{-}+6 b<0 .
\end{aligned}
$$


The above inequalities further reduce to

$$
\frac{D d^{-}}{6}<b<\frac{D\left(3 d^{-}-1\right)}{6}
$$

Note here that $\frac{D\left(3 d^{-}-1\right)}{6}<\frac{D d^{-}}{2}$ is always true for $D>0$, thus we have improved the bound on $b$. Under (65), the degree of divergence for $N_{\text {ext }} \geq 6$ is maximum at $N_{\text {ext }}=6$ and strictly negative. Furthermore, we demand that $a>0$ and so that $b>\frac{D d^{-}}{4}$. We finally get the bound

$$
\frac{D d^{-}}{4}<b<\frac{D\left(3 d^{-}-1\right)}{6}
$$

Now we use (66) to find a bound on $a=2 b-\frac{1}{2} D d^{-}$(59) as

$$
0<a<\frac{D\left(3 d^{-}-2\right)}{6}
$$

Combining (67) and (66) for given $D$ and $d^{-} \geq 2$, we obtain the ranges of values of $a$ and $b$ in Table I which could lead to just-renormalizable models. This table shows that there might be uncountably many models which could be just renormalizable. We note that the limit cases $a=0$ lead to the renormalizable invariant tensor field theories studied in Ref. $42\left(d=3, D=1, b=\frac{1}{2}\right)$ and Ref. 47 $\left[\left(d=4, D=1, b=\frac{3}{4}\right) ;(d=5, D=1, b=1) ;(d=3, D=2, b=1)\right]$.

Let us seek further conditions leading to interesting models with $a>0$. One of these conditions is to achieve logarithmic divergence for non-melonic graphs at $N_{\text {ext }}=4$. For this, achieving

$$
\left.\omega_{\mathrm{d} ;+}\left(\mathcal{G}^{\text {non-melon }}\right)\right|_{N_{\text {ext }}=4}=0
$$

entails

$$
b=\frac{1}{2} D\left(d^{-}-\frac{1}{2}\right), \quad a=\frac{1}{2} D\left(d^{-}-1\right),
$$

which is consistent with (66) since $\frac{1}{2} D\left(d^{-}-\frac{1}{2}\right)<\frac{D\left(3 d^{-}-1\right)}{6}$ for $D>0$ and $\frac{D d^{-}}{4}<\frac{1}{2} D\left(d^{-}-\frac{1}{2}\right)$ for $d^{-}>1$. In Table II, we explicitly show the valid values of $a$ and $b$ given in (69).

Tables I and II are consistent for just-renormalizable models with the superficial degree of

\begin{tabular}{|c|c|c|c|c|}
\hline & $d^{-}=2$ & $d^{-}=3$ & $d^{-}=4$ & $d^{-}=5$ \\
\hline$D=1$ & $\begin{array}{l}0<a<\frac{2}{3} \\
\frac{1}{2}<b<\frac{5}{6}\end{array}$ & $\begin{array}{l}0<a<\frac{7}{6} \\
\frac{3}{4}<b<\frac{4}{3}\end{array}$ & $\begin{array}{l}0<a<\frac{5}{3} \\
1<b<\frac{11}{6}\end{array}$ & $\begin{array}{l}0<a<\frac{13}{6} \\
\frac{5}{4}<b<\frac{7}{3}\end{array}$ \\
\hline$D=2$ & $\begin{array}{l}0<a<\frac{4}{3} \\
1<b<\frac{5}{3}\end{array}$ & $\begin{array}{l}0<a<\frac{7}{3} \\
\frac{3}{2}<b<\frac{8}{3}\end{array}$ & $\begin{array}{l}0<a<\frac{10}{3} \\
2<b<\frac{11}{3}\end{array}$ & $\begin{array}{l}0<a<\frac{13}{3} \\
\frac{5}{2}<b<\frac{14}{3}\end{array}$ \\
\hline$D=3$ & $\begin{array}{l}0<a<2 \\
\frac{3}{2}<b<\frac{5}{2}\end{array}$ & $\begin{array}{l}0<a<\frac{7}{2} \\
\frac{9}{4}<b<4\end{array}$ & $\begin{array}{l}0<a<5 \\
3<b<\frac{11}{2}\end{array}$ & $\begin{array}{l}0<a<\frac{13}{2} \\
\frac{15}{4}<b<7\end{array}$ \\
\hline$D=4$ & $\begin{array}{c}0<a<\frac{8}{3} \\
2<b<\frac{10}{3}\end{array}$ & $\begin{array}{l}0<a<\frac{14}{3} \\
3<b<\frac{16}{3}\end{array}$ & $\begin{array}{l}0<a<\frac{20}{3} \\
4<b<\frac{22}{3}\end{array}$ & $\begin{array}{l}0<a<\frac{26}{3} \\
5<b<\frac{28}{3}\end{array}$ \\
\hline
\end{tabular}
divergence which does not depend on $V_{4}$, with logarithmic divergence for graphs with $N_{\text {ext }}=4$, and with convergent graphs with $N_{\mathrm{ext}} \geq 6$. Let us discuss the behavior of melonic graphs. Concentrating on $N_{\text {ext }}=4$, we evaluate $\left.\omega_{\mathrm{d} ;+}\left(\mathcal{G}^{\text {melon }}\right)\right|_{N_{\text {ext }}=4}$ keeping in mind (66) and obtain

TABLE I. Allowed region of the values of $a$ and $b$ for potentially just-renormalizable models + with $d^{-} \leq 5$ and $D \leq 4$. 
TABLE II. Values of $a$ and $b$ for potentially just-renormalizable theories with $\left.\omega_{\mathrm{d} ;+}\left(\mathcal{G}^{\text {non-melon }}\right)\right|_{N_{\mathrm{ext}}=4}=0$ with $d^{-} \leq 5$ and $D \leq 4$.

\begin{tabular}{|c|c|c|c|c|}
\hline & $d^{-}=2$ & $d^{-}=3$ & $d^{-}=4$ & $d^{-}=5$ \\
\hline$D=1$ & $\begin{array}{l}a=\frac{1}{2} \\
b=\frac{3}{4}\end{array}$ & $\begin{array}{l}a=1 \\
b=\frac{5}{4}\end{array}$ & $\begin{array}{l}a=\frac{3}{2} \\
b=\frac{7}{4}\end{array}$ & $\begin{array}{l}a=2 \\
b=\frac{9}{4}\end{array}$ \\
\hline$D=2$ & $\begin{array}{l}a=1 \\
b=\frac{3}{2}\end{array}$ & $\begin{array}{l}a=2 \\
b=\frac{5}{2}\end{array}$ & $\begin{array}{l}a=3 \\
b=\frac{7}{2}\end{array}$ & $\begin{array}{l}a=4 \\
b=\frac{9}{2}\end{array}$ \\
\hline$D=3$ & $\begin{array}{l}a=\frac{3}{2} \\
b=\frac{9}{4}\end{array}$ & $\begin{array}{c}a=3 \\
b=\frac{15}{4}\end{array}$ & $\begin{array}{c}a=\frac{9}{2} \\
b=\frac{21}{4}\end{array}$ & $\begin{array}{c}a=6 \\
b=\frac{27}{4}\end{array}$ \\
\hline$D=4$ & $\begin{array}{l}a=2 \\
b=3\end{array}$ & $\begin{array}{l}a=4 \\
b=5\end{array}$ & $\begin{array}{l}a=6 \\
b=7\end{array}$ & $\begin{array}{l}a=8 \\
b=9\end{array}$ \\
\hline
\end{tabular}

$$
\left.\omega_{\mathrm{d} ;+}\left(\mathcal{G}^{\text {melon }}\right)\right|_{N_{\mathrm{ext}}=4} \leq D d^{-}-4 b,
$$

which gives

$$
\left.\omega_{\mathrm{d} ;+}\left(\mathcal{G}^{\text {melon }}\right)\right|_{N_{\mathrm{ext}}=4}<0 .
$$

Therefore, we have convergent melonic graphs at $N_{\text {ext }}=4$. Divergent non-melonic graphs at $N_{\text {ext }}=4$ dominate all melonic graphs.

Insisting on having a derivative coupling in the direct space, that is, on $\left(U(1)^{D}\right)^{d}$, we impose that $a$ and $b$ are integers. In that situation, we have the obvious solutions to make $D$ a multiple of 4 . Having covered the parameter space for finding interesting models, we will prove that, in Sec. VI, all models for generic $(d, D)$ (including those of Table II) are in fact just-renormalizable.

\section{B. Models $\times$}

We work under the same definition and conditions as in Sec. V A, where $V_{(4)}$ presently denotes $V_{4}+V_{\times ; 4}$

Lemma 4 (Bound of $\rho_{\times}$). Let $\mathcal{G}$ be a graph with $N_{\mathrm{ext}}$ external legs and Br c-bridges. We have $\rho_{\times}(\mathcal{G}) \leq 2 V_{\times ; 4}$.

If $\mathcal{G}$ is such that

(a) $V_{(4)}=1$ and $N_{\text {ext }}=4$, then $\rho_{\times}(\mathcal{G})=0$,

(b) $V_{(4)}=1, N_{\mathrm{ext}}=2, \rho_{\times}(\mathcal{G}) \leq 1$, and

(c) $V_{(4)}>1$, then $\rho_{\times}(\mathcal{G}) \leq 2 V_{(4)}-\frac{N_{\text {ext }}}{2}-B r$.

If $\mathcal{G}$ is melonic and

(d) $V_{(4)}=1, N_{\text {ext }}=2$ then $\rho_{\times}(\mathcal{G})=0$ and

(e) $V_{(4)}>1$, then $\rho_{\times}(\mathcal{G}) \leq 2 V_{(4)}-N_{\text {ext }}-2 B r$.

Proof. The first statement should not bring any difficulties. Now let us consider a general 1PI graph. Assume $V_{(4)}=1$ and $N_{\text {ext }}=4$, then $\rho_{\times}(\mathcal{G})=0$, there are no closed faces, and so nothing to count. Now if $V_{(4)}=1, N_{\mathrm{ext}}=2$, two cases might happen. Either the graph is melonic, and there are no enhanced faces, i.e., $\rho_{\times}(\mathcal{G})=0$, or the graph is non-melonic and the vertex might still contribute or not to $\rho_{\times}(\mathcal{G})$, thus $\rho_{\times}(\mathcal{G}) \leq 1$. Then we have shown that a, b, and d, are true for any 1PI graphs. For 1PR graph, we simply observe that the presence of bridge at the external legs will not affect the counting of internal faces visiting the vertex counting in $V_{(4)}$. Thus $a, b$, and $d$ are valid in this case.

A 1PI graph, with $V_{(4)}>1$, has at most 2 external legs per vertex. Consider a vertex having exactly 1 external leg: then this vertex will contribute at most 1 to $\rho_{\times}$. If a vertex has 2 external legs, then 2 cases may occur: either the 2 legs are on the same external face which cannot contribute to $\rho_{\times}$ 
or the legs are incident to partner vertices. In the latter case, there are 2 external faces of that vertex which cannot contribute to $\rho_{\times}$. Hence, the upper bound for $\rho_{\times}(\mathcal{G})$ is $2 V_{(4)}-N_{\text {ext }} / 2$.

For a 1PR graph $\mathcal{G}$, we cut all bridges to obtain 1PI subgraphs of the graph $\tilde{\mathcal{G}}$. On each component $\tilde{\mathcal{G}}_{j}$, we use the 1PI general bound $\rho_{\times}\left(\tilde{\mathcal{G}}_{j}\right) \leq 2 V_{(4)}\left(\tilde{\mathcal{G}}_{j}\right)-\frac{1}{2} N_{\text {ext }}\left(\tilde{\mathcal{G}}_{j}\right)$. As we perform in the Proof of Lemma 3 , we can show that the sum over the components brings $\rho_{\times}(\mathcal{G})=\rho_{\times}(\tilde{\mathcal{G}}) \leq 2 V_{(4)}-\frac{N_{\text {ext }}}{2}-B r$.

For a melonic graph, the above bounds must be refined. According to the same discussion in the Proof of Lemma 3, we know that for a 1PI melonic graph, each vertex having external legs must have $N_{\text {ext }}=2$. (If $N_{\text {ext }}=4$, then the vertex gets disconnected and this is the case with $V_{(4)}=1$.) These two external legs must be on partner vertices $v$ and $\tilde{v}$. Hence the enhanced faces on this vertex are necessarily external and cannot contribute to $\rho_{\times}(\mathcal{G})$. Repeating the argument for all vertices with external legs, we get $\rho_{\times}(\mathcal{G}) \leq 2\left(V_{(4)}-\frac{N_{\text {ext }}}{2}\right)=2 V_{(4)}-N_{\text {ext }}$.

Consider a melonic 1 PR graph $\mathcal{G}$. Using again the same strategy, we cut all the bridges in $\tilde{\mathcal{G}}$, and applying the relation $\rho_{\times}\left(\tilde{\mathcal{G}}_{j}\right) \leq 2 V_{(4)}\left(\tilde{\mathcal{G}}_{j}\right)-N_{\text {ext }}\left(\tilde{\mathcal{G}}_{j}\right)$ for each 1PI component, we get

$$
\rho_{\times}\left(\tilde{\mathcal{G}}^{\text {melon }}\right)=\sum_{j} \rho_{\times}\left(\tilde{\mathcal{G}}_{j}\right) \leq \sum_{j}\left[2 V_{(4)}\left(\tilde{\mathcal{G}}_{j}\right)-N_{\text {ext }}\left(\tilde{\mathcal{G}}_{j}\right)\right]=2 V_{(4)}-N_{\text {ext }}-2 B r,
$$

which together with $\rho_{\times}\left(\mathcal{G}^{\text {melon }}\right)=\rho_{\times}\left(\tilde{\mathcal{G}}^{\text {melon }}\right)$ is the second relation for melonic graphs for $V_{(4)}>1$.

The bounds of Lemma 4 should be chosen wisely when bounding the degree of divergence of the graph. Furthermore, again the generic case of $V_{(4)}>1$ will be the important one that we will concentrate on.

\section{Potentially renormalizable models}

We study only primitively divergent graphs and fix $B r=0$. Combining (47) and (48) and using $2 L=n \cdot V-N_{\text {ext }}$, we obtain the bound for the degree of divergence in this model, at $3 a \leq 2 b$,

$$
\begin{aligned}
\omega_{\mathrm{d} ; \times}(\mathcal{G})= & -\frac{2 D}{\left(d^{-}\right) !}\left(\omega\left(\mathcal{G}_{\text {color }}\right)-\omega(\partial \mathcal{G})\right)-D\left(C_{\partial \mathcal{G}}-1\right)-\frac{1}{2}\left[\left(D d^{-}-2 b\right) N_{\mathrm{ext}}-2 D d^{-}\right] \\
& +\frac{1}{2}\left[-2 D d^{-}+\left(D d^{-}-2 b\right) n\right] \cdot V+2 a \rho_{\times}+\sum_{\xi=a, 2 a, b} 2 \xi \rho_{2 ; \xi} .
\end{aligned}
$$

Using Lemma 4 , and further inserting that $\omega\left(\mathcal{G}_{\text {color }}\right)=0$ and $\omega(\partial \mathcal{G})=0$ for melonic graphs, and $\omega\left(\mathcal{G}_{\text {color }}\right)-\omega(\partial \mathcal{G}) \geq \frac{1}{2}\left(d^{-}-1\right) d^{-}$! for non-melonic graphs, the following bound is true:

$$
\begin{aligned}
& \omega_{\mathrm{d} ; \times}\left(\mathcal{G}^{\text {melon }}\right) \leq-D\left(C_{\partial \mathcal{G}}-1\right)-\frac{1}{2}\left[\left(D d^{-}-2 b+4 a\right) N_{\mathrm{ext}}-2 D d^{-}\right] \\
& -2 b V_{2}-2 \sum_{\xi=a, 2 a}(b-\xi) V_{2 ; \xi}+\left(D d^{-}-4 b+4 a\right) V_{(4)}-2 a \triangle_{\times}^{\text {melon }}, \\
& \omega_{\mathrm{d} ; \times}\left(\mathcal{G}^{\text {non-melon }}\right) \leq-D\left(d^{-}-1\right)-D\left(C_{\partial \mathcal{G}}-1\right)-\frac{1}{2}\left[\left(D d^{-}-2 b+2 a\right) N_{\mathrm{ext}}-2 D d^{-}\right] \\
& -2 b V_{2}-2 \sum_{\xi=a, 2 a}(b-\xi) V_{2 ; \xi}+\left(D d^{-}-4 b+4 a\right) V_{(4)}-2 a \triangle_{\times}^{\text {non-melon }},
\end{aligned}
$$

where we define

$$
\begin{array}{r}
\Delta_{\times}^{\text {melon }}=2 V_{(4)}-N_{\text {ext }}-\rho_{\times}\left(\mathcal{G}^{\text {melon }}\right) \geq 0, \\
\Delta_{\times}^{\text {non-melon }}=2 V_{(4)}-\frac{N_{\text {ext }}}{2}-\rho_{\times}\left(\mathcal{G}^{\text {non-melon }}\right) \geq 0,
\end{array}
$$


and get the inequalities from Lemma 4. Thus, we obtain

$$
\begin{aligned}
& \omega_{\mathrm{d} ; \times}\left(\mathcal{G}^{\text {melon }}\right) \leq-D\left(C_{\partial \mathcal{G}}-1\right)-\frac{1}{2}\left[\left(D d^{-}-2 b+4 a\right) N_{\mathrm{ext}}-2 D d^{-}\right] \\
& -2 b V_{2}-2 \sum_{\xi=a, 2 a}(b-\xi) V_{2 ; \xi}+\left(D d^{-}-4 b+4 a\right) V_{(4)}, \\
& \omega_{\mathrm{d} ; \times}\left(\mathcal{G}^{\text {non-melon }}\right) \leq-D\left(d^{-}-1\right)-D\left(C_{\partial \mathcal{G}}-1\right)-\frac{1}{2}\left[\left(D d^{-}-2 b+2 a\right) N_{\mathrm{ext}}-2 D d^{-}\right] \\
& -2 b V_{2}-2 \sum_{\xi=a, 2 a}(b-\xi) V_{2 ; \xi}+\left(D d^{-}-4 b+4 a\right) V_{(4)} .
\end{aligned}
$$

Seeking renormalizable models, we require

$$
D d^{-}-4 b+4 a \leq 0, \quad 2 a \leq b,
$$

where the second condition, more stringent than $3 a \leq 2 b$, will be kept. This gives for $a$,

$$
a \leq b-\frac{1}{4} D d^{-}, \quad a \leq \frac{b}{2} .
$$

As in the previous case, let us see if we can achieve a just-renormalizable model with the condition $a=b-\frac{1}{4} D d^{-}$(which implies $b \leq \frac{D d^{-}}{2}$ ), (80), in (77) and (78) and require that, for a number of external legs higher than 4 , we have convergence

$$
\begin{gathered}
\left.\omega_{\mathrm{d} ; \times}\left(\mathcal{G}^{\text {melon }}\right)\right|_{N_{\text {ext }} \geq 6}<0, \\
\left.\omega_{\mathrm{d} ; \times}\left(\mathcal{G}^{\text {non-melon }}\right)\right|_{N_{\mathrm{ext}} \geq 6}<0 .
\end{gathered}
$$

From (77) and (78), we have

$$
\begin{aligned}
& \left.\omega_{\mathrm{d} ; \times}\left(\mathcal{G}^{\text {melon }}\right)\right|_{N_{\mathrm{ext}} \geq 6} \leq \\
& {\left.\left[-D\left(C_{\partial \mathcal{G}}-1\right)+D d^{-}-b N_{\mathrm{ext}}-2 b V_{2}-\frac{1}{2} D d^{-} V_{2 ; a}-\left(D d^{-}-2 b\right) V_{2 ; 2 a}\right]\right|_{N_{\mathrm{ext}} \geq 6}} \\
& \left.\omega_{\mathrm{d} ; \times}\left(\mathcal{G}^{\mathrm{non}-\mathrm{melon}}\right)\right|_{N_{\mathrm{ext}} \geq 6} \leq \\
& {\left.\left[D-D\left(C_{\partial \mathcal{G}}-1\right)-\frac{1}{4} D d^{-} N_{\mathrm{ext}}-2 b V_{2}-\frac{1}{2} D d^{-} V_{2 ; a}-\left(D d^{-}-2 b\right) V_{2 ; 2 a}\right]\right|_{N_{\mathrm{ext}} \geq 6}}
\end{aligned}
$$

The maximum value for $\omega_{\mathrm{d} ; \times}(\mathcal{G})$ is reached at $N_{\mathrm{ext}}=6$, so we can always write an upper bound and further require convergence

$$
\begin{aligned}
& \left.\omega_{\mathrm{d} ; \times}\left(\mathcal{G}^{\text {melon }}\right)\right|_{N_{\mathrm{ext}}=6} \leq D d^{-}-6 b<0, \\
& \left.\omega_{\mathrm{d} ; \times}\left(\mathcal{G}^{\text {non-melon }}\right)\right|_{N_{\mathrm{ext}}=6} \leq-D\left(\frac{3}{2} d^{-}-1\right)<0 .
\end{aligned}
$$

We note here that $d^{-}>\frac{2}{3}$ (85) is trivially satisfied in our study in which we only consider tensors with rank $d \geq 3$. Hence, still seeking just renormalizability, we impose

$$
\frac{D d^{-}}{6}<b \leq \frac{D d^{-}}{2}, \quad a=b-\frac{1}{4} D d^{-} .
$$

However (86) also entails $a>-\frac{D d^{-}}{12}$. Restricting to $a>0$, the bound of $b$ given can be improved. For just-renormalizability [i.e., the equality in (80), and (81) together with $a>0$ ], we impose

$$
\frac{D d^{-}}{4}<b \leq \frac{D d^{-}}{2}, \quad a=b-\frac{1}{4} D d^{-}>0,
$$

whose values for given positive integer values of $D$ and $d$ are given in Table III.

Let us understand what the condition $D d^{-}-4 b+4 a=0$ entails at $N_{\text {ext }}=4$. We have 
TABLE III. Allowed region of the values of $a$ and $b$ for potentially just-renormalizable models $\times$ with $d^{-} \leq 5$ and $D \leq 4$.

\begin{tabular}{|c|c|c|c|c|}
\hline & $d^{-}=2$ & $d^{-}=3$ & $d^{-}=4$ & $d^{-}=5$ \\
\hline \multirow{2}{*}{$D=1$} & $0<a \leq \frac{1}{2}$ & $0<a \leq \frac{3}{4}$ & $0<a \leq 1$ & $0<a \leq \frac{5}{4}$ \\
\hline & $\frac{1}{2}<b \leq 1$ & $\frac{3}{4}<b \leq \frac{3}{2}$ & $1<b \leq 2$ & $\frac{5}{4}<b \leq \frac{5}{2}$ \\
\hline \multirow{2}{*}{$D=2$} & $0<a \leq 1$ & $0<a \leq \frac{3}{2}$ & $0<a \leq 2$ & $0<a \leq \frac{5}{2}$ \\
\hline & $1<b \leq 2$ & $\frac{3}{2}<b \leq 3$ & $2<b \leq 4$ & $\frac{5}{2}<b \leq 5$ \\
\hline \multirow{2}{*}{$D=3$} & $0<a \leq \frac{3}{2}$ & $0<a \leq \frac{9}{4}$ & $0<a \leq 3$ & $0<a \leq \frac{15}{4}$ \\
\hline & $\frac{3}{2}<b \leq 3$ & $\frac{9}{4}<b \leq \frac{9}{2}$ & $3<b \leq 6$ & $\frac{15}{4}<b \leq \frac{15}{2}$ \\
\hline \multirow{2}{*}{$D=4$} & $0<a \leq 2$ & $0<a \leq 3$ & $0<a \leq 4$ & $0<a \leq 5$ \\
\hline & $2<b \leq 4$ & $3<b \leq 6$ & $4<b \leq 8$ & $5<b \leq 10$ \\
\hline
\end{tabular}

$$
\begin{aligned}
\left.\omega_{\mathrm{d} ; \times}\left(\mathcal{G}^{\text {non-melon }}\right)\right|_{N_{\mathrm{ext}}=4} \leq & -D\left(d^{-}-1\right)-D\left(C_{\partial \mathcal{G}}-1\right)-\frac{1}{2}\left[\frac{D d^{-}}{2} \cdot 4-2 D d^{-}\right] \\
& -2 b V_{2}-\frac{1}{2} D d^{-} V_{2 ; a}-\left(D d^{-}-2 b\right) V_{2 ; 2 a}, \\
\leq & -D\left(d^{-}-1\right)<0
\end{aligned}
$$

since we only consider tensors of rank $d \geq 3$. Thus, non-melonic graphs with $N_{\text {ext }}=4$ are found all convergent. Similarly for melonic graphs, requiring $D d^{-}-4 b+4 a=0$, leading to

$$
\begin{aligned}
& \left.\omega_{\mathrm{d} ; \times}\left(\mathcal{G}^{\text {melon }}\right)\right|_{N_{\mathrm{ext}}=4} \leq-D\left(C_{\partial \mathcal{G}}-1\right)-\frac{1}{2}\left[\left(2 a+\frac{D d^{-}}{2}\right) 4-2 D d^{-}\right] \\
& -2 b V_{2}-\frac{1}{2} D d^{-} V_{2 ; a}-\left(D d^{-}-2 b\right) V_{2 ; 2 a} \leq-4 a<0 .
\end{aligned}
$$

Therefore, all melonic graphs with $N_{\text {ext }}=4$ are also convergent. Therefore even though we have achieved $c_{(4)}=0$, the coefficient of $V_{(4)}$, (however in an inequality equation in the form of $\omega_{\mathrm{d}}(\mathcal{G}) \leq \cdots+\sum_{k} c_{k} V_{k}$ rather than with an equality), we cannot simply call $\phi^{4}$ (of any type, enhanced or not) coupling marginal in the standard sense. Furthermore, this model does not fall into the standard category of just-renormalizable theory because in the usual sense, it accompanies log-divergent amplitudes for $k_{\max }\left(=4\right.$ in this model) graphs. Indeed, if we insist to require that $N_{\text {ext }}=4$-point amplitudes could be log-divergent, we cannot achieve the $c_{(4)}=0$ condition (in the inequality equation for the divergence degree $\omega_{\mathrm{d}}(\mathcal{G})$ ). As a result, the upper bound on the divergence degree in (77) and (78) should be further worked out to determine the scaling dimensions of the $\phi^{4}$ couplings. However, this condition is strong enough to allow us to renormalize the theory. We therefore keep up the analysis.

Furthermore, we analyze graphs of $N_{\text {ext }}=2$ under the same condition and find

$$
\begin{aligned}
& \left.\omega_{\mathrm{d} ; \times \times}\left(\mathcal{G}^{\text {melon }}\right)\right|_{N_{\mathrm{ext}}=2} \leq-D\left(C_{\partial \mathcal{G}}-1\right)-\frac{1}{2}\left[2 b \cdot 2-2 D d^{-}\right] \\
& -2 b V_{2}-\frac{1}{2} D d^{-} V_{2 ; a}-\left(D d^{-}-2 b\right) V_{2 ; 2 a} \leq-2 b+D d^{-}<\frac{D d^{-}}{2} \\
& \left.\omega_{\mathrm{d} ; \times \times}\left(\mathcal{G}^{\text {non-melon }}\right)\right|_{N_{\mathrm{ext}}=2} \leq-D\left(d^{-}-1\right)-D\left(C_{\partial \mathcal{G}}-1\right)-\frac{1}{2}\left[\frac{D d^{-}}{2} \cdot 2-2 D d^{-}\right] \\
& -2 b V_{2}-\frac{1}{2} D d^{-} V_{2 ; a}-\left(D d^{-}-2 b\right) V_{2 ; 2 a} \leq \frac{1}{2} D\left(2-d^{-}\right) \leq 0,
\end{aligned}
$$

where we used (87) and $d^{-} \geq 2$. In summary, at $N_{\text {ext }}=2$, both melonic and non-melonic graphs might be divergent. A closer look shows that non-melonic graphs can be at most logarithmically divergent at rank $d \leq 3$. Furthermore, as observe above, if we increase $D$ or $d^{-}$, we see that melons could be again the dominant amplitudes. 
We conclude that, for potentially renormalizable models $\times$, i.e., under (80) and (81), only graphs with $N_{\text {ext }}=2$ might be divergent. Let us emphasize that the model $\times$ appears as a new type of renormalizable theory. Indeed, the coupling constants $\lambda$ and $\rho_{+}$do not get any corrections, i.e., do not get renormalized, but degree-2 vertices will do.

In ordinary QFT and invariant tensor field theory, when a model acquires this property it becomes super-renormalizable; that is, there is a finite number of graphs which contribute to the flow of the mass. That is, for example, the case of the scalar $P(\phi)_{2}$-model and the non-local super renormalizable tensor field theories. ${ }^{44,47}$ However, in the present case, as we will see in the following, the model $X$ at $d=3$ will have an infinite number of graphs which will contribute to the mass renormalization. We attribute this property to the presence of enhanced interactions in the model. As a concrete study in Sec. VII, we will focus on $a=\frac{1}{2}, b=1$ for $D=1$ and $d^{-}=2$ as satisfied in Table III.

\section{RANK $\boldsymbol{d}$ JUST-RENORMALIZABLE MODELS +}

In this section, we analyze a class of model + which will be proved renormalizable for arbitrary $d$ and $D$. We provide the list of their primitively divergent graphs and proceed to the expansion of those around their local and diverging part. Our goal is to show that the divergent parts in this expansion recast as a coupling and so a subtracting scheme can be performed. Dealing exclusively with graphs with external legs, we have $C_{\partial \mathcal{G}} \geq 1$. Note also that the theory has bipartite graphs such that $N_{\text {ext }}$ is an even number.

\section{A. List of divergent graphs}

Consider an arbitrary model in the class of models + at fix $(d, D)$, with $a=D\left(d^{-}-1\right) / 2, b$ $=D\left(d^{-}-\frac{1}{2}\right) / 2$. Using (55) and (57), in the same notations and conditions introduced above, the superficial degree of divergence is given by

$$
\begin{gathered}
\omega_{\mathrm{d} ;+}\left(\mathcal{G}^{\text {melon }}\right) \leq-D\left[\left(C_{\partial \mathcal{G}}-1\right)+\frac{1}{2}\left(\left(d^{-}-\frac{1}{2}\right) N_{\mathrm{ext}}-2 d^{-}\right)+\left(d^{-}-\frac{1}{2}\right) V_{2}+\frac{1}{2} V_{2 ; a}+\left(d^{-}-1\right) \Delta_{+}^{\text {melon }}\right], \\
\omega_{\mathrm{d} ;++}\left(\mathcal{G}^{\text {non-melon }}\right) \leq-D\left[\left(d^{-}-1\right)+\left(C_{\partial \mathcal{G}}-1\right)+\frac{1}{2}\left(\frac{1}{2} N_{\mathrm{ext}}-2 d^{-}\right)\right. \\
\left.+\left(d^{-}-\frac{1}{2}\right) V_{2}+\frac{1}{2} V_{2 ; a}+\left(d^{-}-1\right) V_{4}+\left(d^{-}-1\right) \triangle_{+}\right] .
\end{gathered}
$$

We have already shown that for any graph such that $N_{\text {ext }} \geq 6$, the amplitude is convergent. At $N_{\text {ext }}=4$, non-melonic graphs have maximal degree of divergence 0 (logarithmic divergence) and melonic graphs converge.

The following cases occur

(i) $N_{\text {ext }}=4$,

$$
\begin{aligned}
& \omega_{\mathrm{d} ;+}\left(\mathcal{G}^{\text {melon }}\right) \leq-D\left(d^{-}-1\right)<0, \\
& \omega_{\mathrm{d} ;+}\left(\mathcal{G}^{\text {non-melon }}\right) \leq-D\left[\left(C_{\partial \mathcal{G}}-1\right)+\left(d^{-}-\frac{1}{2}\right) V_{2}+\frac{1}{2} V_{2 ; a}+\left(d^{-}-1\right) V_{4}\right. \\
& \left.+\left(d^{-}-1\right) \triangle_{+}\right] \leq 0 .
\end{aligned}
$$

For non-melonic graphs, the upper bound saturates only if $C_{\partial \mathcal{G}}=1, V_{4}=V_{2}=V_{2 ; a}=0$, and $\Delta_{+}=0$, i.e., $\rho_{+}\left(\mathcal{G}^{\text {non-melon }}\right)=V_{+; 4}$.

(ii) $\quad N_{\text {ext }}=2$ : we can combine $V_{(4)}>1$ and $V_{(4)}=1$ at $N_{\text {ext }}=2$ from Lemma 3. Thus we can write a single bound, $V_{(4)} \geq 1$ as

$$
\omega_{\mathrm{d} ;+}\left(\mathcal{G}^{\text {melon }}\right) \leq-D\left[\left(C_{\partial \mathcal{G}}-1\right)-\frac{1}{2}+\left(d^{-}-\frac{1}{2}\right) V_{2}+\frac{1}{2} V_{2 ; a}+\left(d^{-}-1\right) \triangle_{+}^{\text {melon }}\right] \leq \frac{D}{2} .
$$

Whenever $C_{\partial \mathcal{G}}-1>0$, or $V_{2}>0, V_{2 ; a}>1$, or $\Delta_{+}^{\text {melon }}>0$, the graph becomes convergent. The only way to achieve a divergence with $\omega_{\mathrm{d} ;+}\left(\mathcal{G}^{\text {melon }}\right)=\frac{D}{2}$ is to set the above quantities 
to 0 . Note that for a graph with $N_{\text {ext }}=2, \Delta_{+}^{\text {melon }}=0$ means $\rho_{+}\left(\mathcal{G}^{\text {melon }}\right)=V_{(4)}-1$ from Lemma 3. But we also have the bound $\rho_{+}\left(\mathcal{G}^{\text {melon }}\right) \leq V_{+; 4}$; therefore, writing $\rho_{+}\left(\mathcal{G}^{\text {melon }}\right)=V_{+; 4}-p$, $p \geq 0$, implies that $V_{4}=1-p \geq 0$. Thus $p=0$ yields $\left(\rho_{+}\left(\mathcal{G}^{\text {melon }}\right)=V_{+; 4}, V_{4}=1\right)$ or $p=1$ and then $\left(\rho_{+}\left(\mathcal{G}^{\text {melon }}\right)=V_{+; 4}-1, V_{4}=0\right)$.

The case $\omega_{\mathrm{d} ;+}\left(\mathcal{G}^{\text {melon }}\right)=0$ might occur for $C_{\partial \mathcal{G}}-1=0, V_{2}=0, \Delta_{+}^{\text {melon }}=0$, and $V_{2 ; a}=1$. Then $\Delta_{+}^{\text {melon }}=0$ means that one of the following two cases occurs, $\left(\rho_{+}\left(\mathcal{G}^{\text {melon }}\right)=V_{+; 4}, V_{4}=1\right)$ or $\left(\rho_{+}\left(\mathcal{G}^{\text {melon }}\right)=V_{+; 4}-1, V_{4}=0\right)$ can be produced.

For a non-melonic graph, we have $V_{(4)} \geq 0$,

$$
\begin{aligned}
& \omega_{\mathrm{d} ;+}\left(\mathcal{G}^{\text {non-melon }}\right) \leq-D\left[\left(C_{\partial \mathcal{G}}-1\right)-\frac{1}{2}\right. \\
& \left.+\left(d^{-}-\frac{1}{2}\right) V_{2}+\frac{1}{2} V_{2 ; a}+\left(d^{-}-1\right) V_{4}+\left(d^{-}-1\right) \Delta_{+}\right] \leq \frac{D}{2},
\end{aligned}
$$

and the only way to achieve divergence is to set $C_{\partial \mathcal{G}}=1, V_{2}=0, V_{4}=0$, and $\triangle_{+}=0$. The last condition translates as $\rho_{+}\left(\mathcal{G}^{\text {melon }}\right)=V_{+; 4}$. Likewise, we can have $\omega_{\mathrm{d} ;+}\left(\mathcal{G}^{\text {non-melon }}\right)=\frac{D}{2}$ for $V_{2 ; a}=0$, or $\omega_{\mathrm{d} ;+}\left(\mathcal{G}^{\text {non-melon }}\right)=0$ for $V_{2 ; a}=1$.

We have thus completed the proof of the following statement:

Proposition 1 (List of primitively divergent graphs for model + ). The $p^{2 a} \phi^{4}$-model + with parameters $a=D\left(d^{-}-1\right) / 2, b=D\left(d^{-}-\frac{1}{2}\right) / 2$ for two integers $d>2$ and $D>0$ has primitively divergent graphs with $\left(\Omega(\mathcal{G})=\omega\left(\mathcal{G}_{\text {color }}\right)-\omega(\partial \mathcal{G})\right)$. See Table IV.

Some divergent 2-point graphs are illustrated in Figs. 6 and 7 in Appendix B specializing to $d=3$ and $D=1$. They will contribute to the mass renormalization for this model. Second, consider the 4-point amplitudes associated with the graphs of Fig. 8 in Appendix B. These will contribute to the renormalization of couplings $\eta_{+}$or $\lambda$ depending on the external momentum data of the correlators. We can construct an infinite family of divergent 4-point graphs in this model.

At the end of this section, the proof of the next theorem will be completed.

Theorem 1. The $p^{2 a} \phi^{4}$ model + with parameters $a=D\left(d^{-}-1\right) / 2, b=D\left(d^{-}-\frac{1}{2}\right) / 2$ for arbitrary rank $d \geq 3$ and dimension $D>0$ with action defined by (2) is just-renormalizable at all orders of perturbation theory.

\section{B. Renormalization}

The subsequent part of the renormalization program consists in the proof that the divergent and local part of all divergent amplitudes can be recast as terms which are present in the Lagrangian of the model + of Sec. VI A with fixed parameter $a=D\left(d^{-}-1\right) / 2$ and $b=D\left(d^{-}-1 / 2\right) / 2$. For that purpose, we perform a Taylor expansion of the amplitudes of graphs listed in Table IV and show that the divergent terms in that expansion are associated with either the mass, counter-terms $C T_{2 ; \xi}$, $\xi=a, b$, or interaction terms plus convergent remainders.

TABLE IV. List of primitively divergent graphs of the $p^{2 a} \phi^{4}$-model + .

\begin{tabular}{lcccccccc}
\hline \hline $\mathcal{G}$ & $N_{\text {ext }}$ & $V_{2}$ & $V_{2 ; a}$ & $V_{4}$ & $\rho_{+}$ & $C_{\partial \mathcal{G}}-1$ & $\Omega(\mathcal{G})$ & $\omega_{\mathrm{d} ;+}(\mathcal{G})$ \\
\hline & 4 & 0 & 0 & 0 & $V_{+; 4}$ & 0 & 1 & 0 \\
I & 2 & 0 & 0 & 0 & $V_{+; 4}$ & 0 & 1 & $\frac{D}{2}$ \\
II & 2 & 0 & 0 & 0 & $V_{+; 4}-1$ & 0 & 0 & $\frac{D}{2}$ \\
III & 2 & 0 & 0 & 1 & $V_{+; 4}$ & 0 & 0 & $\frac{D}{2}$ \\
IV & 2 & 0 & 1 & 0 & $V_{+; 4}$ & 0 & 1 & 0 \\
V & 2 & 0 & 1 & 0 & $V_{+; 4}-1$ & 0 & 0 & 0 \\
VI & 2 & 0 & 1 & 1 & $V_{+; 4}$ & 0 & 0 & 0 \\
\hline \hline
\end{tabular}




\section{Renormalization of marginal 4-point functions}

Marginal 4-point functions are given by the first line of Table IV. Given a connected and bipartite boundary graph of a 4-point graph, it is simple to realize that the pattern of its external momenta should follow either the pattern of $\mathbf{V}_{4 ; s}$ or of $\mathbf{V}_{+; 4 ; s}$ (15) (see Fig. 2). The locality principle of the present model tells us to consider a graph issued from the expansion of correlators of the form (17) or (18) which translates as

$$
\begin{aligned}
& \left\langle\phi_{12 \ldots d} \bar{\phi}_{1^{\prime} 2 \ldots d} \phi_{1^{\prime} 2^{\prime} \ldots d^{\prime}} \bar{\phi}_{12^{\prime} \ldots d^{\prime}}\right\rangle, \\
& \left\langle\left|p_{1}^{\mathrm{ext}}\right|^{2 a} \bar{\phi}_{1^{\prime} 2 \ldots d} \phi_{1^{\prime} 2^{\prime} \ldots d^{\prime}} \bar{\phi}_{12^{\prime} \ldots d^{\prime}}\right\rangle,
\end{aligned}
$$

with $\left|p_{1}^{\text {ext }}\right|^{2 a}$ an external momentum with color $s=1$. In the following, we will concentrate on an expansion of a graph with external data of the form of the operator $\mathbf{V}_{+; 4 ; s=1}$. In other words, we will focus on $s=1$ and a graph coming from the expansion of the correlator (98). However, as it will be clear, our analysis is without loss of generality since the method can be extended to $\mathbf{V}_{4 ; 1}$ and then to $\mathbf{V}_{+; 4 ; s}$, for any color $s$.

Consider a 4-point graph with 4 external propagators attached to it with external momenta governed by the pattern of (98). This 4-point graph carries $2 d$ momentum labels; these are associated with $2 d$ external faces, which we denote by

$$
f \in \mathcal{F}_{\text {ext }}=\left\{f_{[1]}, f_{2}, \ldots, f_{d}, f_{1^{\prime}}, f_{2^{\prime}}, \ldots, f_{d^{\prime}}\right\},
$$

where we emphasize the face which is enhanced by a square bracket (say, [1]). Let $A_{4}\left(\left\{p_{f}^{\mathrm{ext}}\right\}\right)$ be the amplitude of such a graph. Two types of scale indices have to be considered in this amplitude: the external scales $j_{l}$ associated with external fields and which correspond to external propagators with labels $l$ and the (internal) scale $i$ of the $G_{k}^{i}$ graph. In short, a quasi-local graph $G_{k}^{i}$ implies that $j_{l} \ll i$.

We have from (25)

$$
\begin{aligned}
& A_{4}\left(\left\{p_{f_{s}}^{\mathrm{ext}}\right\}\right)=\kappa\left(\eta_{+}\right) \sum_{p_{f_{s}}} \int\left[\prod_{l \in \mathcal{L}} d \alpha_{l} e^{-\alpha_{l} \mu}\right]\left[\prod_{f_{s} \in \mathcal{F}_{\mathrm{ext}}} e^{-\left(\sum_{l \in f_{s}} \alpha_{l}\right) \sum_{\xi}\left|p_{f_{s}}^{\mathrm{ext}}\right|^{2 \xi}}\right] \\
& \times\left[\prod_{f_{s} \in \mathcal{F}_{\text {int }}} e^{-\left(\sum_{l \in f_{s}} \boldsymbol{\alpha}_{l}\right) \sum \xi\left|p_{f_{s}}\right|^{2 \xi}}\right]\left|p_{f_{[1]}}^{\mathrm{ext}}\right|^{2 a}\left[\prod_{s=1}^{d} \prod_{v_{s} \in \mathcal{V}_{+; 4 ; s}}\left(\epsilon \tilde{p}^{2 a}\right)_{v_{s}}\right] \text {, } \\
& \left(\epsilon \tilde{p}^{2 a}\right)_{v_{s}}:=\sum_{f_{s^{\prime}}} \epsilon_{v_{s} \cdot f_{s^{\prime}}}\left(\tilde{p}_{f_{s^{\prime}}}\right)^{2 a} \text {, }
\end{aligned}
$$

where $\kappa\left(\eta_{+}\right)$includes symmetry factors and coupling constants. We recall that $p_{f_{s}}^{\text {ext }}$ are external momenta, and the last line shows $\tilde{p}_{f_{s}}$ which refers to an internal or an external momentum. Let us concentrate on the range of the parameters $\alpha$ : for an internal line $l$, that we will now denote $\ell$, $\alpha_{\ell} \in\left[M^{-(2 b) i_{\ell}}, M^{-(2 b)\left(i_{\ell}-1\right)}\right]$; for an external line $l$, now denoted $l_{\mathrm{ext}}, \alpha_{l_{\mathrm{ext}}} \in\left[M^{-(2 b) j_{\mathrm{lext}}}, M^{-(2 b)\left(j_{\mathrm{ext}}-1\right)}\right]$. We are interested in a regime when $j_{l_{\text {ext }}} \ll i \leq i_{\ell}$.

A Taylor expansion over an external face amplitude gives

$$
\begin{aligned}
e^{-\left(\sum_{l \in f} \alpha_{l}\right) \sum \xi\left|p_{f}^{\mathrm{ext}}\right|^{2 \xi}} & =e^{-\left(\alpha_{l \mathrm{ext}}+\alpha_{l \mathrm{ext}}{ }^{\prime}\right) \sum \xi\left|p_{f}^{\mathrm{ext}}\right|^{2 \xi}}\left[1-R_{f}\right], \\
R_{f} & =\left(\sum_{\ell \in f} \alpha_{\ell}\right)\left(\sum_{\xi}\left|p_{f}^{\mathrm{ext}}\right|^{2 \xi}\right) \int_{0}^{1} e^{-t\left(\sum_{\ell \in f} \alpha_{\ell}\right) \sum \xi\left|p_{f}^{\mathrm{ext}}\right|^{2 \xi}} d t,
\end{aligned}
$$

where $\sum_{\ell \in f} \alpha_{\ell}$ is small $\left(\alpha_{\ell} \sim \mathcal{O}\left(\frac{1}{\left|p_{f_{s}}\right|^{2 \xi}}\right) \sim M^{-(2 \xi) i_{\ell}}\right)$. We insert that expansion for each external face in (99) and obtain

$$
\begin{aligned}
& A_{4}\left(\left\{p_{f}^{\mathrm{ext}}\right\}\right)=\kappa\left(\eta_{+}\right) \sum_{p_{f}} \int\left[\prod_{l \in \mathcal{L}} d \alpha_{l} e^{-\alpha_{l} \mu}\right]\left|p_{f_{[1]}}^{\mathrm{ext}}\right|^{2 a}\left[\prod_{f \in \mathcal{F}_{\mathrm{ext}}} e^{-\left(\alpha_{l \mathrm{ext}}+\alpha_{l \mathrm{ext}}{ }^{\prime}\right) \sum_{\xi}\left|p_{f}^{\mathrm{ext}}\right|^{2 \xi}}\right] \\
& \times\left[1-\sum_{f \in \mathcal{F}_{\text {ext }}} R_{f}+\sum_{f, f^{\prime} \in \mathcal{F}_{\text {ext }}} R_{f} R_{f^{\prime}}+\ldots\right]\left[\prod_{f \in \mathcal{F}_{\text {int }}} e^{-\left(\sum_{\ell \in f} \alpha_{\ell}\right) \sum_{\xi}\left|p_{f}\right|^{2 \xi}}\right] \prod_{s=1}^{d} \prod_{v_{s} \in \mathcal{V}_{+; ; ; s}}\left[\left(\epsilon \tilde{p}^{2 a}\right)_{v_{s}}\right] .
\end{aligned}
$$


The dots are higher order products in the $R_{f}$ 's. From Table IV, $\rho_{+}(34)$ must be equal to $V_{+; 4}$. Hence, in each vertex kernel, we must collect and integrate one momentum of a closed face. A divergent 4-point graph satisfying the first row of Table IV must be such that no external momenta can be found within $\prod_{s=1}^{d} \prod_{v_{s} \in \mathcal{V}_{+; 4 ; s}}\left[\left(\epsilon \tilde{p}^{2 a}\right)_{v_{s}}\right]$.

We write the 0th order in expansion in $R_{f}$ as

$$
\begin{aligned}
& A_{4}\left(\left\{p_{f}^{\mathrm{ext}}\right\} ; 0\right)=\kappa\left(\eta_{+}\right) \sum_{p_{f}} \int\left[\prod_{l} d \alpha_{l} e^{-\alpha_{l} \mu}\right]\left|p_{f_{[1]}}^{\mathrm{ext}}\right|^{2 a} \prod_{f \in \mathcal{F}_{\mathrm{ext}}}\left[\left.e^{-\left(\alpha_{\text {ext }}+\alpha_{l \mathrm{ext}}\right) \sum \xi \mid p_{f}^{\mathrm{ext}}}\right|^{2 \xi}\right] \\
& \times\left[\prod_{f \in \mathcal{F}_{\text {int }}} e^{-\left(\sum_{\ell \in f} \alpha_{\ell}\right) \sum_{\xi}\left|p_{f}\right|^{2 \xi}}\right]\left[\prod_{s=1}^{d} \prod_{v_{s} \in \mathcal{V}_{+; ; ; s}}\left(\epsilon \tilde{p}^{2 a}\right)_{v_{s}}\right], \\
& =\kappa\left(\eta_{+}\right)\left[\int\left[\prod_{l_{\mathrm{ext}}} d \alpha_{l_{\mathrm{ext}}} e^{-\alpha_{l_{\mathrm{ext}}} \mu}\right]\left|p_{f_{[1]}^{\mathrm{ext}}}\right|^{2 a} \prod_{f \in \mathcal{F}_{\mathrm{ext}}} e^{-\left(\alpha_{l_{\mathrm{ext}}}+\alpha_{l_{\mathrm{ext}}}\right)^{\prime} \sum_{\xi}\left|p_{f}^{\mathrm{ext}}\right|^{2 \xi}}\right] \\
& \times\left[\sum_{p_{f}} \int\left[\prod_{\ell} d \alpha_{\ell} e^{-\alpha_{\ell} \mu}\right] \prod_{f \in \mathcal{F}_{\text {int }}}\left[e^{-\left(\sum_{\ell} \alpha_{\ell}\right) \sum_{\xi}\left|p_{f}\right|^{2 \xi}}\right]\left[\prod_{s=1}^{d} \prod_{v_{s} \in \mathcal{V}_{+; ; ; s}}\left(\epsilon \tilde{p}^{2 a}\right)_{v_{s}}\right]\right] .
\end{aligned}
$$

The factor involving external lines can be re-expressed as

$$
\begin{aligned}
& \int\left[\prod_{l_{\mathrm{ext}}} d \alpha_{l_{\mathrm{ext}}} e^{-\alpha_{l_{\mathrm{ext}}} \mu}\right]\left|p_{f_{[1]}}^{\mathrm{ext}}\right|^{2 a} \prod_{f \in \mathcal{F}_{\mathrm{ext}}} e^{-\left(\alpha_{\text {lext }}+\alpha_{\text {lext }}{ }^{\prime}\right) \sum \xi\left|p_{f}^{\mathrm{ext}}\right|^{2 \xi}}= \\
& \int\left[\prod_{l_{\mathrm{ext}}} d \alpha_{l_{\mathrm{ext}}}\right]\left|p_{f_{[1]}}^{\mathrm{ext}}\right|^{2 a} \\
& \times e^{-\alpha_{l_{\text {ext } 1}}\left[\sum_{\xi}\left(\left|p_{f_{[1]}}^{\text {ext }}\right|^{2 \xi}+\left|p_{f_{2}}^{\text {ext }}\right|^{2 \xi}+\cdots+\left|p_{f_{d}}^{\text {ext }}\right|^{2 \xi}\right)+\mu\right]} e^{-\alpha_{l_{\text {ext } 2}}\left[\sum_{\xi}\left(\left|p_{f_{1}}^{\text {ext }}\right|^{2 \xi}+\left|p_{f_{2}}^{\text {ext }}\right|^{2 \xi}+\cdots+\left|p_{f_{d}}^{\text {ext }}\right|^{2 \xi}\right)+\mu\right]}
\end{aligned}
$$

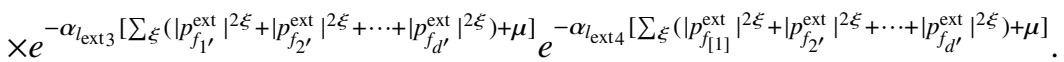

Observe that the above expression describes 4 propagators glued in a way to produce the pattern of a vertex of type $\mathbf{V}_{+; 4 ; 1}$. The term associated with the sum over internal momenta is log-divergent. Therefore, the amplitude $A_{4}\left(\left\{p_{f}^{\mathrm{ext}}\right\} ; 0\right)$ will renormalize the coupling $\eta_{+}$.

We now prove that the remainders appearing in (101) lead to convergence by improving the power counting. The first remainder calling a single term $R_{f}$ is of the form

$$
\begin{aligned}
& R_{4}=\kappa\left(\eta_{+}\right) \sum_{p_{f}} \int\left[\prod_{l} d \alpha_{l} e^{-\alpha_{l} \mu}\right]\left|p_{f_{[1]}}^{\text {ext }}\right|^{2 a}\left[\prod_{f \in \mathcal{F}_{\text {ext }}} e^{-\left(\alpha_{l \text { ext }}+\alpha_{l \text { ext }}{ }^{\prime}\right) \sum_{\xi}\left|p_{f}^{\text {ext }}\right|^{2 \xi}}\right] \\
& \times\left[-\sum_{f \in \mathcal{F}_{\mathrm{ext}}}\left(\sum_{\ell \in f} \alpha_{\ell}\right)\left(\sum_{\xi}\left|p_{f}^{\mathrm{ext}}\right|^{2 \xi}\right) \int_{0}^{1} e^{-t\left(\sum_{\ell \in f} \alpha_{\ell}\right) \sum_{\xi}\left|p_{f}^{\mathrm{ext}}\right|^{2 \xi}} d t\right]
\end{aligned}
$$

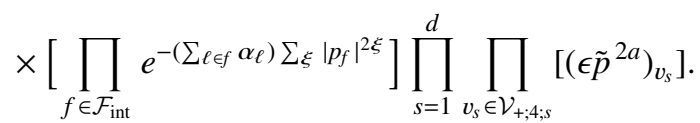

Using $i\left(G_{k}^{i}\right)=\inf _{\ell \in G_{k}^{i}} i_{\ell}$ and $e\left(G_{k}^{i}\right)=\sup _{l \in G_{k}^{i}} j_{l}$ and recalling $\alpha_{\ell} \in\left[M^{-(2 b) i_{\ell}}, M^{-(2 b)\left(i_{\ell}-1\right)}\right]$ and $\alpha_{l_{\mathrm{ext}}} \in$ $\left[M^{-(2 b) j_{l \mathrm{ext}}}, M^{-(2 b)\left(j_{l \mathrm{ext}}-1\right)}\right],\left(\sum_{\ell \in f} \alpha_{\ell}\right)\left|p_{f}^{\mathrm{ext}}\right|^{2 b} \leq k_{0} M^{-(2 b)\left(i\left(G_{k}^{i}\right)-e\left(G_{k}^{i}\right)\right)}$, then $R_{4}$ is bounded as

$$
\left|R_{4}\right| \leq K \prod_{(i, k)} M^{-(2 b)\left(i\left(G_{k}^{i}\right)-e\left(G_{k}^{i}\right)\right)} M^{\omega_{\mathrm{d} ;+}\left(G_{k}^{i}\right)},
$$

for some constant $K$ (which includes $\kappa\left(\eta_{+}\right)$and $k_{0}$, a constant depending on the graph and a constant which bounds the integral in $t$ ). The factor $M^{-(2 b)\left(i\left(G_{k}^{i}\right)-e\left(G_{k}^{i}\right)\right)}$ improves the power counting (which is already logarithmic) and will be the source of decay to show that the sum over scale attributions 
is convergent in the way established in Ref. 68. Inspecting the higher order products in $R_{f}$ 's, one realizes that they are obviously more convergent and so do not need further discussion.

If we remove $\left|p_{f_{[1]}}^{\text {ext }}\right|^{2 a}$ from the amplitude (99), we will be in the presence of an amplitude coming from (97). The boundary data of the resulting amplitude will be of the form $\mathbf{V}_{4 ; 1}$. Repeating step by step the previous analysis, we obtain at 0th order of the expansion a renormalization of the coupling $\lambda$ and all remainders will lead exactly to the same convergence with power counting given by (105). It is direct to see that the argument extends to any color $s$.

As a result of this analysis, we can comment that, although the renormalized coupling $\lambda$ does not receive any melonic corrections, it receives contributions from the coupling $\eta_{+}$. This is a new property of the perturbative Renormalization Group equations of this model.

\section{Renormalization of divergent 2-point functions}

We study 2-point functions that obey $\omega_{\mathrm{d} ;+}(\mathcal{G})=0, \frac{D}{2}$ and characterized by the rows I through VI of Table IV. First, we will focus on the row I and point out the differences with II and III at particular steps of the discussion. We will sketch the analysis for the rows IV, V, and VI.

A 2-point graph has a unique boundary which is given by the invariant $\operatorname{Tr}_{2}\left(\phi^{2}\right)$ of Fig. 2. Because the vertices are enhanced, it may happen that the external data of a two-point graph are not of the form of a mass term, but rather a form of $C T_{2 ; a}$. A careful discussion will be made about this in the text.

Consider an amplitude $A_{2}\left(\left\{p_{f}^{\text {ext }}\right\}\right)$ associated with a 2-point graph obeying the row I of Table IV. This graph has $d$ external faces that we label by

$$
f \in \mathcal{F}_{\text {ext }}=\left\{f_{1}, f_{2}, \ldots, f_{d}\right\} .
$$

Keeping the same notations as above [see paragraph after (99)], $l_{\text {ext }}$ labels external propagators with the scale index $j_{l_{\text {ext }}}, \ell$ labels internal lines with the scale index $i_{\ell}$.

A Taylor expansion of the external face factors out in the same form as (100) and leads us to the following expansion of the 2-point amplitude:

$$
\begin{aligned}
& A_{2}\left(\left\{p_{f}^{\mathrm{ext}}\right\}\right)=\kappa\left(\eta_{+}\right) \sum_{p_{f}} \int\left[\prod{ }_{l} d \alpha_{l} e^{-\alpha_{l} \mu}\right]\left[\prod_{f \in \mathcal{F}_{\text {ext }}} e^{\left.-\left(\alpha_{l \mathrm{ext}}+\alpha_{l \mathrm{ext}}\right) \sum_{\xi}\left|p_{f}^{\mathrm{ext}}\right|^{2 \xi}\right]}\right. \\
& \times\left[1-\sum_{f \in \mathcal{F}_{\text {ext }}} R_{f}+\sum_{f, f^{\prime} \in \mathcal{F}_{\text {ext }}} R_{f} R_{f^{\prime}}+\ldots\right]\left[\prod_{f \in \mathcal{F}_{\text {int }}} e^{-\left(\sum_{\ell \in f} \alpha_{\ell}\right) \sum_{\xi}\left|p_{f}\right|^{2 \xi}}\right]\left[\prod_{s=1}^{d} \prod_{v_{s} \in \mathcal{V}_{+; 4 ; s}}\left[\left(\epsilon \tilde{p}^{2 a}\right)_{v_{s}}\right] .\right.
\end{aligned}
$$

The 0th order term in the expansion in $R_{f}$

$$
\begin{aligned}
& A_{2}\left(\left\{p_{f}^{\mathrm{ext}}\right\} ; 0\right)=\kappa\left(\eta_{+}\right) \sum_{p_{f}} \int\left[\prod_{l} d \alpha_{l} e^{-\alpha_{l} \mu}\right] \\
& \times\left[\prod_{f \in \mathcal{F}_{\text {ext }}} e^{-\left(\alpha_{\text {lext }}+\alpha_{l_{\text {ext }}}\right) \sum_{\xi}\left|p_{f}^{\text {ext }}\right|^{2 \xi}}\right] \prod_{f \in \mathcal{F}_{\text {int }}}\left[e^{\left.-\left(\sum_{\ell \in f} \alpha_{\ell}\right) \sum_{\xi}\left|p_{f}\right|^{2 \xi}\right]} \prod_{s=1}^{d} \prod_{v_{s} \in \mathcal{V}_{+; 4 ; s}}\left[\left(\epsilon \tilde{p}^{2 a}\right)_{v_{s}}\right]\right. \\
& =\kappa\left(\eta_{+}\right)\left[\int\left[\prod_{l_{\mathrm{ext}}} d \alpha_{l_{\mathrm{ext}}} e^{-\alpha_{l \mathrm{ext} 1}\left[\sum_{\xi}\left(\sum_{s}\left|p_{f_{s}}^{\mathrm{ext}}\right|^{2 \xi}\right)+\mu\right]} e^{-\alpha_{l_{\mathrm{ext} 2}}\left[\sum_{\xi}\left(\sum_{s}\left|p_{f_{s}}^{\mathrm{ext}}\right|^{2 \xi}\right)+\mu\right]}\right]\right. \\
& \times\left[\sum_{p_{f}} \int\left[\prod_{\ell} d \alpha_{\ell} e^{-\alpha_{\ell} \mu}\right] \prod_{f \in \mathcal{F}_{\text {int }}}\left[e^{-\left(\sum_{\ell} \alpha_{\ell}\right) \sum_{\xi}\left|p_{f}\right|^{2 \xi}}\right]\left[\prod_{s=1}^{d} \prod_{v_{s} \in \mathcal{V}_{+; ; ; s}}\left(\epsilon \tilde{p}^{2 a}\right)_{v_{s}}\right]\right] .
\end{aligned}
$$

Let us discuss the vertex density. A graph fulfilling the requirements of the row I of Table IV, must be such that $\rho_{+}=V_{+; 4}$. The same argument as in the case of 4-point functions applies: there are no external momenta in the product of vertex kernels and all momenta will be summed. The first factor involving external momenta represents two propagators glued together by a degree- 2 vertex; 
the second factor gives by our power counting a degree of divergence $\frac{D}{2}$. Hence the term (107) renormalizes the mass term.

Concerning a graph satisfying the rows II and III, we know that $\rho_{+}=V_{(4)}-1$ and that the graph is melonic. Lemma 3 explained in its proof that for 1PI melonic graphs, external legs must be hooked on partner vertices. Hence a 2-point primitively divergent (1PI) melonic graph has at least a vertex $v_{0 ; s}$ which will not contribute to $\rho_{+}$. Two cases might occur:

(II) The vertex $v_{0 ; s}$ must belong to $\mathcal{V}_{+; 4 ; s}$, then an extra factor of $\left|p_{f_{s}}^{\text {ext }}\right|^{2 a}$ must be added to the boundary data. This makes that graph of the form of $\mathcal{V}_{2 ; a ; s}$. Now, adding all colored symmetric contribution with respect to $s$ of this graph term renormalizes the coupling $Z_{a}$;

(III) The vertex $v_{0 ; s}$ must belong to $\mathcal{V}_{4}$, then the boundary data is that of a mass and so (107) again renormalizes the mass term.

The remainders of (106) are now treated for the row I of our table. The first order remainder involving the sum $\sum_{f} R_{f}$ can be bounded as follows:

$$
\begin{aligned}
& R_{2}=-\kappa\left(\eta_{+}\right)\left[\int\left[\prod_{l_{\mathrm{ext}}} d \alpha_{l_{\mathrm{ext}}} e^{-\alpha_{l \mathrm{ext}} \mu}\right] \prod_{f \in \mathcal{F}_{\mathrm{ext}}} e^{-\left(\alpha_{l_{\mathrm{ext}}}+\alpha_{l_{\mathrm{ext}}{ }^{\prime}}\right) \sum_{\xi}\left|p_{f}^{\mathrm{ext}}\right|^{2 \xi}}\right] \\
& \times \sum_{f \in \mathcal{F}_{\text {ext }}}\left[\int\left[\prod_{\ell} d \alpha_{\ell} e^{-\alpha_{\ell} \mu}\right]\left(\sum_{\ell \in f} \alpha_{\ell}\right)\left(\sum_{\xi}\left|p_{f}^{\text {ext }}\right|^{2 \xi}\right) \int_{0}^{1} e^{-t\left(\sum_{\ell \in f} \alpha_{\ell}\right) \sum_{\xi}\left|p_{f}^{\text {ext }}\right|^{2 \xi}} d t\right] \\
& \times \sum_{p_{f}} \prod_{f \in \mathcal{F}_{\text {int }}}\left[e^{\left.-\left(\sum_{\ell \in f} \alpha_{\ell}\right) \Sigma_{\xi}\left|p_{f}\right|^{2 \xi}\right]}\right] \prod_{s=1}^{d} \prod_{v_{s} \in \mathcal{V}_{+; ; ; s}}\left[\left(\epsilon \tilde{p}^{2 a}\right)_{v_{s}}\right] \\
& \left|R_{2}\right| \leq K \prod_{(i, k)} M^{-2 b\left(i\left(G_{k}^{i}\right)-e\left(G_{k}^{i}\right)\right)} M^{\omega_{\mathrm{d} ;}\left(G_{k}^{i}\right)=\frac{D}{2}},
\end{aligned}
$$

where we used the same scheme leading to (105), for some constant $K$. For a constant $C \geq 1$, $-D\left(d^{-}-\frac{1}{2}\right) C+\frac{D}{2} \leq-D\left(d^{-}-1\right) \leq-D<0$ ensures the convergence of the remainder. All higher order remainders can be proved to be more convergent. From this point, the summation over scale attributions can be again performed after subtractions. The case of an amplitude of the rows II and III of the table can be addressed in a similar way.

Let us now discuss the rows IV, V, and VI. In these cases, $\rho_{2 ; a}=1=V_{2 ; a}$ which means that the enhanced momenta $\left|p_{f_{s}}\right|^{2 a}$ associated with the vertex which is counted in $V_{2 ; a}=1$ is necessarily integrated. Hence the analysis of the rows IV, V, and VI are completely similar to what we have done for the rows I, II, and III, respectively. As a last remark, we stress that there are no corrections to the wave function $Z_{b}$ because all amplitudes at first order are already convergent. Hence we can put the coupling $Z_{b}$ to 0 .

In conclusion,

- the expansion of marginal 4-point functions around their local part gives a log-divergent terms which renormalize the coupling constants $\eta_{+}$or $\lambda$.

- The expansion of $\frac{D}{2}$-divergent or log-divergent 2-point graphs around their local parts yield a $\frac{D}{2}$-divergent or log-divergent term renormalizing either the mass or $Z_{a}$.

- All remainders are convergent and will bring enough decay for ensuring the final summability over scale attributions. From this point, the procedure for performing this last sum over attributions is standard and will secure the renormalization at all orders of perturbation theory according to techniques developed in [68]. Thus, Theorem 1 holds.

\section{A RANK $\boldsymbol{d}=3$ RENORMALIZABLE MODEL $\times$}

We adopt the same strategy as in Sec. VI, to prove the renormalizability of a model $\times$. After listing its primitively divergent connected graphs, we proceed with the renormalization procedure. The same conditions $C_{\partial \mathcal{G}} \geq 1$ and $N_{\text {ext }}$ even must be true. 


\section{A. List of divergent graphs}

We are interested in a rank $d=3$ model $\times$ with $a=\frac{1}{2}, b=1$, and $D=1$. This choice $b=1$ gives a Laplacian in the kinetic term and an integer power of the interaction $|p| \phi^{4}$. The interactions are of the same form as given in Fig. 5 with the sole difference that we enhance by a product of $|p|$ on both edges in the melonic interaction.

We start from (77) and (78), the superficial degree of divergence for generic graphs

$$
\begin{gathered}
\omega_{\mathrm{d} ; \times \times}\left(\mathcal{G}^{\text {melon }}\right) \leq-\left(C_{\partial \mathcal{G}}-1\right)-\frac{1}{2}\left(2 N_{\mathrm{ext}}-4\right)-2 V_{2}-V_{2 ; a}-\Delta_{\times}^{\text {melon }}, \\
\omega_{\mathrm{d} ; \times}\left(\mathcal{G}^{\text {non-melon }}\right) \leq-1-\left(C_{\partial \mathcal{G}}-1\right)-\frac{1}{2}\left(N_{\mathrm{ext}}-4\right)-2 V_{2}-V_{2 ; a}-\Delta_{\times}^{\text {non-melon }} .
\end{gathered}
$$

We observe that both counter-terms $C T_{2 ; 2 a}$ and $C T_{2 ; b}$ disappear from the power counting. As degree-2 vertices, they are neutral for the power counting.

Our previous analysis shows that, for any $N_{\text {ext }} \geq 4$, the amplitude is convergent. We concentrate on the remaining case $N_{\text {ext }}=2$. From Lemma 4, we can still combine the analysis of $V_{(4)}=1$ and $V_{(4)}>1$ at $N_{\text {ext }}=2$ and have

$$
\begin{aligned}
& \omega_{\mathrm{d} ; \times}\left(\mathcal{G}^{\text {melon }}\right) \leq-\left(C_{\partial \mathcal{G}}-1\right)-2 V_{2}-V_{2 ; a}-\Delta_{\times}^{\text {melon }} \leq 0, \\
& \omega_{\mathrm{d} ; \times}\left(\mathcal{G}^{\text {non-melon }}\right) \leq-\left(C_{\partial \mathcal{G}}-1\right)-2 V_{2}-V_{2 ; a}-\Delta_{\times}^{\text {non-melon }} \leq 0 .
\end{aligned}
$$

The only way to achieve logarithmic divergence is to have exactly $C_{\partial \mathcal{G}}=1, V_{2}=0=V_{2 ; a}$. For a non-melonic graph, we further impose $\Delta_{\times}^{\text {non-melon }}=0$ from which we infer $\rho_{\times}=2 V_{(4)}-1$. Knowing that $\rho_{\times} \leq 2 V_{\times ; 4}$, this leads us to $\left(\rho_{\times}=2 V_{\times ; 4}-1 ; V_{4}=0\right)$. The case of a melonic graph yields $\Delta_{\times}^{\text {melon }}=0$ which gives $\rho_{\times}=2 V_{(4)}-2$, which together with $\rho_{\times} \leq 2 V_{\times ; 4}$ yields two possibilities: either $\left(\rho_{\times}=2 V_{\times ; 4} ; V_{4}=1\right)$ or $\left(\rho_{\times}=2 V_{\times ; 4}-2 ; V_{4}=0\right)$.

We have the following proposition.

Proposition 2 (List of primitively divergent graphs for model $\times$ ). The $p^{2 a} \phi^{4}$-model $\times$ with parameters $D=1, d=3, a=\frac{1}{2}, b=1$ has the following primitively divergent graphs which obey $\left(\Omega(\mathcal{G})=\omega\left(\mathcal{G}_{\text {color }}\right)-\omega(\partial \mathcal{G})\right)$. See Table V.

In Appendix C, we have illustrated an infinite family of 2-point graphs with log-divergent amplitudes; see Figs. 9-11. Thus, this theory is not super-renormalizable in the usual sense because it possesses an infinite family of corrections to the mass, $Z_{a}$ and $Z_{2 a}$ couplings. It does not also fit the definition of a just-renormalizable theory because all corrections of the coupling $\lambda$ and $\eta_{\times}$are finite. Again this is a specific feature brought by the enhancement of non-local tensor interactions.

With the above analysis, we can now prove that the following theorem holds.

Theorem 2. The $p^{2 a} \phi^{4}$ model $\times$ with parameters $D=1, d=3, a=\frac{1}{2}, b=1$, with action defined by (2) is renormalizable at all orders of perturbation.

Let us address a few comments about the topology associated with the divergent diagrams in the models studied so far (including those of the model + ). All divergent melonic diagrams, with $\Omega(\mathcal{G})=0$, corresponds to spheres. Inspecting the case $\Omega(\mathcal{G})>0$, the question whether the graphs are spheres can be simply addressed in rank $d=3$. Indeed, the rank 3 case has been investigated by Ryan, ${ }^{70}$ and as a corollary, one claims that all planar diagrams which contain one (Heegaard) embedding which is also

TABLE V. List of primitively divergent graphs of the $p^{2 a} \phi^{4}$-model $\times$.

\begin{tabular}{lcccccccc}
\hline \hline $\mathcal{G}$ & $N_{\text {ext }}$ & $V_{2}$ & $V_{2 ; a}$ & $V_{4}$ & $\rho_{\times}$ & $C_{\partial \mathcal{G}}-1$ & $\Omega(\mathcal{G})$ & $\omega_{\mathrm{d} ; \times}(\mathcal{G})$ \\
\hline I & 2 & 0 & 0 & 0 & $2 V_{\times ; 4}-1$ & 0 & 1 & 0 \\
II & 2 & 0 & 0 & 0 & $2 V_{\times ; 4}-2$ & 0 & 0 & 0 \\
III & 2 & 0 & 0 & 1 & $2 V_{\times ; 4}$ & 0 & 0 & 0 \\
\hline \hline
\end{tabular}


a planar jacket, are spheres. Hence, we will restrict to that rank. All diagrams of Table IV restricted at $d=3$ and Table $\mathrm{V}$ (which is at rank $d=3$ ) can be now studied: $\Omega(\mathcal{G})=0$ is trivial; $\Omega(\mathcal{G})=1$ and $N_{\text {ext }}=2$ impliy that there is an embedding with 0 genus (the boundary graph of such diagrams is always the sunset planar graph). Thus, they are all spheres. Let us concentrate on the only remaining case $N_{\text {ext }}=4$ of Table IV. One must know that the boundary graphs of bi-partite diagrams are also bi-partite. Therefore there is a unique possibility for a connected boundary graph made with $N_{\text {ext }}=4$ fields. It is easy to check that this boundary graph is again planar. We come to $\Omega(\mathcal{G})=\omega\left(\mathcal{G}_{\text {color }}\right)=1$, which implies that there is a planar jacket (in fact two). We conclude once more that the divergent diagram with $N_{\text {ext }}=4$ are also spheres. Hence, all divergent contributions in the renormalization analysis in rank $d=3$ are spheres. For higher rank $d>3$, nothing can be stated without further analysis.

\section{B. Renormalization}

We follow a similar scheme as developed in Sec. VI B. We sketch the expansion of amplitudes of the graphs listed in Table $\mathrm{V}$ and check that their local parts indeed take the form of the terms in the Lagrangian of the model $\times$ of Sec. VII A. Doing a Taylor expansion of the amplitudes, we will also show that the subleading orders are convergent.

\section{Renormalization of divergent 2-point functions}

In the model $\times$, we only have log-divergent 2-point graphs as listed in Table V. For type I and II graphs, note that $\rho_{\times}<2 V_{\times ; 4}$, and therefore one or two edges of their boundary graph are touched by external faces which are enhanced. This entails that the boundaries of these graphs are equipped with $\left|p_{f[1]}^{\text {ext }}\right|^{2 a}$ and $\left|p_{f[1]}^{\text {ext }}\right|^{4 a}$ and therefore of the form of $C T_{2 ; a}, C T_{2 ; 2 a}$, respectively. On the other hand, for a log-divergent graph of the type III, we have $\rho_{\times}=2 V_{\times ; 4}$ and its boundary graph does not have any enhanced edges and so takes the form of the mass term.

In the following, we only address the 2-point graphs of type I and II and will give the main points leading to the treatment of type III graphs.

Let us consider the amplitude $A_{2}\left(\left\{p_{f}^{\text {ext }}\right\}\right)$ of 2-point non-melonic and melonic graphs obeying, respectively, the rows I and II of Table V. These graphs have 3 external faces labeled by $f \in \mathcal{F}_{\text {ext }}$ $=\left\{f_{[1]}, f_{2}, f_{3}\right\}$, with an enhanced color 1 strand. By an argument of symmetry, our following study will give the same result for a graph with another enhanced color.

We perform a Taylor expansion of external face factors as given in (100) and the amplitude $A_{2}\left(\left\{p_{f}^{\text {ext }}\right\}\right)$ takes a similar form as (106); we replace $\kappa\left(\eta_{+}\right)$with $\kappa\left(\eta_{\times}\right)$and have an extra term $\left|p_{f_{[1]}}^{\text {ext }}\right|^{2 \xi}$ present, where $\xi=a$ for the type I and $\xi=2 a$ for type II graph. Then the 0th order term in the expansion in $R_{f}$ expresses as

$$
\begin{aligned}
& A_{2}\left(\left\{p_{f}^{\mathrm{ext}}\right\} ; 0\right)=\kappa\left(\eta_{\times}\right)\left[\int\left[\prod_{l_{\mathrm{ext}}} d \alpha_{l_{\mathrm{ext}}}\right]\left|p_{f_{[1]}^{\mathrm{ext}}}\right|^{2 \xi}\right. \\
& \times e^{-\alpha_{l_{\text {ext } 1}} \sum_{\xi}\left(\left.\left|p_{f_{[1]}}^{\text {ext }} 2^{2 \xi}+\right| p_{f_{2}}^{\text {ext }}\right|^{2 \xi}+\left|p_{f_{3}}^{\text {ext }}\right|^{2 \xi}+\mu\right)} e^{-\alpha_{\text {ext } 2} \sum_{\xi}\left(\left|p_{f_{[1]}}^{\text {ext }}\right|^{2 \xi}+\left|p_{f_{2}}^{\text {ext }}\right|^{2 \xi}+\left|p_{f_{3}}^{\text {ext }}\right|^{2 \xi}+\mu\right)} \\
& \times\left[\sum_{p_{f}} \int\left[\prod_{\ell} d \alpha_{\ell} e^{-\alpha_{\ell} \mu}\right] \prod_{f \in \mathcal{F}_{\text {int }}}\left[e^{-\left(\sum_{\ell} \alpha_{\ell}\right) \sum_{\xi}\left|p_{f}\right|^{2 \xi}}\right]\left[\prod_{s=1}^{d} \prod_{v_{s} \in \mathcal{V}_{x ; 4 ; s}}\left(\epsilon \tilde{p}^{2 a}\right)_{v_{s}}\right]\right] .
\end{aligned}
$$

It is explicit here from the pattern of the external data that this amplitude takes the form of the $C T_{2 ; \xi}$ term. We identify then the factor associated with the internal data as having a degree of divergence $\omega_{\mathrm{d} ; \times}=0$, given by our power counting analysis in Sec. VII A. Adding all colored symmetric contribution with respect to $s$ of this graph, the sum of these amplitudes renormalizes the coupling $Z_{\xi}$.

We now treat the higher orders in the Taylor expansion in the form $\sum_{f} R_{f}$ of $A_{2}\left(\left\{p_{f}^{\text {ext }}\right\}\right)$. The first order remainder involving the sum $\sum_{f} R_{f}$ can be bounded in the same vein as (108) and we find 


$$
\left|R_{2}\right| \leq K \prod_{(i, k)} M^{-2 b\left(i\left(G_{k}^{i}\right)-e\left(G_{k}^{i}\right)\right)} M^{\omega_{\mathrm{d} ;}\left(G_{k}^{i}\right)=0},
$$

for some constant $K$. We are guaranteed of the convergence of this remainder, and that all higher order remainders can be shown more convergent. We are ensured about the summability over scale attributions in this case.

For the log-divergent graphs III, the analysis is similar except that we do not have extra external momenta contributions $\left|p_{f_{[1]}}^{\text {ext }}\right|^{2 \xi}$ appearing, as discussed earlier.

We conclude that,

- the expansion of log-divergent 2-point graphs around their local parts yields log-divergent terms renormalizing $Z_{a}, Z_{2 a}$, and the mass.

- All remainders are convergent and bring a sufficient decay for ensuring the summability over scale attribution. This means that renormalization at all orders of perturbation theory according to [68] can be achieved. Therefore, we conclude that Theorem 2 holds.

- There is no wave function renormalization associated with $Z_{b}$ for the model $\times$ because all amplitudes at the leading order are already convergent.

Without much work, we observe that several other models listed in Table III are renormalizable at $d=3$, just like the present model is [up to a change of (109), (110), (111), Table V, and (113)]. More surprising is perhaps, at fix $D$, we even suspect that it might have a continuum of renormalizable theories for a range of values of $b$.

\section{CONCLUSION}

We have addressed the UV perturbative (at all orders) multi-scale renormalization analysis of the so-called enhanced quartic melonic tensor field theory at any rank $d$ of the tensor fields and for any group $\left(U(1)^{D}\right)^{d}$. Studied in the momentum space, the models are endowed with powers of momenta in the interaction terms which are roughly of the form $p^{2 a} \phi^{4}, a>0$, and which might be associated with derivative couplings. The case $a=0$ being well-studied in the literature can be recovered at this limit. Through the enhancing procedure, amplitudes which were suppressed in models at $a=0$ now participate in the analysis at $a>0$.

In order to achieve renormalizability in enhanced models, the propagators need a more general form than the usual Laplacian dynamics: we thus assume the propagators to be of the form $\left(\sum_{\xi}|p|^{2 \xi}+\mu\right)^{-1}$, with $\xi=a, 2 a, b$ strictly positive. Two types of models were introduced and studied in parallel in this work: an asymmetric model + and a symmetric model $\times$. From the multi-scale analyses of these models, we identify new combinatorial quantities which allow us to write down a power counting theorem in terms of quasi-local subgraphs. At any rank $d>2$, group dimension $D \geq 1$, we have found intervals of values for $a$ and $b$ for which both models are potentially renormalizable at all orders of perturbation theory. Let us give a summary of the particularities of each model.

For arbitrary $d$ and $D$, which specify the rest of the parameters, we find a two-dimensional grid $((\mathbb{N}-\{0,1,2\}) \times(\mathbb{N}-\{0\}))$ of just-renormalizable models + . As expected, the amplitudes of nonmelonic diagrams start to contribute to the flow and dominate the melonic amplitudes in each model. In fact, all the 4-point melonic diagrams become convergent. We found that the enhanced coupling $\eta_{+}$will contribute to the flow of the melonic coupling $\lambda$, whereas the opposite is not possible. Introducing enhanced interactions of $\operatorname{Tr}_{4}\left(p^{2 a} \phi^{4}\right)$ influences the study of the 2-point function as it requires to introduce a particular counter-term of the form $\operatorname{Tr}_{2}\left(p^{2 a} \phi^{2}\right)$.

The model $\times$ is also renormalizable for a tuned set of parameters. Again, melonic and nonmelonic amplitudes can be of the same divergence degree, as expected from the enhanced interactions. However, something puzzling happens in this model: all 4-point amplitudes prove to be finite and only 2-point amplitudes may diverge. There are infinitely many log-divergent 2-point amplitudes: the bound (which saturates) for the degree of divergence for these graphs can be made independent of the number of $\phi^{4}$ (of any type) interaction vertices. In this sense, the model $\times$ is not strictly a just-renormalizable theory, but not just simply a super-renormalizable theory in the standard sense. The detailed study of the 2-point graphs imposes us to include two counter-terms for removing all divergences: $\operatorname{Tr}_{2}\left(p^{2 a} \phi^{2}\right)$ and $\operatorname{Tr}_{2}\left(p^{4 a} \phi^{2}\right)$. Thus, the flow of this model is uniquely 
driven by 2-point functions. We conjecture that there are several other renormalizable models of this kind.

In this work, we have studied models with $\phi^{4}$ melonic bubble interactions. In any of these interactions, weak edges are clearly unique. If one is interested in higher order interactions $\phi^{n}$ with $n>4$, the techniques used here to analyze the perturbative renormalizability of models can be used. However, the following issue arises: since $\phi^{n \geq 6}$ bubbles may have several weak links, and even of different number of strands depending on the rank $d$, there is a number of possibilities to enhance those weak edges (which ones and by which power of momenta). It becomes obvious that the question of renormalizability in such enhanced models is much more involved.

We recall that enhanced tensor models are introduced from attempts to escape the branched polymer phase of the colored tensor models. If enhanced tensor models are turned into field theories, then the large $N$-limit becomes the UV-limit (large $p$ ). As far as the present study is concerned, the perturbative renormalizability of the enhanced models of the type presented here might not immediately tell us anything about new limits or new phases of these enhanced models. Having renormalizability rather ensures us that the field theory counterparts of these models are long-lived, defined through several layers of momentum scales, and might be UV-complete. This is definitely an important and encouraging point to keep up with their study.

Another important aim that could be certainly reached from our analysis is the computation of the perturbative $\beta$-functions for these models. There are several arguments putting forward that ordinary $\phi^{4}$ tensor field theories are perturbatively asymptotically free..$^{27,47}$ The main ingredient leading to asymptotic freedom is the presence of a wave-function renormalization which dominates the renormalized coupling constant. Nevertheless, the models addressed in this paper seem to belong to another class, simply because of the presence of the several couplings $Z_{\xi}, \xi=a, 2 a$, and the fact that $Z_{b}$ does not get any radiative corrections. For the model + , we realize that the RG equations might be more involved than one might think because of the number of couplings in the theory. Thus, only careful computations of the $\beta$-functions of this model could help to understand the UV-behavior of the model + .

Beyond perturbation, non-perturbative properties of these models can be sought in the future. In particular, the next steps of the program for enhanced models would be to find UV and IR fixed points which may exist and, from these, perhaps complete trajectories from the UV to the IR. The proof of the perturbative renormalizability is again encouraging for this next level. The FRG approach has been applied to ordinary tensor field theories with interesting results. Extending the methods to the enhanced theory space is again to be done. In particular, if one shows the existence of stable IR fixed points in enhanced tensor field theories, it could give them a firmer underpinning as interesting candidates undergoing a phase transition from discrete-like geometries to some condensate-like geometry.

\section{ACKNOWLEDGMENTS}

The research of R.T. is supported by the Netherlands Organisation for Scientific Research (NWO) within the Foundation for Fundamental Research on Matter (FOM) Grant No. 13VP12. R.T. thanks the Max Planck Institute for Gravitational Physics and Potsdam-Golm (Albert Einstein Institute) for their hospitality while this work was in progress. J.B.G. thanks the Laboratoire de Physique Théorique d'Orsay, Université Paris 11, for its hospitality.

\section{APPENDIX A: SPECTRAL SUMS}

In this appendix, we perform spectral sums over internal momenta. We start from a basic sum and will go for more involved cases in the dimension $D$. In the following, we use $B>0$ as a parameter, and in the text, $B=M^{-i_{l}}$. Targeting upper bounds on $B^{\alpha}$, we focus on the terms with $\alpha<0$.

Noting that for constants $B>0, a>0$, and $b>0$, a single sum over a $p_{s ; l}$ behaves like

$$
\sum_{p=1}^{\infty} p^{a} e^{-B p^{b}}=k B^{-\frac{(a+1)}{b}}\left(1+O\left(B^{\frac{(a+1)}{b}}\right)\right),
$$


where $k$ is an $a$-dependent constant. This relation has been proved for instance in Appendix A of Ref. 47. This sum can be generalized as

$$
\begin{aligned}
& \sum_{p=1}^{\infty} p^{a} e^{-B\left(p^{b}+p^{c}\right)}=\sum_{n=0}^{\infty} \frac{(-B)^{n}}{n !} \sum_{p=1}^{\infty} p^{a+c n} e^{-B p^{b}} \\
& =k \sum_{n=0}^{\infty} \frac{(-B)^{n}}{n !} B^{-\frac{(a+c n+1)}{b}}\left(1+O\left(B^{\frac{(a+c n+1)}{b}}\right)\right) \\
& =k B^{-\frac{(a+1)}{b}} e^{-B^{1-\frac{c}{b}}}\left(1+O\left(B^{\frac{(a+1)}{b}}\right)+B^{\left(1-\frac{c}{b}\right)} O\left(B^{\frac{(a+c+1)}{b}}\right)+B^{2\left(1-\frac{c}{b}\right)} O\left(B^{\frac{(a+2 c+1)}{b}}\right)\right) \\
& =k B^{-\frac{(a+1)}{b}} e^{-B^{1-\frac{c}{b}}}\left(1+O\left(B^{\frac{(a+1)}{b}}\right)\right),
\end{aligned}
$$

where we use (A1) at an intermediate step. For the specific choice $c \leq b$, such that $1-\frac{c}{b} \geq 0$, the previous result recasts as

$$
\sum_{p=1}^{\infty} p^{a} e^{-B\left(p^{b}+p^{c}\right)}=k B^{-\frac{(a+1)}{b}}\left(1+O\left(B^{1-\frac{c}{b}}\right)\right) .
$$

It is not difficult then to use the same routine and get, for $c+d \leq 2 b$,

$$
\begin{aligned}
& \sum_{p=1}^{\infty} p^{a} e^{-B\left(p^{b}+p^{c}+p^{d}\right)}=\sum_{m, n=0}^{\infty} \frac{(-B)^{n+m}}{n ! m !} \sum_{p=1}^{\infty} p^{a+c n+d m} e^{-B p^{b}} \\
& =k \sum_{m, n=0}^{\infty} \frac{(-B)^{n+m}}{n ! m !} B^{-\frac{(a+c n+d m+1)}{b}}\left(1+O\left(B^{\frac{(a+c n+d m+1)}{b}}\right)\right) \\
& =k B^{-\frac{(a+1)}{b}} e^{-B^{2-\frac{(c+d)}{b}}}\left(1+O\left(B^{\frac{(a+1)}{b}}\right)\right)=k B^{-\frac{(a+1)}{b}}\left(1+O\left(B^{2-\frac{(c+d)}{b}}\right)\right) .
\end{aligned}
$$

Note that if the above calculations were approximated at $c \leq b$, using $e^{-B\left(p^{b}+p^{c}\right)} \leq e^{-2 B p^{c}}$ and, if $c \leq$ $d \leq b, e^{-B\left(p^{b}+p^{c}+p^{d}\right)} \leq e^{-3 B p^{c}}$, the sum behavior could change [using (A1) with a modified $B$ ]. Hence using this bound is not the optimal choice.

We will need a companion sum in the dimension $D$

$$
\begin{aligned}
& \sum_{p_{1}, \ldots, p_{D}=1}^{\infty}\left(\sum_{l=1}^{D} p_{l}^{a}\right)^{n} e^{-B\left(\sum_{l=1}^{D} p_{l}^{b}\right)}=\sum_{\sum_{i} n_{i}=n} \frac{n !}{\prod_{l=1}^{D} n_{l} !} \sum_{p_{1}, \ldots, p_{D}=1}^{\infty} \prod_{l=1}^{D} p_{l}^{a n_{l}} e^{-B p_{l}^{b}} \\
= & c^{\prime} \sum_{\sum_{i} n_{i}=n} \frac{n !}{\prod_{l=1}^{D} n_{l} !} B^{-\frac{\sum_{l}\left(a n_{l}+1\right)}{b}} \prod_{l=1}^{D}\left(1+O\left(B^{\frac{\left(a n_{l}+1\right)}{b}}\right)\right)=c^{\prime} B^{-\frac{(a n+D)}{b}}\left(1+O\left(B^{\frac{1}{b}}\right)\right),
\end{aligned}
$$

where $c^{\prime}=c^{D} 2^{n}$. We extend this computation, in the case of multiple powers in the exponential, with $c \leq b$

$$
\begin{aligned}
& \sum_{p_{1}, \ldots, p_{D}=1}^{\infty}\left(\sum_{l=1}^{D} p_{l}^{a}\right)^{n} e^{-B \sum_{l=1}^{D}\left(p_{l}^{b}+p_{l}^{c}\right)}= \\
& c^{\prime} \sum_{\sum_{i} n_{i}=n} \frac{n !}{\prod_{l=1}^{D} n_{l} !} B^{-\frac{\sum_{l}\left(a n_{l}+1\right)}{b}} e^{-B^{1-\frac{c}{b}}} \prod_{l=1}^{D}\left(1+O\left(B^{\frac{\left(a n_{l}+1\right)}{b}}\right)\right. \\
&=c^{\prime} B^{-\frac{(a n+D)}{b}} e^{-B^{1-\frac{c}{b}}}\left(1+O\left(B^{\frac{1}{b}}\right)\right)=c^{\prime} B^{-\frac{(a n+D)}{b}}\left(1+O\left(B^{\frac{1}{b}}\right)+O\left(B^{1-\frac{c}{b}}\right)\right),
\end{aligned}
$$

where (A2) and (A3) have been used. Depending on $b-c \leq 1$ or otherwise, the two big-O functions can be reduced into one. However, being interested in the leading order, this relation is sufficient to 
proceed further. Using the same techniques, the last useful sum evaluates as

$$
\begin{aligned}
& \sum_{p_{1}, \ldots, p_{D}=1}^{\infty}\left(\sum_{l=1}^{D} p_{l}^{a}\right)^{n} e^{-B\left(\sum_{l=1}^{D}\left(p_{l}^{b}+p_{l}^{c}+p_{l}^{d}\right)\right)}=c^{\prime} B^{-\frac{(a n+D)}{b}} e^{-B^{2-\frac{(c+d)}{b}}}\left(1+O\left(B^{\frac{1}{b}}\right)\right) \\
= & c^{\prime} B^{-\frac{(a n+D)}{b}}\left(1+O\left(B^{\frac{1}{b}}\right)+O\left(B^{2-\frac{(c+d)}{b}}\right)\right),
\end{aligned}
$$

where the last equality is obtained for $c+d \leq 2 b$.

\section{APPENDIX B: DIVERGENCES IN MODEL $+\left(d=3, D=1, a=\frac{1}{2}, b=\frac{3}{4}\right)$}

We consider here a specific model + with parameter given as $d=3, D=1, a=\frac{1}{2}, b=\frac{3}{4}$. The mass term and interactions are of the form given in Fig. 5.

In this appendix, we illustrate graphs which satisfy the power counting achieved in Table IV in Sec. VI A.

The superficial degree of divergence of a graph $\mathcal{G}$ is given in (44) that we specialize for $D=1$, $a=\frac{1}{2}$, and $b=\frac{3}{4}$ as

$$
\omega_{\mathrm{d} ;+}(\mathcal{G})=-\frac{3}{2} L+F_{\text {int }}+\rho_{+}+\rho_{2 ; a}+\frac{3}{2} \rho_{2 ; b} .
$$

We use the bipartite colored graph representation of the Feynman graphs of the model. Edges which are dashed are propagators; edges in the interaction vertex can be in bold or not. If they are in bold that means that they receive an enhancement factor of $p^{2 a}$. The figures illustrating graphs have red lines that facilitate the identification of the face structure of the graphs. Given a colored graph, we emphasize a red cycle (made with alternating edges and dashed edges with red color) that indicates a particular closed face. Naturally, this face will be the source of an enhanced power counting if it contains a bold edge.

We only list here some divergent graphs contributing to the renormalization of the interactions.

(i) We consider 2-point functions, $N_{\text {ext }}=2$.

(i1) We consider $V_{(4)}=1$ or divergent tadpoles given in Fig. 6. The graphs a and $\mathrm{b}$ are melonic with $V_{(4)}=1, \rho_{+}+\rho_{2 ; a}+\frac{3}{2} \rho_{2 ; b}=0$, and $\omega_{\mathrm{d} ;+}=\frac{1}{2}$, whereas the graph $\mathrm{c}$ is non-melonic with $V_{+; 4}=1, \rho_{+}=1, \rho_{2 ; a}+\frac{3}{2} \rho_{2 ; b}=0$, and $\omega_{\mathrm{d} ;+}=\frac{1}{2}$. The graphs $\mathrm{d}$ and e with $V_{(4)}=1$ and $V_{2 ; a}=1 \rho_{+}+\frac{3}{2} \rho_{2 ; b}=0, \rho_{2 ; a}=1$ are melonic and log-divergent with $\omega_{\mathrm{d} ;+}=0$; the graph $\mathrm{f}$ is non-melonic with $V_{+; 4}=1, V_{2 ; a}=1, \rho_{+}=1=\rho_{2 ; a}, \rho_{2 ; b}=0$ and also log-divergent with $\omega_{\mathrm{d} ;+}=0$.

(i2) We consider $V_{(4)}=2$ [in particular, $\left(V_{4}, V_{+; 4}\right)=(1,1)$ or $(0,2)$ ] and its generalizations given in Fig. 7. Note that if we increase $V_{+; 4}$ in the way of producing c $\left(V_{+; 4}=3\right.$
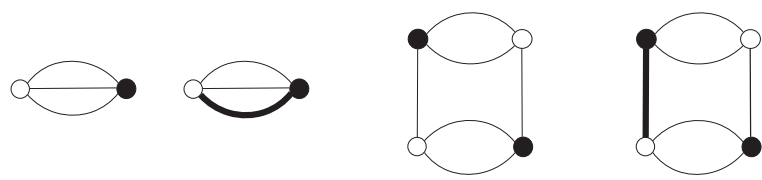

FIG. 5. Rank $d=3$, mass, enhanced $\phi^{2}$-interaction, and (simple and enhanced) $\phi^{4}$-interactions.

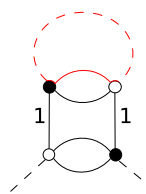

a

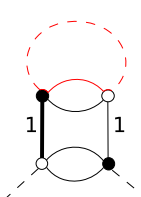

b

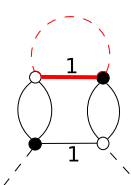

C

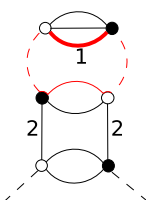

d

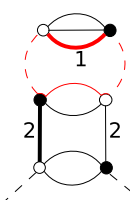

e

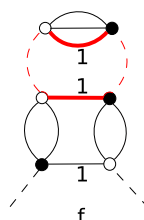

$\mathrm{f}$

FIG. 6. Divergent graphs with $N_{\text {ext }}=2$ and $V_{(4)}=1$. 


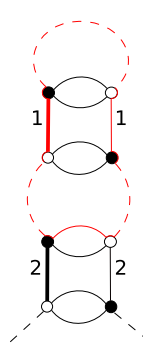

a

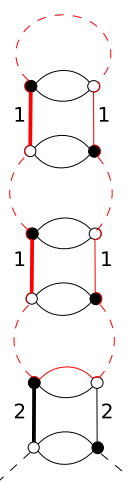

b

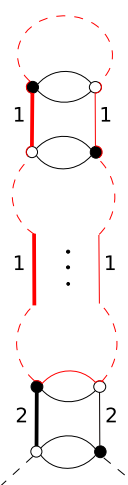

C

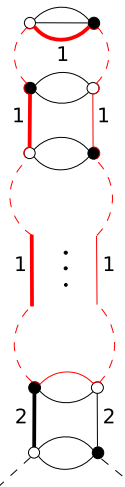

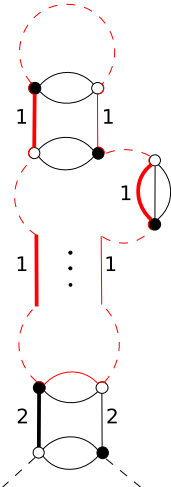

FIG. 7. A family of melonic graphs [(a)-(c) $]$ with $N_{\text {ext }}=2$ and $\omega_{\mathrm{d} ;+}=\frac{1}{2}$ and a family of log-divergent melonic graphs [(d) and (e)] with $N_{\text {ext }}=2$.

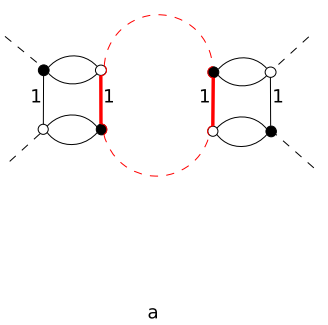

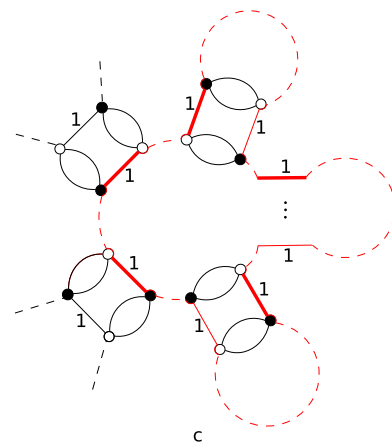

c

FIG. 8. A family of log-divergent non-melonic graphs with $N_{\text {ext }}=4$ and $V_{+; 4} \geq 2$.

given by the graph b), then we have for arbitrary $V_{+; 4}, L=1+2\left(V_{(4)}-1\right)=2 V_{(4)}-1$, $F_{\text {int }}=2 V_{(4)}$, and $\rho_{+}=V_{(4)}-1, \rho_{2 ; a}=0$, and $\rho_{2 ; b}=0$, therefore $\omega_{\mathrm{d} ;+}=\frac{1}{2}$ and is independent of $V_{(4)}$ (or $\left.V_{+; 4}\right)$ which is expected. To these graphs, we can add the enhanced 2-point function $V_{2 ; a}=1$ to any of the internal lines as illustrated in the graphs $\mathrm{d}$ and e. We have for arbitrary $V_{+; 4}$ and $V_{2 ; a}=1, L=2 V_{(4)}, F_{\text {int }}=2 V_{(4)}, \rho_{+}=V_{(4)}-1, \rho_{2 ; a}=1$, and $\rho_{2 ; b}=1$ therefore $\omega_{\mathrm{d} ;+}=0$ is independent of $V_{(4)}\left(\right.$ or $\left.V_{+; 4}\right)$.

(ii) We now consider 4-point functions, $N_{\text {ext }}=4$. First, set $V_{+; 4}=2$. The non-melonic graph (a) of Fig. 8 is logarithmic-divergent since $L=2, F_{\text {int }}=1, \rho_{+}=2, \rho_{2 ; a}=0$, and $\rho_{2 ; b}=0$. This graph generalizes to (b) and then to (c) such that $F_{\text {int }}=1+d^{-}\left(V_{+; 4}-\frac{N_{\text {ext }}}{2}\right)=-3+2 V_{+; 4}, \rho_{+}=V_{+; 4}$, $\rho_{2 ; a}=0$, and $\rho_{2 ; b}=0$.

\section{APPENDIX C: DIVERGENCES IN MODEL $\times\left(d=3, D=1, a=\frac{1}{2}, b=1\right)$}

We illustrate some divergent amplitudes in the model $\times$ with parameters given above. We keep the same meaning of the graphical representation for graphs as in Appendix B.

The superficial degree of divergence of a graph $\mathcal{G}$ has been given in Ref. 47 and that we evaluate at $D=1, a=\frac{1}{2}$, and $b=1$ as

$$
\omega_{\mathrm{d} ; \times}(\mathcal{G})=-2 L+F_{\mathrm{int}}+\rho_{\times}+\rho_{2 ; a}+2 \rho_{2 ; b} .
$$

We consider 2-point functions, $N_{\mathrm{ext}}=2$.

(1) We consider tadpole graphs with $V_{(4)}=1$ given in Fig. 9 which are log-divergent. The graphs (a) and (b) are melonic whereas the graph (c) is non-melonic. 


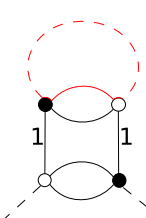

a

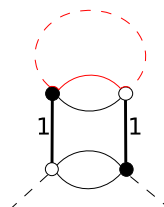

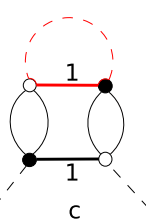

C

FIG. 9. Log-divergent graphs with $N_{\text {ext }}=2$ and $V_{(4)}=1$.

(2) Consider now $V_{(4)} \geq 2$. Melonic graphs (respectively, non-melonic graphs) with $V_{(4)}=2$ and their generalizations for $V_{(4)}>2$ are given in Fig. 10 (respectively, Fig. 11).

First, focus on the melonic graphs of Fig. 10. The melonic graphs, (a) and (d) with $N_{\text {ext }}=2$ and $V_{(4)}=2$ (but $V_{\times ; 4} \geq 1$ ) give $\omega_{\mathrm{d} ; \times}=0$. Increase $V_{\times ; 4}$ in a way to have the graphs (c) and (f) (intermediate steps are given by graphs (b) and (e), respectively), then we have for arbitrary $V_{\times ; 4}, F_{\text {int }}=2 V_{(4)}, \rho_{\times}=2\left(V_{(4)}-1\right)$, therefore $\omega_{\mathrm{d} ; \times}=0$. With these graphs, we confirm that this model has infinitely many log-divergent 2-point graphs.

Next, consider the non-melonic graphs of Fig. 11. For arbitrary $V_{(4)}=V_{\times ; 4}, F_{\text {int }}=2\left(V_{(4)}\right.$ $-1)+1, \rho_{\times}=1+2\left(V_{(4)}-1\right), \rho_{2 ; a}=0$, and $\rho_{2 ; b}=0$ therefore $\omega_{\mathrm{d} ; \times}=0$. Again, we see that this model has infinitely many graphs that are divergent.

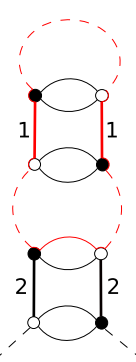

a
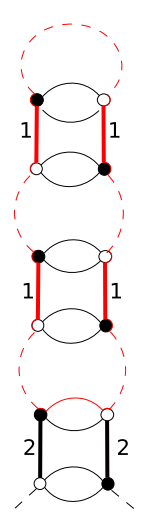

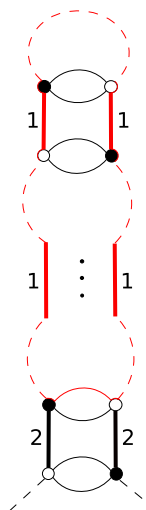

C

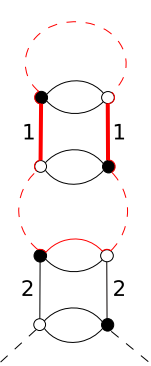

d
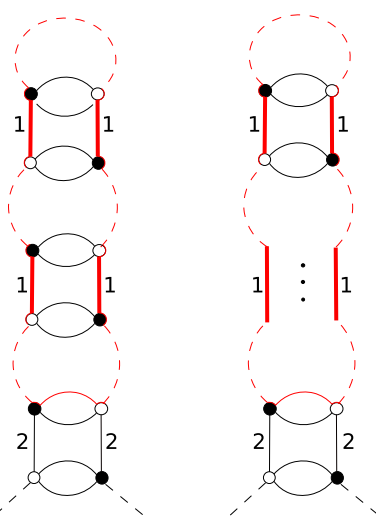

$f$

e

FIG. 10. Melonic graphs with $N_{\mathrm{ext}}=2$ which give $\omega_{\mathrm{d} ; \times}=0$.

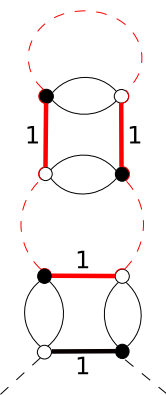

a
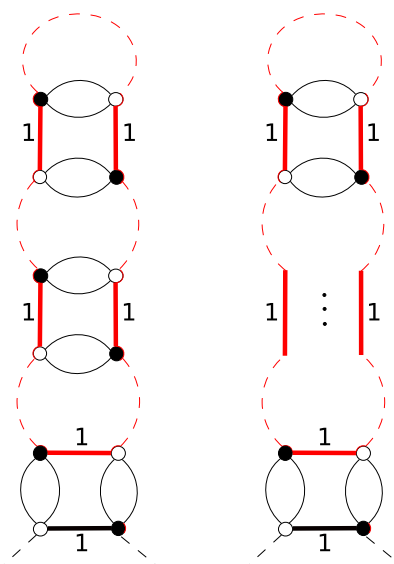

b 年 in . 


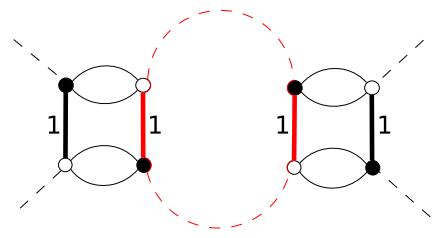

FIG. 12. A convergent non-melonic graph with $N_{\text {ext }}=4$ and $V_{(4)}=V_{\times ; 4}=2$.

(3) A comparison with the previous model shows that the 4-point non-melonic graph with $V_{(4)}=V_{\times ; 4}=2$ of Fig. 12 is convergent $\omega_{\mathrm{d} ; \times}=-1, F_{\text {int }}=1, \rho_{\times}=2, \rho_{2 ; a}=0$, and $\rho_{2 ; b}=0$.

${ }^{1}$ J. Ambjorn, B. Durhuus, and T. Jonsson, "Three-dimensional simplicial quantum gravity and generalized matrix models," Mod. Phys. Lett. A 6, 1133 (1991).

${ }^{2}$ M. Gross, "Tensor models and simplicial quantum gravity in >2-D," Nucl. Phys. Proc. Suppl. 25A, 144 (1992).

${ }^{3}$ N. Sasakura, "Tensor model for gravity and orientability of manifold," Mod. Phys. Lett. A 6, 2613 (1991).

${ }^{4}$ D. V. Boulatov, "A model of three-dimensional lattice gravity," Mod. Phys. Lett. A 7, 1629 (1992); e-print arXiv:hepth/9202074; H. Ooguri, "Topological lattice models in four-dimensions," ibid. 7, 2799 (1992); e-print arXiv:hep-th/9205090.

${ }^{5}$ T. Regge, "General relativity without coordinates," Nuovo Cimento 19(3), 558-571 (1961).

${ }^{6}$ H. Hamber, Quantum Gravitation: The Feynman Path Integral Approach (Springer Publishing, Berlin, New York, 2009), p. 342.

${ }^{7}$ J. Ambjorn, J. Jurkiewicz, and R. Loll, “The Universe from scratch,” Contemp. Phys. 47, 103 (2006); e-print arXiv:hepth/0509010.

${ }^{8}$ J. Ambjorn, A. Goerlich, J. Jurkiewicz, and R. Loll, "Causal dynamical triangulations and the search for a theory of quantum gravity," in Proceedings of the MG13 Meeting on General Relativity (Stockholm University, Sweden, 2012), pp. 120-137; e-print arXiv:1305.6680 [gr-qc].

${ }^{9}$ P. Di Francesco, P. H. Ginsparg, and J. Zinn-Justin, “2-D gravity and random matrices,” Phys. Rep. 254, 1 (1995); e-print arXiv:hep-th/9306153.

${ }^{10}$ G. 't Hooft, "A planar diagram theory for strong interactions," Nucl. Phys. B 72, 461 (1974).

${ }^{11}$ J. Distler and H. Kawai, "Conformal field theory and 2D quantum gravity or who's afraid of Joseph Liouville?," Nucl. Phys. B 321, 509 (1989).

${ }^{12}$ R. Gurau, "The 1/N expansion of colored tensor models," Ann. Henri Poincare 12, 829 (2011); e-print arXiv:1011.2726 [gr-qc].

${ }^{13}$ R. Gurau, "The complete 1/N expansion of colored tensor models in arbitrary dimension," Ann. Henri Poincare 13, 399 (2012); e-print arXiv:1102.5759 [gr-qc].

${ }^{14}$ R. Gurau, "Colored group field theory," Commun. Math. Phys. 304, 69 (2011); e-print arXiv:0907.2582 [hep-th].

${ }^{15}$ R. Gurau, "Lost in translation: Topological singularities in group field theory," Classical Quantum Gravity 27, 235023 (2010); e-print arXiv:1006.0714 [hep-th].

${ }^{16}$ R. Gurau and J. P. Ryan, "Colored tensor models-A review," SIGMA 8, 020 (2012); e-print arXiv:1109.4812 [hep-th].

${ }^{17}$ V. Bonzom, R. Gurau, A. Riello, and V. Rivasseau, "Critical behavior of colored tensor models in the large N limit," Nucl. Phys. B 853, 174 (2011); e-print arXiv:1105.3122 [hep-th].

${ }^{18}$ R. Gurau, "A generalization of the Virasoro algebra to arbitrary dimensions," Nucl. Phys. B 852, 592 (2011); e-print arXiv:1105.6072 [hep-th].

${ }^{19}$ A. Tanasa, "Multi-orientable group field theory," J. Phys. A: Math. Theor. 45, 165401 (2012); e-print arXiv:1109.0694 [math.CO].

${ }^{20}$ V. Bonzom, R. Gurau, and V. Rivasseau, "Random tensor models in the large N limit: Uncoloring the colored tensor models," Phys. Rev. D 85, 084037 (2012); e-print arXiv:1202.3637 [hep-th].

${ }^{21}$ R. Gurau, "The Schwinger Dyson equations and the algebra of constraints of random tensor models at all orders," Nucl. Phys. B 865, 133 (2012); e-print arXiv:1203.4965 [hep-th].

22 R. Gurau, Random Tensors (Oxford University Press, Oxford, 2016).

${ }^{23}$ A. Eichhorn and T. Koslowski, "Continuum limit in matrix models for quantum gravity from the functional renormalization group,” Phys. Rev. D 88, 084016 (2013); e-print arXiv:1309.1690 [gr-qc].

${ }^{24}$ J. Ben Geloun and T. A. Koslowski, "Nontrivial UV behavior of rank-4 tensor field models for quantum gravity," e-print arXiv: 1606.04044 [gr-qc].

${ }^{25}$ A. Eichhorn and T. Koslowski, "Flowing to the continuum in discrete tensor models for quantum gravity," Ann. Inst. H. Poincare Comb. Phys. Interact. 5(2), 173 (2018).

${ }^{26} \mathrm{~S}$. Carrozza and V. Lahoche, "Asymptotic safety in three-dimensional SU(2) group field theory: Evidence in the local potential approximation," Classical Quantum Gravity 34(11), 115004 (2017); e-print arXiv:1612.02452 [hep-th].

${ }^{27}$ V. Rivasseau, "Quantum gravity and renormalization: The tensor track," AIP Conf. Proc. 1444, 18 (2011); e-print arXiv:1112.5104 [hep-th].

${ }^{28} \mathrm{~J}$. Ben Geloun and R. Toriumi, "Parametric representation of rank $d$ tensorial group field theory: Abelian models with kinetic term $\sum_{s}\left|p_{s}\right|+\mu$," J. Math. Phys. 56(9), 093503 (2015); e-print arXiv:1409.0398 [hep-th].

${ }^{29}$ T. Krajewski and R. Toriumi, "Polchinski's exact renormalisation group for tensorial theories: Gaussian universality and power counting,” J. Phys. A: Math. Theor. 49(38), 385401 (2016); e-print arXiv:1511.09084 [gr-qc]. 
${ }^{30}$ T. Krajewski and R. Toriumi, "Exact renormalisation group equations and loop equations for tensor models," SIGMA 12, 068 (2016); e-print arXiv: 1603.00172 [gr-qc].

${ }^{31}$ A. Eichhorn and T. Koslowski, "Towards phase transitions between discrete and continuum quantum spacetime from the renormalization group,” Phys. Rev. D 90(10), 104039 (2014); e-print arXiv:1408.4127 [gr-qc].

${ }^{32}$ D. Benedetti, J. Ben Geloun and D. Oriti, "Functional renormalisation group approach for tensorial group field theory: A rank-3 model," JHEP 1503, 084 (2015); e-print arXiv:1411.3180 [hep-th].

${ }^{33}$ J. Ben Geloun, R. Martini, and D. Oriti, "Functional renormalisation group analysis of tensorial group field theories on $\mathbb{R}^{d}$," Phys. Rev. D 94(2), 024017 (2016); e-print arXiv:1601.08211 [hep-th].

${ }^{34}$ S. Carrozza, V. Lahoche, and D. Oriti, "Renormalizable group field theory beyond melons: An example in rank four," Phys. Rev. D 96(6), 066007 (2017).

${ }^{35}$ R. Gurau and J. P. Ryan, “Melons are branched polymers,” Ann. Henri Poincare 15(11), 2085 (2014); e-print arXiv:1302.4386 [math-ph].

${ }^{36}$ V. Bonzom, "New 1/N expansions in random tensor models," JHEP 1306, 062 (2013); e-print arXiv:1211.1657 [hep-th].

${ }^{37}$ V. Bonzom, T. Delepouve, and V. Rivasseau, "Enhancing non-melonic triangulations: A tensor model mixing melonic and planar maps," Nucl. Phys. B 895, 161 (2015); e-print arXiv:1502.01365 [math-ph].

38 J. Ben Geloun, “A power counting theorem for a $p^{2 a} \phi^{4}$ tensorial group field theory," e-print arXiv:hep-th/1507.00590.

${ }^{39}$ S. Carrozza, Tensorial Methods and Renormalization in Group Field Theories, Springer Theses (Springer, NY, 2014); e-print arXiv:1310.3736 [hep-th].

${ }^{40}$ J. Ben Geloun and V. Rivasseau, "A renormalizable 4-dimensional tensor field theory," Commun. Math. Phys. 318, 69 (2013); e-print arXiv:1111.4997 [hep-th].

${ }^{41}$ J. Ben Geloun and V. Rivasseau, "Addendum to "A renormalizable 4-dimensional tensor field theory," Commun. Math. Phys. 322, 957 (2013); e-print arXiv:1209.4606 [hep-th].

42 J. Ben Geloun and D. O. Samary, "3D tensor field theory: Renormalization and one-loop $\beta$-functions," Ann. Henri Poincare 14, 1599 (2013); e-print arXiv:1201.0176 [hep-th].

${ }^{43} \mathrm{~J}$. Ben Geloun, "Two and four-loop $\beta$-functions of rank 4 renormalizable tensor field theories," Classical Quantum Gravity 29, 235011 (2012); e-print arXiv:1205.5513 [hep-th].

${ }^{44}$ S. Carrozza, D. Oriti, and V. Rivasseau, "Renormalization of tensorial group field theories: Abelian U(1) models in four dimensions," Commun. Math. Phys. 327, 603 (2014); e-print arXiv:1207.6734 [hep-th].

${ }^{45}$ D. O. Samary and F. Vignes-Tourneret, "Just renormalizable TGFT's on $U(1)^{d}$ with gauge invariance," Commun. Math. Phys. 329, 545 (2014); e-print arXiv:1211.2618 [hep-th].

${ }^{46}$ S. Carrozza, D. Oriti, and V. Rivasseau, "Renormalization of a SU(2) tensorial group field theory in three dimensions," Commun. Math. Phys. 330, 581 (2014); e-print arXiv:1303.6772 [hep-th].

${ }^{47}$ J. Ben Geloun, "Renormalizable models in rank $d \geq 2$ tensorial group field theory," Commun. Math. Phys. 332, 117 (2014); e-print arXiv:1306.1201 [hep-th].

${ }^{48}$ S. Carrozza, "Discrete renormalization group for SU(2) tensorial group field theory," Ann. Inst. Henri Poincaré Comb. Phys. Interact. 2, 49-112 (2015); e-print arXiv:1407.4615 [hep-th].

${ }^{49}$ S. Carrozza, "Group field theory in dimension $4-\epsilon$," Phys. Rev. D 91(6), 065023 (2015); e-print arXiv:1411.5385 [hep-th].

${ }^{50}$ V. Rivasseau, "Why are tensor field theories asymptotically free?," Europhys. Lett. 111(6), 60011 (2015); e-print arXiv: 1507.04190 [hep-th].

${ }^{51}$ A. Kegeles and D. Oriti, "Generalized conservation laws in non-local field theories," J. Phys. A: Math. Theor. 49(13), 135401 (2016); e-print arXiv:1506.03320 [hep-th].

${ }^{52}$ A. Kegeles and D. Oriti, "Continuous point symmetries in group field theories," J. Phys. A: Math. Theor. 50(12), 125402 (2017); e-print arXiv:1608.00296 [gr-qc].

53 J. Ben Geloun, "On the finite amplitudes for open graphs in Abelian dynamical colored Boulatov-Ooguri models," J. Phys. A: Math. Theor. 46, 402002 (2013); e-print arXiv:1307.8299 [hep-th].

${ }^{54}$ J. Ben Geloun and S. Ramgoolam, "Tensor models, Kronecker coefficients and permutation centralizer algebras," JHEP 1711, 092 (2017); e-print arXiv:1708.03524 [hep-th].

${ }^{55}$ E. Witten, "An SYK-like model without disorder," e-print arXiv:1610.09758 [hep-th].

${ }^{56}$ R. Gurau, "The complete $1 / N$ expansion of a SYK-like tensor model," Nucl. Phys. B 916, 386 (2017); e-print arXiv:1611.04032 [hep-th].

${ }^{57}$ I. R. Klebanov and G. Tarnopolsky, "Uncolored random tensors, melon diagrams, and the Sachdev-Ye-Kitaev models," Phys. Rev. D 95(4), 046004 (2017); e-print arXiv:1611.08915 [hep-th].

${ }^{58}$ R. Gurau, "Quenched equals annealed at leading order in the colored SYK model," EPL 119(3), 30003 (2017).

${ }^{59}$ V. Bonzom, L. Lionni, and A. Tanasa, "Diagrammatics of a colored SYK model and of an SYK-like tensor model, leading and next-to-leading orders," J. Math. Phys. 58(5), 052301 (2017); e-print arXiv:1702.06944 [hep-th].

${ }^{60}$ S. Sachdev and J. Ye, "Gapless spin fluid ground state in a random, quantum Heisenberg magnet," Phys. Rev. Lett. 70, 3339 (1993); e-print arXiv:cond-mat/9212030.

61 A. Kitaev, http://online.kitp.ucsb.edu/online/entangled15/kitaev/.

62 J. Maldacena and D. Stanford, "Remarks on the Sachdev-Ye-Kitaev model," Phys. Rev. D 94(10), 106002 (2016); e-print arXiv: 1604.07818 [hep-th].

${ }^{63}$ D. J. Gross and V. Rosenhaus, “A generalization of Sachdev-Ye-Kitaev,” JHEP 1702, 093 (2017); e-print arXiv: 1610.01569 [hep-th].

${ }^{64}$ L. Freidel, "Group field theory: An overview,” Int. J. Theor. Phys. 44, 1769 (2005); e-print arXiv:hep-th/0505016.

${ }^{65}$ D. Oriti (ed.), Approaches to quantum gravity (Cambridge University Press, Cambridge, 2009), pp. 310-331.

${ }^{66}$ D. Oriti, "A quantum field theory of simplicial geometry and the emergence of spacetime," J. Phys. Conf. Ser. 67, 012052 (2007); e-print arXiv:hep-th/0612301.

${ }^{67}$ H. Grosse and R. Wulkenhaar, "Renormalisation of $\phi^{4}$ theory on noncommutative $\mathbb{R}^{4}$ in the matrix base," Commun. Math Phys. 256, 305 (2005); e-print arXiv:hep-th/0401128. 
${ }^{68}$ V. Rivasseau, From Perturbative to Constructive Renormalization, Princeton Series in Physics (Princeton University Press, Princeton, 1991)

${ }^{69}$ G. Gallavotti and F. Nicolò, "Renormalization theory in four-dimensional scalar fields. I." Commun. Math. Phys. 100, 545 (1985).

70 J. P. Ryan, “Tensor models and embedded Riemann surfaces,” Phys. Rev. D 85, 024010 (2012); e-print arXiv:1104.5471 [gr-qc]. 